\title{
Regularity for the CR vector bundle problem II
}

\author{
XiAnghong Gong And Sidney M. Webster
}

\begin{abstract}
We derive a $\mathcal{C}^{k+\frac{1}{2}}$ Hölder estimate for $P \varphi$, where $P$ is either of the two solution operators in Henkin's local homotopy formula for $\bar{\partial}_{b}$ on a strongly pseudoconvex real hypersurface $M$ in $\mathbb{C}^{n}, \varphi$ is a $(0, q)$-form of class $\mathcal{C}^{k}$ on $M$, and $k \geq 0$ is an integer. We also derive a $\mathcal{C}^{a}$ estimate for $P \varphi$, when $\varphi$ is of class $\mathcal{C}^{a}$ and $a \geq 0$ is a real number. These estimates require that $M$ be of class $\mathcal{C}^{k+\frac{5}{2}}$, or $\mathcal{C}^{a+2}$, respectively. The explicit bounds for the constants occurring in these estimates also considerably improve previously known such results.

These estimates are then applied to the integrability problem for CR vector bundles to gain improved regularity. They also constitute a major ingredient in a forthcoming work of the authors on the local $\mathrm{CR}$ embedding problem.
\end{abstract}

Mathematics Subject Classification (2010): 32V05 (primary); 32A26, 32T15 (secondary).

\section{Introduction}

In this paper we will prove the following.

Theorem 1.1. Let $n \geq 4$. Let $M$ be a strongly pseudoconvex real hypersurface in $\mathbb{C}^{n}$ of class $\mathcal{C}^{2}$. Let $\omega$ be an $r \times r$ matrix of continuous $(0,1)$-forms on $M$. Assume that $\bar{\partial}_{M} \omega=\omega \wedge \omega$. Near each point of $M$, there exists a non-singular matrix $A$ of Hölder class $\mathcal{C}^{1 / 2}$ satisfying $\bar{\partial}_{M} A=-A \omega$ and

a) $A \in \mathcal{C}^{a}(M)$, if $\omega \in \mathcal{C}^{a}(M), M \in \mathcal{C}^{a+2}$ and $a>0$ is a real number;

b) $A \in \mathcal{C}^{k+\frac{1}{2}}(M)$, if $\omega \in \mathcal{C}^{k}(M), M \in \mathcal{C}^{k+\frac{5}{2}}$ and $k>0$ is an integer.

If $\omega$ and $A$ are of class $\mathcal{C}^{0}$, the identities $\bar{\partial}_{M} \omega=\omega \wedge \omega$ and $\bar{\partial}_{M} A=-A \omega$ are in the sense of currents; see Section 3. This work is a continuation of [5]. For earlier results see [23] and Ma-Michel [15].

We now describe the above result in terms of an integrability problem for CR vector bundles ([23]). Let $E$ be a complex vector bundle of rank $r$ over $M$ with

Research supported in part by NSF grant DMS-0705426.

Received October 19, 2009; accepted February 18, 2010. 
a connection $D$. For a local frame $e=\left(e_{1}, \ldots, e_{r}\right), D e_{i}=\omega_{i}^{j} e_{j}$, where $\omega=$ $\left(\omega_{i}^{j}\right)$ are connection 1-forms on $M$; by a frame change $\tilde{e}=A e, D \tilde{e}=\tilde{\omega} \tilde{e}$ with $\tilde{\omega}=(d A+A \omega) A^{-1}$. The integrability problem is to find an $A$ such that the new connection forms $\tilde{\omega}=\left(\tilde{\omega}_{i}^{j}\right)$ belong to the ideal $\mathcal{J}(M)$ generated by $(1,0)$-forms on $M$. The integrability condition is that the curvature 2 -forms $d \omega-\omega \wedge \omega$ belong to $\mathcal{J}(M)$.

We want to mention a few ingredients in the proof. The integrability problem is local. As in [5,20], we will use the Henkin local homotopy formula. Let $M \subset$ $\mathbb{C}^{n}$ be a graph over a domain $D \subset \mathbb{R}^{2 n-1}$, given by $y^{n}=\left|z^{\prime}\right|^{2}+\hat{r}\left(z^{\prime}, x^{n}\right)$ with $\hat{r}(0)=\partial \hat{r}(0)=0$, where $z=\left(z^{\prime}, z^{n}\right)$ are standard coordinates. Set $M_{\rho}=M \cap$ $\left\{\left(x^{n}\right)^{2}+y^{n}<\rho^{2}\right\}$ and $D_{\rho}=\pi\left(M_{\rho}\right)$. Suppose that $\bar{D}_{\rho_{0}} \subset D$ and the $\mathcal{C}^{2}$-norm $\|\hat{r}\|_{\rho_{0}, 2}$ of $\hat{r}$ on $D_{\rho_{0}}$ is sufficiently small. For $0<\rho \leq \rho_{0}$ and $n \geq 4$, we have the Henkin homotopy formula

$$
\varphi=\bar{\partial}_{M} P \varphi+Q \bar{\partial}_{M} \varphi
$$

for $(0, q)$-forms $\varphi$ on $\overline{M_{\rho}}$ with $0<q<n-2$. We will prove the following estimates.

(i) Let $a \geq 0$ be a real number. Then

$$
\|P \varphi\|_{(1-\sigma) \rho, a} \leq C_{a} \rho^{-s_{*}} \sigma^{-s}\left(\|\varphi\|_{\rho, a}+\|\hat{r}\|_{\rho, a+2}\|\varphi\|_{\rho, 0}\right) .
$$

(ii) Let $k \geq 0$ be an integer. Then

$$
\begin{gathered}
\|P \varphi\|_{(1-\sigma) \rho, k+\frac{1}{2}} \leq C_{k} \rho^{-s_{*}} \sigma^{-s}\left(\left(1+\|\hat{r}\|_{\rho, \frac{5}{2}}\right)\|\varphi\|_{\rho, k}+\|\hat{r}\|_{\rho, k+\frac{5}{2}}\|\varphi\|_{\rho, 0}\right) ; \\
\|P \varphi\|_{(1-\sigma) \rho, 1 / 2} \leq C \rho^{-1} \sigma^{1-2 n}\|\varphi\|_{\rho, 0}, \quad k=0, \quad q=1 .
\end{gathered}
$$

(See (10.16) for $s, s_{*}$.) We emphasize that the estimates hold for all $0<\rho \leq \rho_{0} \leq 3$ and $0<\sigma<1$. Under the coordinates $\left(z^{\prime}, x^{n}\right)$ of $M,\|\cdot\|_{\rho, a}$ denotes the standard $\mathcal{C}^{a}$-norm on the domain $D_{\rho} \subset \mathbb{R}^{2 n-1}$. The same estimates hold for $Q$; however, the second estimate in (ii), based on a special property of the kernels for $(0,1)$ forms, is not applicable to $Q$ when it operates on $(0, q+1)$ form with $q>0$. See Romero [17] for estimates in Hölder norms for the Heisenberg group case and an example showing necessity of blow-up constants.

The estimate (i) is proved in Proposition 10.1 and (ii) is in Proposition 11.1. The above theorem and two estimates are our main results. With the estimates, we will prove Theorem 1.1 by using a KAM rapid iteration argument as in [5], which avoids the Nash-Moser smoothing techniques.

We now describe some ideas to derive the estimates. The integral operators $P, Q$ are estimated in the same way. Let us focus on $P=P_{0}+P_{1}$, where $P_{1}$ is an integral operator over $\partial M_{\rho}$ and $P_{0}$ is over $M_{\rho}$. Since we need estimates only on shrinking domains, the boundary integral $P_{1}$ can be treated easily. For the interior integral $P_{0}$, via cutoff, the difficulties lie in the case where the $(0, q)$-form 
$\varphi=\sum \varphi_{\bar{J}} d \overline{z^{J}}$ has compact support. We will see that coefficients of $(0, q-1)$-form $P_{0} \varphi$ are sums of $\mathcal{K} f(z)=\int_{M_{\rho}} f(\zeta) k(\zeta, z) d V(\zeta)$, where $f(\zeta)=\varphi_{\bar{J}}(\zeta) U_{\bar{J}}(\zeta)$ and $U_{\bar{J}}$ depend on derivatives of $\left|\zeta^{\prime}\right|^{2}+\hat{r}\left(\zeta^{\prime}, \xi^{n}\right)$ of order at most two, and

$$
k(\zeta, z)=\frac{r_{\zeta^{j}}-r_{z^{j}}}{\left(r_{\zeta} \cdot(\zeta-z)\right)^{a}\left(r_{z} \cdot(\zeta-z)\right)^{b}}, \quad a=n-q, b=q
$$

for $r(z)=-y^{n}+\left|z^{\prime}\right|^{2}+\hat{r}\left(z^{\prime}, x^{n}\right)$; see Section 3 for details.

To deal with the singularity of the kernel along $\zeta=z$, we fix $z \in M$ and apply the approximate Heisenberg transformation $\zeta \rightarrow \zeta_{*}$ defined by

$$
\psi_{z}: \zeta_{*}^{\prime}=\zeta^{\prime}-z^{\prime}, \quad \zeta_{*}^{n}=-2 i r_{z} \cdot(\zeta-z) .
$$

We will show that $\zeta_{*}^{\prime}, \xi_{*}^{n}=2 \operatorname{Im}\left(r_{z} \cdot(\zeta-z)\right)$ form coordinates of $\psi_{z}(M)$, and under this coordinate system the integral becomes

$$
\mathcal{K} f(z)=\int_{\pi\left(\psi_{z}\left(M_{\rho}\right)\right)} f\left(\psi_{z}^{-1}\left(\zeta_{*}\right)\right) k_{*}\left(\zeta_{*}^{\prime}, \xi_{*}^{n}, z^{\prime}, x^{n}\right) d V\left(\zeta_{*}^{\prime}, \xi_{*}^{n}\right) .
$$

Here

$$
\begin{gathered}
k_{*}\left(\zeta_{*}^{\prime}, \xi_{*}^{n}, z^{\prime}, x^{n}\right)=\sum_{|I|=1} E_{I}\left(\zeta_{*}^{\prime}, \xi_{*}^{n}, z^{\prime}, x^{n}\right) \hat{k}_{a b}^{I}\left(\zeta_{*}^{\prime}, \xi_{*}^{n}\right), \\
\hat{k}_{a b}^{I}\left(\zeta_{*}^{\prime}, \xi_{*}^{n}\right)=\frac{\left(\zeta_{*}^{\prime}, \overline{\zeta_{*}^{\prime}}, \xi_{*}^{n}\right)^{I}}{\left(\left|\zeta_{*}^{\prime}\right|^{2}+i \xi_{*}^{n}\right)^{a}\left(\left|\zeta_{*}^{\prime}\right|^{2}-i \xi_{*}^{n}\right)^{b}}, \quad a+b=n,
\end{gathered}
$$

where $I=\left(i_{1}, \ldots, i_{2 n-1}\right)$ and we use standard multi-index notation. The coefficients $E_{I}\left(\zeta_{*}^{\prime}, \xi_{*}^{n}, \cdot\right)$ are of class $\mathcal{C}^{a}$ if $\left(\zeta_{*}^{\prime}, \xi_{*}^{n}\right)(\neq 0)$ is fixed and $\hat{r} \in \mathcal{C}^{a+2}$. Since $f$ has compact support, one can take derivatives of $\mathcal{K} f$ directly onto $f$ and onto $E_{I}$ without disturbing the kernels $\hat{k}_{a b}^{I}$. The transformation $\psi_{z}$ has been used by other people. See Bruna-Burgués [1] and Ma-Michel [13]. To obtain estimate (ii), we need to return to the original coordinates after differentiation. This will give us another formula for the derivatives of $\mathcal{K} f$, which allows us to reduce the $\mathcal{C}^{k+\frac{1}{2}}$-estimates to the Hölder $\frac{1}{2}$-estimate for new kernels of the same type.

We would like to mention some methods to derive the fundamental $\frac{1}{2}$-estimate. Kerzman [11] obtained Hölder $\alpha$-estimates for all $\alpha<\frac{1}{2}$ for $\bar{\partial}$-solutions, by estimating a Cauchy-Fantappiè form. Folland-Stein [3] used non-isotropic balls and piecewise smooth curves in complex tangential directions to obtain estimates in their spaces on the real hyperquadric. Henkin-Romanov [8] obtained the $\frac{1}{2}$-estimate for $\bar{\partial}$-equations on strongly pseudoconvex domains via a type of Hardy-Littlewood lemma (see also Henkin [7] for $\bar{\partial}_{b}$ on strictly convex boundaries). Our estimate, like the classical Hölder estimate for the Newtonian potential, is still based on a decomposition of domain. However, we delete a cylinder about the pole, instead of a (non-isotropic) ball. The radius of the cylinder is optimized for the $\frac{1}{2}$-exponent 
and is yet so large that, when estimating the Hölder $\frac{1}{2}$-ratio at two points, we can ignore their non-isotropic distance and connect them with a line segment.

In the 5 dimensional case there is an extra term added to the right-hand side of (1.1). As of this writing, it remains unclear whether such a more general homotopy formula can be used. See [21] and Nagel-Rosay [16].

The $\frac{1}{2}$-estimate for a solution to $\bar{\partial}$-equations for $(0,1)$-forms on strictly pseudoconvex domains with $\mathcal{C}^{2}$ boundary is obtained by Henkin-Romanov [8]. For $\mathcal{C}^{k+\frac{1}{2}}$-estimates for solutions of $\bar{\partial}$-equations of degree $(0,1)$ on strictly pseudoconvex domains with $\mathcal{C}^{m}$ boundary ( $\left.m \geq k+4\right)$, see Siu [19]; for $\bar{\partial}$-equations in higher degree, see Lieb-Range [12]. The $\mathcal{C}^{0}$-estimate for a solution to $\bar{\partial}_{b}$-equations on $M_{\rho}$, without shrinking $M_{\rho}$, is given by Henkin [7]. For $\mathcal{C}^{k}$-estimates for the homotopy formula for $\bar{\partial}_{b}$ operator on shrinking domains, see [21]. Michel-Ma [13] also obtain $\mathcal{C}^{k}$-estimates for a modified homotopy formula without shrinking domains, by introducing an extra derivative via $\bar{\partial}_{b}$.

We want to mention that in estimating $(i)$ and (ii) we need some Hölder inequalities. For the convenience of the reader, we present these inequalities in Appendix A, following the formulation and proofs of Hörmander [9].

The estimates $(i)$ and (ii) will be used to improve regularity in the local CR embedding problem in [6]. To limit the scope of this paper, we leave the estimates in Folland-Stein spaces for future work.

\section{Notation and counting derivatives}

To simplify notation, set $z^{\prime}=\left(z^{1}, \ldots, z^{n-1}\right), z=\left(z^{\prime}, z^{n}\right)$, and

$$
x=\left(\operatorname{Re} z, \operatorname{Im} z^{\prime}\right)=\pi(z) .
$$

Analogously, $\xi=\left(\operatorname{Re} \zeta, \operatorname{Im} \zeta^{\prime}\right)$. Denote by $|\cdot|$ the Euclidean norms on $\mathbb{C}^{n-1}, \mathbb{C}^{n-1} \times$ $\mathbb{R}=\mathbb{R}^{2 n-1}$ and $\mathbb{C}^{n}$. Our real hypersurface $M \subset \mathbb{C}^{n}$ is always a graph over a domain in $\mathbb{C}^{n-1} \times \mathbb{R}$. Let $M_{\rho}=M \cap\left\{\left(x^{n}\right)^{2}+y^{n}<\rho^{2}\right\}$ and $D_{\rho}=\pi\left(M_{\rho}\right)$. For the real hyperquadric $M: y^{n}=\left|z^{\prime}\right|^{2}, \pi\left(M_{\rho}\right)$ is exactly the ball $B_{\rho}=\left\{x \in \mathbb{R}^{2 n-1}:|x|<\right.$ $\rho$ \}. On $\mathbb{R}^{2 n-1}$, we will use the volume-form $d V=d \xi^{1} \wedge d \eta^{1} \wedge \cdots \wedge d \eta^{n-1} \wedge d \xi^{n}$. On $\partial D_{\rho}$, we will need $(2 n-2)$-forms $d V^{s}=d \xi^{1} \wedge \cdots \wedge d \hat{\xi}^{s} \wedge \cdots \wedge d \xi^{2 n-1}$.

Let $k \geq 0$ be an integer. Denote by $\partial^{I} u$ a derivative of $u$ of order $|I|$, where $I$ is a standard multi-index. Let $\partial^{k} u$ denote the set of the $k$-th order derivatives of $u$. For a function $u$ on $D \subset \mathbb{R}^{2 n-1}$, define

$$
\begin{gathered}
\left\|\partial^{k} u\right\|_{D, 0}=\sup _{x \in D,|I|=k}\left|\partial^{I} u(x)\right|, \quad\|u\|_{D, k}=\max _{0 \leq j \leq k}\left\|\partial^{j} u\right\|_{D, 0}, \\
|u|_{D, \alpha}=\sup _{x, y \in D} \frac{|u(x)-u(y)|}{|x-y|^{\alpha}}, \quad 0<\alpha<1, \\
\|u\|_{D, k+\alpha}=\max \left\{\|u\|_{D, k},\left|\partial^{I} u\right|_{D, \alpha}:|I|=k\right\}, \quad 0<\alpha<1 .
\end{gathered}
$$


If $A=\left(a_{i}^{j}\right)$ is a matrix of $\mathcal{C}^{k}$ functions on $D$, we define $\|A\|_{D, k}=\max _{i, j}\left\{\left\|a_{i}^{j}\right\|_{D, k}\right\}$. Define $\|A\|_{D, k+\alpha}$ analogously.

To avoid confusion and in the essence of estimates $(i)$-(ii) in the introduction, the $\mathcal{C}^{a}$-norm of a function on $M_{\rho}$ is its $\mathcal{C}^{a}$-norm on $D_{\rho} \subset \mathbb{R}^{2 n-1}$, defined above. The $\mathcal{C}^{a}\left(M_{\rho}\right)$ norm of a $(0, q)$-form $\varphi=\sum \varphi_{\bar{I}} d{\overline{z^{\prime}}}^{I}$ is the maximum of $\mathcal{C}^{a}$-norms of $\varphi_{\bar{I}}$. The $\mathcal{C}^{a}$-norm on $M_{\rho}$ will be denoted by $\|\cdot\|_{\mathcal{C}^{a}\left(M_{\rho}\right)}=\|\cdot\|_{D_{\rho}, a}$, or simply by $\|\cdot\|_{\rho, a}$ when there is no confusion about $M$.

Throughout the paper, $C_{k}$ denotes a constant dependent of $k$ and this dependence will not be expressed sometimes. Constants, such as $C, C_{1}, C_{k}$, might have different values when they reoccur. All constants are independent of $M, \hat{r}, \rho, \rho_{0}$.

Let $M$ be defined by

$$
r(z) \stackrel{\text { def }}{=}-y^{n}+\left|z^{\prime}\right|^{2}+\hat{r}(x)=0, \quad x \in D,
$$

where $\hat{r} \in \mathcal{C}^{2}(D)$. Throughout the paper, we make the basic assumption

$$
\overline{D_{\rho_{0}}} \subset D, \quad \hat{r}(0)=\hat{r}_{z}(0)=0, \quad \epsilon=\|\hat{r}\|_{\rho_{0}, 2}<C_{0}^{-1} .
$$

We emphasize that $C_{0}$ is a large constant to be adjusted several times. However, it will not depend on any quantity other than $n$.

Counting derivatives. We need to count derivatives efficiently and use the count to estimate norms. Such a counting scheme is essentially in [9] and we specialize it for two reasons. First, the homotopy formula involves two extra derivatives of the defining function $r$ of $M$; second, for each consecutive $x$-derivative on $\varphi_{\bar{J}} \circ \psi_{z}^{-1}$, the $x$-derivative which falls on $\varphi_{\bar{J}}$ yields an extra factor $\partial^{2} r(z)$ via the chain rule and $\psi_{z}(\zeta)=\left(\zeta^{\prime}-z^{\prime},-i 2 r_{z} \cdot(\zeta-z)\right)$. We illustrate below how to use the scheme to cope with the two extra derivatives on $r$ and the consecutive derivatives.

Recall that for $l \geq 1, \partial^{l} r(z)=\partial^{l} r(x)$ is the set of the $l$-th order derivatives of $r$. Define

$$
\begin{aligned}
& \partial_{*}^{1} r(\xi, x)=p\left(\xi, x,\left(1+\hat{r}_{x^{n}} \hat{r}_{\xi^{n}}\right)^{-1}, r_{z^{n}}^{-1}, \partial \hat{r}(\xi), \partial \hat{r}(x)\right), \\
& \partial_{*}^{2} r(\xi, x)=p\left(\xi, x,\left(1+\hat{r}_{x^{n}} \hat{r}_{\xi^{n}}\right)^{-1}, r_{z^{n}}^{-1}, \partial \hat{r}(\xi), \partial^{2} \hat{r}(\xi), \partial \hat{r}(x), \partial^{2} \hat{r}(x)\right) .
\end{aligned}
$$

Here and in what follows, $p$ is a polynomial with constant coefficients. Its coefficients and degree are bounded in absolute values by a constant depending only on fixed quantities, say $k, n$. Also, $p$ might be different when it reoccurs. In general, define

$$
\partial_{*}^{2+k} r(\xi, x)=\sum \partial_{*}^{2} r(\xi, x) \partial^{I_{1}} r(\xi) \cdots \partial^{I_{j}} r(\xi) \partial^{J_{1}} r(x) \cdots \partial^{J_{l}} r(x),
$$

where the sum is over finitely many multi-indices $I_{i}, J_{i}$ satisfying

$$
\sum_{i=1}^{j}\left(\left|I_{i}\right|-2\right)+\sum_{i=1}^{l}\left(\left|J_{i}\right|-2\right) \leq k, \quad\left|I_{i}\right| \geq 2, \quad\left|J_{i}\right| \geq 2 .
$$


We will write $\partial_{*}^{2+k} r(\xi, x)=\partial_{*}^{2+k} r(x)$ when it depends only on $x$. With this abbreviation, we have simple relations

$$
\partial_{*}^{2+k} r \partial_{*}^{2+j} r=\partial_{*}^{2+k+j} r, \quad \partial^{J} \partial_{*}^{2+k} r=\partial_{*}^{2+k+|J|} r .
$$

From [9, Corollary A.6] (see Proposition A.5 in Appendix A), we know that with $D_{\rho}^{2}=D_{\rho} \times D_{\rho}$,

$$
\prod_{j=1}^{m}\left\|f_{j}\right\|_{D_{\rho}^{2}, k_{j}+b_{j}} \leq C_{|a|+|c|+m} \rho^{-b_{1}-\cdots-b_{m}}\left(\prod_{j=1}^{m}\left\|f_{j}\right\|_{D_{\rho}^{2}, k_{j}+a_{j}}+\prod_{j=1}^{m}\left\|f_{j}\right\|_{D_{\rho}^{2}, k_{j}+c_{j}}\right)
$$

for any non-negative integers $k_{j}$ and non-negative real numbers $a_{j}, c_{j}$ such that $\left(b_{1}, \ldots, b_{m}\right)$ is in the convex hull of $\left(a_{1}, \ldots, a_{m}\right),\left(c_{1}, \ldots, c_{m}\right)$. With the above abbreviation, basic assumption (2.2), and $0<\rho \leq \rho_{0} \leq 3$, one obtains

$$
\left\|\partial_{*}^{2+k} r\right\|_{\rho, a} \leq C_{a+k} \rho^{-a-k}\|r\|_{\rho, 2+k+a}, \quad\|r\|_{\rho, 2+k+a} \stackrel{\text { def }}{=} 1+\|\hat{r}\|_{\rho, 2+k+a}
$$

for all real numbers $a \geq 0$ and integers $k \geq 0$.

We will also need a chain rule. Recall that $\psi_{z}: \zeta_{*}^{\prime}=\zeta^{\prime}-z^{\prime}, \zeta_{*}^{n}=-2 i r_{z} \cdot(\zeta-z)$ and define

$$
\Psi(\xi, x)=\left(\pi \psi_{z}(\zeta), x\right), \quad z=\left.\pi\right|_{M} ^{-1}(x), \quad \zeta=\left.\pi\right|_{M} ^{-1}(\xi) .
$$

Let $0<\rho \leq \rho_{0} \leq 3$. We will show that $B_{\rho / 2} \subset D_{\rho} \subset B_{2 \rho}$ and

$$
W_{\rho}=\Psi\left(D_{\rho} \times D_{\rho}\right) \subset B_{9 \rho} \times D_{\rho} .
$$

(See Lemmas 5.1-5.2.) The Jacobean matrix of $\Psi$ depends only on derivatives of $r$ of order $\leq 2$ and has determinant $1+\hat{r}_{x^{n}} \hat{r}_{\xi^{n}}$ (by (6.2)). Then the chain rule takes simple forms. Let $\left(\Psi^{-1}\right)^{j}$ be the $j$-th component of $\Psi^{-1}$. Then

$$
\begin{gathered}
\partial^{I}\left\{\left(\Psi^{-1}\right)^{j}\right\}=\partial_{*}^{2} r \circ \Psi^{-1}, \quad|I|=1 ; \\
\partial^{K}\left(f \circ \Psi^{-1}\right)=\sum_{|L| \leq|K|}\left(\partial^{L} f \partial_{*}^{2+|K|-|L|} r\right) \circ \Psi^{-1} .
\end{gathered}
$$

We will show that the Lipschitz constant of $\Psi^{-1}$ on $W_{\rho}$ is bounded by $C$ (see Lemma 5.2). Then taking Hölder ratio in (2.6) with $k=[a]$ gives us

$$
\left\|f \circ \Psi^{-1}\right\|_{W_{\rho}, a} \leq C_{k} \rho^{-a}\left(\|f\|_{D_{\rho} \times D_{\rho}, a}+\|f\|_{D_{\rho} \times D_{\rho}, 0}\|r\|_{\rho, 2+a}\right) .
$$

Note that the above mentioned $\varphi_{\bar{J}} \circ \psi_{z}^{-1}$ is a special case.

The above counting scheme via (2.4)-(2.7) and its variants will be used systematically. 


\section{The Henkin homotopy formula}

In this section we recall the homotopy formula by following the formulation in [21]. We discuss the formula for differential forms of low regularity.

Let $M \subset \mathbb{C}^{n}: r=0$ be given by (2.1)-(2.2). We first recall a representative $\bar{\partial}_{M}$ for the $\bar{\partial}_{b}$-operator. By definition, a $(p, q)$-form $\varphi$ of class $\mathcal{C}^{a}$ on $M$ is the restriction of some $(p, q)$-form $\tilde{\varphi}$ of class $\mathcal{C}^{a}$ in a neighborhood of $M$. If $a \geq 1$, we define $\bar{\partial}_{b} \varphi$ to be the restriction of $\bar{\partial} \tilde{\varphi}$ to $M$. Notice that on $M, \theta=-2 i \partial r=\bar{\theta}$ and that $\bar{\partial}_{b} \varphi$ is well-defined modulo $\bar{\partial} r$ when $0 \leq p<n$, and it is actually well-defined when $p=n$. By a tangential $(0, q)$-form $\varphi$, we mean a form $\varphi=\sum_{|I|=q} \varphi_{\bar{I}} d \overline{z^{\prime}} I$ with $d \overline{z^{\prime}}=\left(d \overline{z^{1}}, \ldots, d \overline{z^{n-1}}\right)$. A continuous $(0, q)$-form $\varphi$ can be written uniquely as $\varphi^{\prime}+\varphi^{\prime \prime} \wedge \bar{\theta}$ for some tangential forms $\varphi^{\prime}, \varphi^{\prime \prime}$. Define

$$
\overline{X_{\alpha}}=\partial_{z^{\alpha}}-\frac{r_{z^{\alpha}}}{r_{z^{n}}} \partial_{z^{n}}, \quad \bar{\partial}_{M} \varphi=\bar{\partial}_{M} \varphi^{\prime}=\sum_{|I|=q} \sum_{1 \leq \alpha<n} \overline{X_{\alpha}} \varphi_{\bar{I}}^{\prime} d \overline{z^{\alpha}} \wedge d \overline{z^{\prime}} I .
$$

Then a straightforward computation shows that

$$
\bar{\partial}_{b} \varphi=\bar{\partial}_{M} \varphi \bmod \theta .
$$

Each $(n, q)$-form on $M$ can be written as $\varphi^{\prime \prime} \wedge d z^{1} \wedge \cdots \wedge d z^{n-1} \wedge \theta$ where $\varphi^{\prime \prime}$ is tangential. If $\varphi^{\prime \prime} \in \mathcal{C}^{1}$, then

$$
\bar{\partial}_{b}\left(\varphi^{\prime \prime} \wedge d z^{1} \wedge \cdots \wedge d z^{n-1} \wedge \theta\right)=\bar{\partial}_{M} \varphi^{\prime \prime} \wedge d z^{1} \wedge \cdots \wedge d z^{n-1} \wedge \theta .
$$

Let $\varphi$ be a continuous $(0, q)$-form on a domain $\mathcal{U} \subset M$, and $\phi$ be a continuous $(0, q-1)$-form. Suppose that $q \geq 1$. We say that $\bar{\partial}_{b} \phi=\varphi \bmod \theta$ holds on $\mathcal{U}$ as currents, if $\int_{M} \phi \wedge \bar{\partial}_{b} \psi=(-1)^{q} \int_{M} \varphi \wedge \psi$ for all $\mathcal{C}^{1}$-smooth $(n, n-q-1)$-forms $\psi$ with compact support in $\mathcal{U}$.

Write $r_{z}=r_{z}(z)$ and $r_{\zeta}=r_{\zeta}(\zeta)$. Set $N_{0}(\zeta, z)=r_{\zeta} \cdot(\zeta-z), S_{0}(\zeta, z)=$ $r_{z} \cdot(\zeta-z)$ and

$$
\begin{gathered}
\Omega_{0, q-1}^{+-}(\zeta, z)=\frac{\partial_{\zeta} r \wedge\left(r_{z} \cdot d \zeta\right) \wedge\left(\partial_{\bar{\zeta}} \partial_{\zeta} r\right)^{n-1-q} \wedge\left(\partial_{\bar{z}} r_{z} \wedge d \zeta\right)^{q-1}}{N_{0}^{n-q}(\zeta, z) S_{0}^{q}(\zeta, z)} \\
\Omega_{0, q-1}^{0+-}(\zeta, z)=\frac{d \zeta^{n} \wedge \partial_{\zeta} r \wedge\left(r_{z} \cdot d \zeta\right) \wedge\left(\partial_{\bar{\zeta}} \partial_{\zeta} r\right)^{n-2-q} \wedge\left(\partial_{z} r_{z} \wedge d \zeta\right)^{q-1}}{\left(\zeta^{n}-z^{n}\right) N_{0}^{n-q-1}(\zeta, z) S_{0}^{q}(\zeta, z)}
\end{gathered}
$$

Note that $\theta(\zeta)$ annihilates $\Omega_{0, q-1}^{+-}(\zeta, z)$ and $\Omega_{0, q-1}^{0+-}(\zeta, z)$. Let $n \geq 4$ and $0<q<$ $n-2$. For a tangential $(0, q)$-form $\varphi$ on $\overline{M_{\rho}}$, we have the homotopy formula

$$
\varphi=\bar{\partial}_{M}\left(P_{0}^{\prime}+P_{1}^{\prime}\right) \varphi+\left(Q_{0}^{\prime}+Q_{1}^{\prime}\right) \bar{\partial}_{M} \varphi, \quad z \in M_{\rho} .
$$


Here $P_{0}^{\prime}, P_{1}^{\prime}, Q_{0}^{\prime}, Q_{1}^{\prime}$ are tangential parts of

$$
\begin{array}{ll}
P_{0} \varphi(z)=c_{1} \int_{M_{\rho}} \varphi \wedge \Omega_{0, q-1}^{+-}(\zeta, z), & P_{1} \varphi(z)=c_{2} \int_{\partial M_{\rho}} \varphi \wedge \Omega_{0, q-1}^{0+-}(\zeta, z), \\
Q_{0} \psi(z)=c_{3} \int_{M_{\rho}} \psi \wedge \Omega_{0, q}^{+-}(\zeta, z), & Q_{1} \psi(z)=c_{4} \int_{\partial M_{\rho}} \psi \wedge \Omega_{0, q}^{0+-}(\zeta, z) .
\end{array}
$$

From now on, all $(0, q)$-forms $\varphi$ on $M$ are tangential. By $\|\varphi\|_{\rho, a}$, we mean the norm $\left\|\varphi^{\prime}\right\|_{\rho, a}$ as defined in Section 2, where $\varphi^{\prime}=\varphi \bmod \theta$ and $\varphi^{\prime}$ is tangential. By $\|P \varphi\|_{\rho, a}$ as used in the introduction, where $P$ is either of solution operators in the homotopy formula, we mean $\left\|P^{\prime} \varphi\right\|_{\rho, a}$. By an abuse of notation, $\varphi$ stands for forms on $M_{\rho}$ and $D_{\rho}=\pi\left(M_{\rho}\right)$.

Next, we describe kernels of $P_{j}^{\prime}, Q_{j}^{\prime}$ on domain $D_{\rho}$ via coordinates $x$. We have

$$
\begin{gathered}
r_{\zeta} \cdot d \zeta \wedge r_{z} \cdot d \zeta=\sum_{1 \leq j, l \leq n} r_{\zeta^{l}}\left(r_{\zeta^{j}}-r_{z^{j}}\right) d \zeta^{j} \wedge d \zeta^{l} \\
r_{\zeta} \cdot d \zeta \wedge r_{z} \cdot d \zeta \wedge d \zeta^{n}=\sum_{1 \leq \alpha, \beta<n} r_{\zeta^{\beta}}\left(r_{\zeta^{\alpha}}-r_{z^{\alpha}}\right) d \zeta^{\alpha} \wedge d \zeta^{\beta} \wedge d \zeta^{n} .
\end{gathered}
$$

Assume now that $\zeta, z \in M_{\rho}$. We compute $d \overline{\zeta^{n}}$ and $d \overline{z^{n}}$ in different ways. We keep the latter a $(0,1)$-form and find its tangential part. We have

$$
\begin{gathered}
d \overline{z^{n}}=-\frac{r_{\overline{z^{\prime}}}}{r_{\overline{z^{n}}}} \cdot d \overline{z^{\prime}} \bmod \theta(z), \\
d \zeta^{n}=\left(1+i \hat{r}_{\xi^{n}}\right) d \xi^{n}+i 2 \operatorname{Re}\left\{r_{\zeta^{\prime}} \cdot d \zeta^{\prime}\right\}=2 i r_{\xi^{n}} d \xi^{n}+i 2 \operatorname{Re}\left\{r_{\zeta^{\prime}} \cdot d \zeta^{\prime}\right\} .
\end{gathered}
$$

Note that in (3.9), we have used $r(\zeta)=-\eta^{n}+\left|\zeta^{\prime}\right|^{2}+\hat{r}(\xi)=0$. In (3.6)-(3.7) and $\bar{\partial}_{z} r_{z} \wedge d \zeta$, we use (3.8)-(3.9) to rewrite $d \overline{z^{n}}$ and $d \zeta^{n}$, respectively. In $\partial_{\bar{\zeta}} \partial_{\zeta} r$, we use (3.9) to rewrite $d \zeta^{n}, d \overline{\zeta^{n}}$. From (3.2)-(3.3), we obtain on $M_{\rho}$

$$
\begin{aligned}
& P_{0}^{\prime} \varphi(x)=\sum_{|I|=q-1} \sum_{|J|=q} \sum_{1 \leq j \leq n} d{\overline{z^{\prime}}}^{I} \int_{D_{\rho}} A_{\bar{I}}^{j \bar{J}}(\xi, x) \frac{\varphi_{\bar{J}}(\xi)\left(r_{\zeta^{j}}-r_{z^{j}}\right)}{\left(N_{0}^{n-q} S_{0}^{q}\right)(\zeta, z)} d V(\xi), \\
& P_{1}^{\prime} \varphi(x)=\sum_{|I|=q-1} \sum_{|J|=q} \sum_{\alpha, \beta=1}^{n-1} \sum_{s=1}^{2 n-1} d{\overline{z^{\prime}}}^{I} \int_{\partial D_{\rho}} \frac{B_{\bar{I} s}^{\alpha \beta \bar{J}}(\xi, x) \varphi_{\bar{J}}(\xi)\left(r_{\zeta^{\alpha}}-r_{z^{\alpha}}\right) r_{\zeta^{\beta}}}{\left(\zeta^{n}-z^{n}\right)\left(N_{0}^{n-q-1} S_{0}^{q}\right)(\zeta, z)} d V^{s}(\xi) .
\end{aligned}
$$

Here $A_{\bar{I}}^{j \bar{J}}$ and $B_{\bar{I} s}^{\alpha \beta \bar{J}}$ are polynomials in $\left(r_{\zeta}, r_{\bar{\zeta}}, r_{\zeta \bar{\zeta}}, r_{\bar{z}^{\prime}}, 1 / r_{\bar{z}^{n}}, r_{z \bar{z}}\right)$. We make a remark for the case $q=1$. In this case we need to remove $\sum_{|I|=0}$ in (3.10)-(3.11). Also, $A^{j \bar{J}} \stackrel{\text { def }}{=} A_{\bar{I}}^{j \bar{J}}$ and $B_{s}^{\alpha \beta \bar{J}} \stackrel{\text { def }}{=} B_{\bar{I} s}^{\alpha \beta \bar{J}}$ are independent of $z$. This observation will play a role in the $\frac{1}{2}$-estimate of $P^{\prime} \varphi$ when $\varphi$ is a $(0,1)$-form.

See also Chen-Shaw [2] for homotopy formulae. In this paper we replace the strict convexity of defining function $r$ in [2] by the condition (2.2) with $0<\rho_{0} \leq 3$; 
see Appendix B for details. We remark that the homotopy formula (3.4) holds as currents, when $\varphi$ and $\bar{\partial}_{b} \varphi$ on $M_{\rho}$ admit continuous extensions to $\bar{M}_{\rho}$. See Appendix B for a proof by using the Friedrichs approximation theorem. Henkin [7] formulated $\bar{\partial}_{b}$ in the sense of currents. Shaw [18] also used the Friedrichs approximation for $\bar{\partial}_{b}$-solutions.

\section{Kernels and the approximate Heisenberg transformation}

In this section, we will describe briefly the new kernels when the approximate Heisenberg transformation is applied. The contents of next few sections are indicated at the end of this section.

Recall that in (3.10) functions $A_{\bar{I}}^{j \bar{J}}$ have the form $\partial_{*}^{2} r$. Hence coefficients of $P_{0}^{\prime} \varphi$ are sums over $|J|=q$ and $1 \leq j \leq n$ of $\mathcal{K} \varphi_{\bar{J}}(x)=\int_{D_{\rho}} \varphi_{\bar{J}}(\xi) k_{a b}^{j}(\xi, x) d V(\xi)$. Here $\zeta, z \in M$ and

$$
k(\xi, x)=k_{a b}^{j}(\xi, x)=\frac{\partial_{*}^{2} r(\xi, x)\left(r_{\zeta^{j}}-r_{z^{j}}\right)}{\left(r_{\zeta} \cdot(\zeta-z)\right)^{a}\left(r_{z} \cdot(\zeta-z)\right)^{b}}, \quad a=n-q, b=q .
$$

To understand the kernel, let us compute its denominator. Set $\hat{r}(z)=\hat{r}(x)$. On $M \times M$ we have

$$
\begin{aligned}
r_{z} \cdot(\zeta-z)= & \overline{z^{\prime}} \cdot\left(\zeta^{\prime}-z^{\prime}\right)+\frac{i}{2}\left(\zeta^{n}-z^{n}\right)+\hat{r}_{z} \cdot(\zeta-z) \\
= & \overline{z^{\prime}} \cdot\left(\zeta^{\prime}-z^{\prime}\right)-\frac{1}{2}\left(\left|\zeta^{\prime}\right|^{2}-\left|z^{\prime}\right|^{2}\right)+\frac{i}{2}\left(\xi^{n}-x^{n}\right) \\
& -\frac{1}{2}(\hat{r}(\zeta)-\hat{r}(z))+\hat{r}_{z} \cdot(\zeta-z) \\
= & i \operatorname{Im}\left(r_{z} \cdot(\zeta-z)\right)-\frac{1}{2}\left|\zeta^{\prime}-z^{\prime}\right|^{2} \\
& -\frac{1}{2}(\hat{r}(\zeta)-\hat{r}(z))+\operatorname{Re}\left(\hat{r}_{z} \cdot(\zeta-z)\right) .
\end{aligned}
$$

Also

$$
\begin{aligned}
r_{\zeta} \cdot(\zeta-z)= & r_{z} \cdot(\zeta-z)+\left(r_{\zeta}-r_{z}\right) \cdot(\zeta-z) \\
= & i \operatorname{Im}\left(r_{z} \cdot(\zeta-z)\right)+\frac{1}{2}\left|\zeta^{\prime}-z^{\prime}\right|^{2}+\left(\hat{r}_{\zeta}-\hat{r}_{z}\right) \cdot(\zeta-z) \\
& -\frac{1}{2}\left\{\hat{r}(\zeta)-\hat{r}(z)-2 \operatorname{Re}\left(\hat{r}_{z} \cdot(\zeta-z)\right)\right\} .
\end{aligned}
$$

The first two terms in $r_{z} \cdot(\zeta-z)$ and $r_{\zeta} \cdot(\zeta-z)$ can be simplified simultaneously. Define

$$
N(\zeta, z) \stackrel{\text { def }}{=}\left|\zeta^{\prime}-z^{\prime}\right|^{2}+2 i \operatorname{Im}\left(r_{z} \cdot(\zeta-z)\right)
$$


Then we arrive at the basic relations

$$
-2 r_{z} \cdot(\zeta-z)=\overline{N(\zeta, z)}+A(\zeta, z), \quad 2 r_{\zeta} \cdot(\zeta-z)=N(\zeta, z)+B(\zeta, z)
$$

with

$$
A(\zeta, z)=\hat{r}(\zeta)-\hat{r}(z)-2 \operatorname{Re}\left(\hat{r}_{z} \cdot(\zeta-z)\right), \quad B(\zeta, z)=2\left(\hat{r}_{\zeta}-\hat{r}_{z}\right) \cdot(\zeta-z)-A(\zeta, z)
$$

By the condition (2.2) on $\hat{r}$, we will show that $D_{\rho_{0}}$ is convex and hence

$$
|A(\zeta, z)| \leq C \epsilon|\zeta-z|^{2}, \quad|B(\zeta, z)| \leq C \epsilon|\zeta-z|^{2}, \quad \zeta, z \in M_{\rho_{0}}
$$

We will show that $|N(\zeta, z)| \geq C^{-1}|\zeta-z|^{2}$ on $M_{\rho_{0}} \times M_{\rho_{0}}$. Consequently, the kernel of $P_{0}^{\prime} \varphi$ is factored as

$$
\begin{gathered}
k(\xi, x)=T_{1}(\xi, x)^{-a} T_{2}(\xi, x)^{-b} \frac{\partial_{*}^{2} r(\xi, x)\left(r_{\zeta^{j}}-r_{z^{j}}\right)}{N^{a}(\zeta, z) \overline{N^{b}(\zeta, z)}} \\
T_{1}(\xi, x)=1+N^{-1}(\zeta, z) B(\zeta, z), \quad T_{2}(\xi, x)=1+\overline{N^{-1}(\zeta, z)} A(\zeta, z)
\end{gathered}
$$

for $\zeta, z \in M_{\rho_{0}}$. Moreover, $\min \left\{\left|T_{1}(\xi, x)\right|,\left|T_{2}(\xi, x)\right|\right\} \geq 1 / 4$ when $(\xi, x)$ is on $D_{\rho_{0}} \times D_{\rho_{0}}$ and off its diagonal. Now, identity (4.1) suggests the following approximate Heisenberg transformation

$$
\psi_{z}: \zeta_{*}^{\prime}=\zeta^{\prime}-z^{\prime}, \quad \zeta_{*}^{n}=-2 i r_{z} \cdot(\zeta-z)
$$

We will show that for fixed $z \in M_{\rho_{0}}, \zeta_{*}^{\prime}=\zeta^{\prime}-z^{\prime}, \xi_{*}^{n}=2 \operatorname{Im}\left(r_{z} \cdot(\zeta-z)\right)$ indeed form coordinates of $M_{\rho_{0}}$. Thus, with $\zeta, z \in M_{\rho_{0}}$

$$
\begin{gathered}
N(\zeta, z)=\left|\zeta_{*}^{\prime}\right|^{2}+i \xi_{*}^{n} \stackrel{\text { def }}{=} N_{*}\left(\xi_{*}\right), \\
k(\xi, x)=\sum_{|I|=1} E_{I}\left(\xi_{*}, x\right) \xi_{*}^{I} N_{*}\left(\xi_{*}\right)^{-a} \overline{N_{*}^{-b}\left(\xi_{*}\right)}, \quad a+b=n .
\end{gathered}
$$

When $\varphi$ has compact support, the decomposition for $k(\xi, x)$ allows us to take $x$ derivatives of $K \varphi \bar{J}(x)$ directly onto coefficients $E_{I}\left(\xi_{*}, x\right)$ and onto $\varphi_{\bar{J}}\left(\psi_{z}^{-1}\left(\xi_{*}\right)\right)$, without destroying the integrability of the kernels.

We now describe the contents of next few sections.

We will carry out the details of this section in Sections 5, 6, and 8. In Section 5 we will also study how domains $B_{\rho}, D_{\rho}=\pi\left(M_{\rho}\right)$ and $\pi \psi_{z}\left(M_{\rho}\right)$ are nested. We will need Hölder inequalities in Appendix A for domains $D_{\rho}$. Therefore, we will verify that under the basic assumption (2.2) and $0<\rho \leq \rho_{0} \leq 3, D_{\rho}$ is convex and $B_{\rho / 2} \subset D_{\rho} \subset B_{2 \rho}$. In Section 6, we will also express the graph $\psi_{z}\left(M_{\rho_{0}}\right)$ for $z \in M_{\rho_{0}}$ as

$$
\eta_{*}^{n}=\sum_{|I|=2} h_{I}\left(\xi_{*}, x\right) \xi_{*}^{I}, \quad \xi_{*}=\left(\operatorname{Re} \zeta_{*}, \operatorname{Im} \zeta_{*}^{\prime}\right) .
$$


The latter will be used to show $\left|r_{z} \cdot(\zeta-z)\right| \geq C^{-1}(\rho \sigma)^{2}$ for $z \in M_{(1-\sigma) \rho}$ and $\zeta \in \partial M_{\rho}$. In Sections 9 and 10, we derive the $\mathcal{C}^{a}$-estimates. The $\frac{1}{2}$-estimate is in Section 12, following a reduction for $\mathcal{C}^{k+\frac{1}{2}}$-estimates in Section 11.

We conclude this section with a lower bound for $\left|\zeta^{n}-z^{n}\right|$. By the basic assumption (2.2), $D_{\rho_{0}}$ is relatively compact in $D$ and hence $\left(x^{n}\right)^{2}+y^{n}=\rho^{2}$ on $\partial M_{\rho}$ for $0<\rho \leq \rho_{0}$. Then the image of the projection of $\partial M_{\rho}$ in the $z^{n}$-plane is contained in the parabola $\left(x^{n}\right)^{2}+y^{n}=\rho^{2}$. Therefore, for $z \in M_{(1-\sigma) \rho}$ and $\zeta \in \partial M_{\rho}$ with $\rho \leq \rho_{0}(\leq 3)$, we obtain

$$
\left|\zeta^{n}-z^{n}\right| \geq C^{-1} \rho^{2} \sigma
$$

\section{Domains and images under the transformation}

Recall that our real hypersurface $M$ is given by (2.1)-(2.2), and

$$
M_{\rho}=M \cap\left\{\left(x^{n}\right)^{2}+y^{n}<\rho^{2}\right\}, \quad D_{\rho}=\pi\left(M_{\rho}\right)=\left\{x \in D:|x|^{2}+\hat{r}(x)<\rho^{2}\right\} .
$$

Lemma 5.1. Let $M$ satisfy (2.2). Suppose that $0<\rho \leq \rho_{0}$. Then $\overline{D_{\rho}}$ is strictly convex with $\mathcal{C}^{2}$ boundary. Also,

$$
B_{(1-c \epsilon) \rho} \subset D_{\rho} \subset B_{(1+c \epsilon) \rho}, \quad C^{-1} \rho \sigma \leq \operatorname{dist}\left(\partial D_{(1-\sigma) \rho}, \partial D_{\rho}\right) \leq C \rho \sigma .
$$

Moreover, constants $C_{0}, c, C$ are independent of $\rho$ and $\hat{r}$.

Proof. Let $\epsilon=\|\hat{r}\|_{\rho_{0}, 2}<C_{0}^{-1}$. The strict convexity follows from the positivity of the Hessian of $\phi(x)=\left(x^{n}\right)^{2}+y^{n}=|x|^{2}+\hat{r}(x)$ on $\overline{D_{\rho}}$. Since $0 \in D_{\rho}$, then $|\hat{r}(x)| \leq C \epsilon|x|^{2}$ on $D_{\rho}$. Now,

$$
(1-C \epsilon)|x|^{2} \leq \phi(x) \leq(1+C \epsilon)|x|^{2} .
$$

In particular, for a possibly larger $C$, we have $B_{(1-C \epsilon)^{1 / 2} \rho} \subset D_{\rho} \subset B_{(1+C \epsilon)^{1 / 2} \rho}$, since $\overline{D_{\rho_{0}}} \subset D$ implies that $\phi=\rho^{2}$ on $\partial D_{\rho}$ for all $0<\rho \leq \rho_{0}$.

Let $x \in \partial D_{(1-\sigma) \rho}$. Then $\phi(x)=((1-\sigma) \rho)^{2}$ and $\frac{1}{2}(1-\sigma) \rho<|x|<$ $2(1-\sigma) \rho$. For $y \in D_{\rho}$, we have $|\phi(y)-\phi(x)| \leq C\left\|\partial^{1} \phi\right\|_{\rho, 0}|y-x|$. We get

$$
|\phi(y)| \leq(1-\sigma)^{2} \rho^{2}+C\left\|\partial^{1} \phi\right\|_{\rho, 0}|y-x| \leq(1-\sigma)^{2} \rho^{2}+C \rho|y-x| .
$$

Hence, $\phi(y)<\rho$ for $|y-x| \leq C^{-1} \rho \sigma$. This shows that $\operatorname{dist}\left(\partial D_{(1-\sigma) \rho}, \partial D_{\rho}\right) \geq$ $C^{-1} \rho \sigma$. On the other hand, if $y=(1+t) x \in D_{\rho}$ and $t>0$, applying the meanvalue-theorem to $\phi((1+s) x)$ for $0 \leq s \leq t$ yields

$$
\begin{aligned}
\phi(y) & \geq((1-\sigma) \rho)^{2}+x \cdot(y-x)-C \epsilon|y||y-x| \\
& \geq \rho^{2}(1-\sigma)^{2}+t|x|^{2}-C^{\prime} \epsilon \rho t|x| \\
& \geq \rho^{2}\left\{(1-\sigma)^{2}+\frac{1}{4} t(1-\sigma)^{2}-2 C^{\prime \prime} \epsilon t\right\} .
\end{aligned}
$$


This show that $\phi((1+t) x)>\rho^{2}$ if $(1+t) x \in D_{\rho}$ and $t>C \sigma$, a contradiction. Therefore, $\operatorname{dist}\left(\partial D_{(1-\sigma) \rho}, \partial D_{\rho}\right) \leq C \rho \sigma$.

Recall the approximate Heisenberg transformation

$$
\psi_{z}: \zeta_{*}^{\prime}=\zeta^{\prime}-z^{\prime}, \quad \zeta_{*}^{n}=-2 i r_{z} \cdot(\zeta-z)
$$

Before applying $\psi_{z}$ to kernels $k_{a b}^{j}(\zeta, z)$, we need to know how it transforms $M_{\rho}$. Recall our notation $x \in \mathbb{R}^{2 n-1}$ and $\xi \in \mathbb{R}^{2 n-1}$. Define a map $\tilde{\psi}_{x}$ by relations

$$
\xi_{*}=\tilde{\psi}_{x}(\xi)=\pi \psi_{z}(\zeta), \quad \zeta, z \in M_{\rho_{0}} .
$$

Set $\Psi(\xi, x)=\left(\tilde{\psi}_{x}(\xi), x\right)$. We have the following.

Lemma 5.2. Let $M: y^{n}=\left|z^{\prime}\right|^{2}+\hat{r}\left(z^{\prime}, x^{n}\right)$ satisfy (2.2) with $0<\rho_{0} \leq 3$. There exist constants $C, C_{m}$, independent of $\hat{r}, \rho$ and $\rho_{0}$, such that the following hold.

(i) If $x \in D_{\rho}$ and $0<\rho \leq \rho_{0}$ then

$$
\tilde{\psi}_{x}\left(D_{\rho}\right) \subset B_{9 \rho}, \quad \Psi\left(D_{\rho} \times D_{\rho}\right) \subset B_{9 \rho} \times D_{\rho} .
$$

(ii) For $u, v \in D_{\rho_{0}} \times D_{\rho_{0}}$,

$$
C^{-1}|v-u| \leq|\Psi(v)-\Psi(u)| \leq C|v-u| .
$$

In particular, if $z \in M_{\rho_{0}}$ then $\psi_{z}\left(M_{\rho_{0}}\right)$ is a graph over $\pi \psi_{z}\left(M_{\rho_{0}}\right)$.

Proof. Let $R(x)=\left|z^{\prime}\right|^{2}+\hat{r}(x)$. By (2.2), $\overline{D_{\rho_{0}}} \subset D$ and $\epsilon=\|\hat{r}\|_{\rho_{0}, 2}<C_{0}^{-1}$. For brevity, set $M=M_{\rho_{0}}$.

(i). Assume that $0<\rho \leq \rho_{0}$. Since $D_{\rho_{0}}$ is convex, we have $|\hat{r}(x)| \leq C \epsilon|x|^{2}$ on $D_{\rho_{0}}$. The map $\tilde{\psi}_{x}$ is defined by $\zeta_{*}^{\prime}=\zeta^{\prime}-z^{\prime}$ and

$$
\begin{aligned}
\xi_{*}^{n} & =\xi^{n}-x^{n}+\hat{r}_{x^{n}}(R(\xi)-R(x))+2 \operatorname{Im}\left[R_{z^{\prime}} \cdot\left(\zeta^{\prime}-z^{\prime}\right)\right] \\
& =\xi^{n}-x^{n}+2 \operatorname{Im}\left(\overline{z^{\prime}} \cdot \zeta^{\prime}\right)+\hat{r}_{x^{n}}(R(\xi)-R(x))+2 \operatorname{Im}\left[\hat{r}_{z^{\prime}} \cdot\left(\zeta^{\prime}-z^{\prime}\right)\right] .
\end{aligned}
$$

Let $\xi, x$ be in $D_{\rho}$. Recall that $D_{\rho} \subset B_{(1+C \epsilon) \rho}$. We have

$$
\begin{gathered}
|x|<(1+C \epsilon) \rho, \quad|\xi|<(1+C \epsilon) \rho, \quad\left|\hat{r}_{x^{n}}\right| \leq \epsilon, \quad\left|\hat{r}_{z^{\prime}}\right| \leq \epsilon, \\
|R(\xi)| \leq\left|\zeta^{\prime}\right|^{2}+C \epsilon|\xi|^{2}<\left(1+C^{\prime} \epsilon\right) \rho^{2} .
\end{gathered}
$$

Thus, $\left|\zeta_{*}^{\prime}\right|^{2}=\left|\zeta^{\prime}-z^{\prime}\right|^{2} \leq 2\left|\zeta^{\prime}\right|^{2}+2\left|z^{\prime}\right|^{2}<4(1+C \epsilon) \rho^{2}$; by (5.1), $\left|\xi_{*}^{n}\right|^{2} \leq$ $\left(2 \rho+2 \rho^{2}+C \epsilon \rho\right)^{2}$. Since $2 \rho^{2} \leq 6 \rho$ then

$$
\left|\zeta_{*}^{\prime}\right|^{2}+\left|\xi_{*}^{n}\right|^{2} \leq 4(1+C \epsilon) \rho^{2}+\left(2 \rho+2 \rho^{2}+C \epsilon \rho\right)^{2}<(9 \rho)^{2},
$$

if $\epsilon$ is sufficiently small. We get $\tilde{\psi}_{x}\left(D_{\rho}\right) \subset B_{9 \rho}$. 
(ii). It is obvious that the Lipschitz constant of $\Psi$ on the convex domain $D_{\rho_{0}} \times D_{\rho_{0}}$ is bounded by some $C$. Let $\xi, x, \tilde{\xi}, \tilde{x}$ be in $D_{\rho_{0}}$. We need to show that $\mid \Psi(\tilde{\xi}, \tilde{x})-$ $\Psi(\xi, x)\left|\geq C^{-1}\right|(\tilde{\xi}, \tilde{x})-(\xi, x) \mid$. Write $\xi=\left(\zeta^{\prime}, \xi^{n}\right), x=\left(z^{\prime}, x^{n}\right)$. Then

$$
|\Psi(\tilde{\xi}, \tilde{x})-\Psi(\xi, x)| \geq\left|\left(\tilde{\zeta}^{\prime}-\tilde{z}^{\prime}-\zeta^{\prime}+z^{\prime}, \tilde{x}-x\right)\right| \geq\left|\left(\tilde{\zeta}^{\prime}-\zeta^{\prime}, \tilde{x}-x\right)\right| / C .
$$

Set $\tilde{\xi}_{*}=\tilde{\psi}_{\tilde{x}}(\tilde{\xi})$ and $\xi_{*}=\tilde{\psi}_{x}(\xi)$. It suffices to show that

$$
\left|\tilde{\xi}_{*}^{n}-\xi_{*}^{n}\right| \geq\left|\tilde{\xi}^{n}-\xi^{n}\right| / C
$$

if $\left|\left(\tilde{\zeta}^{\prime}-\zeta^{\prime}, \tilde{x}-x\right)\right|<\frac{1}{48}\left|\tilde{\xi}^{n}-\xi^{n}\right|$. Assume that the latter holds. Recall that $0<\rho_{0} \leq 3$ and $D_{\rho_{0}} \subset B_{2 \rho_{0}}$. We have $\max \left\{\left|z^{\prime}\right|,\left|\tilde{\zeta}^{\prime}\right|\right\}<2 \rho_{0}$. Now the second identity in (5.1) implies that

$$
\begin{aligned}
\left|\tilde{\xi}_{*}^{n}-\xi_{*}^{n}\right| & \geq\left|\tilde{\xi}^{n}-\xi^{n}\right|-\left|\tilde{x}^{n}-x^{n}\right|-2\left|\tilde{\zeta}^{\prime}\right|\left|\tilde{z}^{\prime}-z^{\prime}\right|-2\left|z^{\prime}\right|\left|\tilde{\zeta}^{\prime}-\zeta^{\prime}\right|-C \epsilon|(\tilde{\xi}-\xi, \tilde{x}-x)| \\
& \geq\left(1-\frac{1}{48}\right)\left|\tilde{\xi}^{n}-\xi^{n}\right|-8 \rho_{0}\left(\left|\tilde{\zeta}^{\prime}-\zeta^{\prime}\right|+\left|\tilde{z}^{\prime}-z^{\prime}\right|\right)-C^{\prime} \epsilon\left|\tilde{\xi}^{n}-\xi^{n}\right| .
\end{aligned}
$$

Thus, $\left|\tilde{\xi}_{*}^{n}-\xi_{*}^{n}\right| \geq\left(1-\frac{1}{48}-\frac{8 \cdot 3 \cdot \sqrt{2}}{48}-C^{\prime} \epsilon\right)\left|\tilde{\xi}^{n}-\xi^{n}\right| \geq\left|\tilde{\xi}^{n}-\xi^{n}\right| / 4$

That $\psi_{z}\left(M_{\rho_{0}}\right)$ is a graph follows from the injectivity of $\Psi$.

\section{Estimates on $r_{z} \cdot(\zeta-z), r_{\zeta}-r_{z}$ and $\psi_{z}(M)$ via Taylor's theorem}

To transform $k(\zeta, z)$ via $\psi_{z}$, we need expansions of $r_{z} \cdot(\zeta-z), r_{\zeta} \cdot(\zeta-z)$ and $r_{\zeta^{j}}-r_{z^{j}}$ in new variables $\xi_{*}$. We will find these expansions via Taylor's theorem.

Let us recall Taylor's theorem. Assume that $0<\rho \leq \rho_{0} \leq 3$ and $\hat{r}$ satisfies (2.2). So $D_{\rho}$ is strictly convex. If $f$ is a complex-valued function on the convex set $D_{\rho}$, we define $\mathcal{R}_{k} f$ and $\mathcal{R}_{I} f$ on $D_{\rho} \times D_{\rho}$ by

$$
\begin{aligned}
\mathcal{R}_{k} f(y, x) & \equiv f(y)-\left.\sum_{0 \leq j \leq k-1} \frac{1}{j !} \partial_{t}^{j}\right|_{t=0} f(x+t(y-x)) \\
& =\frac{1}{(k-1) !} \int_{0}^{1}(1-t)^{k-1} \partial_{t}^{k} f(x+t(y-x)) d t \\
& =\sum_{|I|=k} \mathcal{R}_{I} f(y, x)(y-x)^{I} .
\end{aligned}
$$

Denote by $\mathcal{R}^{k} f$ the set of coefficients $\mathcal{R}_{I} f$ with $|I|=k$. For any real number $a \geq 0$,

$$
\left\|\mathcal{R}_{I} f\right\|_{D_{\rho} \times D_{\rho}, a} \leq C_{a+|I|}\|f\|_{\rho, a+|I|} .
$$

We will also need to express the remainders in $\xi_{*}=\left(\operatorname{Re} \zeta_{*}, \operatorname{Im} \zeta_{*}^{\prime}\right)$. Let $z \in M_{\rho_{0}}$ and $\pi(z)=x \in D_{\rho_{0}}$. By Lemma 5.2, $\psi_{z}\left(M_{\rho_{0}}\right)$ is a graph

$$
\eta_{*}^{n}=h\left(\xi_{*}, x\right), \quad \xi_{*} \in \tilde{\psi}_{x}\left(D_{\rho_{0}}\right) .
$$


By $\zeta^{\prime}-z^{\prime}=\zeta_{*}^{\prime}$ and $\zeta_{*}^{n}=-2 i r_{z} \cdot(\zeta-z)$, we have

$$
\zeta_{*}^{n}=\zeta^{n}-z^{n}-i \hat{r}_{x^{n}}\left(\zeta^{n}-z^{n}\right)-2 i R_{z^{\prime}} \cdot \zeta_{*}^{\prime}
$$

Here $R(x)=\left|z^{\prime}\right|^{2}+\hat{r}(x)$. Computing the real and imaginary parts, we get

$$
\begin{aligned}
& \xi_{*}^{n}=\xi^{n}-x^{n}+\hat{r}_{x^{n}}(R(\xi)-R(x))+2 \operatorname{Im}\left(R_{z^{\prime}} \cdot \zeta_{*}^{\prime}\right), \\
& \eta_{*}^{n}=\left|\zeta_{*}^{\prime}\right|^{2}+\hat{r}(\xi)-\hat{r}(x)-\hat{r}_{x^{n}}\left(\xi^{n}-x^{n}\right)-2 \operatorname{Re}\left(\hat{r}_{z^{\prime}} \cdot \zeta_{*}^{\prime}\right) .
\end{aligned}
$$

In (6.2), replace $R(\xi)-R(x)$ by

$$
\sum_{|I|=1} \mathcal{R}_{I} R(\xi, x)(\xi-x)^{I}=\sum_{|I|=1} \mathcal{R}_{I} R(\xi, x)\left(\xi_{*}^{\prime}, \xi^{n}-x^{n}\right)^{I}
$$

and then solve for $\xi^{n}-x^{n}$. We get

$$
\xi^{n}-x^{n}=\sum_{|I|=1} p_{I}(\xi, x) \xi_{*}^{I}
$$

where $p_{I}(\xi, x)$ are of the form

$$
p\left(\xi, x, \frac{1}{1+\hat{r}_{x^{n}} \mathcal{R}_{\left(0^{\prime}, 1\right)} \hat{r}(\xi, x)}, \partial \hat{r}(x), \mathcal{R}^{1} \hat{r}(\xi, x)\right)
$$

for some polynomials $p$. With these polynomials $p$, introduce notation

$$
\mathcal{R}_{*}^{i} g=\sum_{|I|=i} p\left(\xi, x, \frac{1}{1+\hat{r}_{x^{n}} \mathcal{R}_{\left(0^{\prime}, 1\right)} \hat{r}(\xi, x)}, \partial \hat{r}(x), \mathcal{R}^{1} \hat{r}(\xi, x)\right) \mathcal{R}_{I} g(\xi, x) .
$$

Note that reappearing $p, \mathcal{R}_{*}^{i} g$ may be different. We now express Taylor remainders in variables $\zeta_{*}^{\prime}, \xi_{*}^{n}$ as follows

$$
\begin{gathered}
\mathcal{R}_{k} f(\xi, x)=\sum_{|L|=k} \mathcal{R}_{L} f(\xi, x)(\xi-x)^{L}=\sum_{|L|=k} \mathcal{R}_{*}^{k} f(\xi, x) \xi_{*}^{L}, \\
\left|\mathcal{R}_{*}^{l} \hat{r}(\xi, x)\right| \leq C\|\hat{r}\|_{\rho_{0}, 2}, \quad l \leq 2, \quad \xi, x \in D_{\rho_{0}} .
\end{gathered}
$$

We now apply notation (6.5). Using (6.4) in (6.3) we get

$$
\begin{gathered}
\eta_{*}^{n}=\left|\zeta_{*}^{\prime}\right|^{2}+\sum_{|I|=1} \mathcal{R}_{*}^{1} \hat{r}(\xi, x) \xi_{*}^{I} \\
\eta_{*}^{n}=\left|\zeta_{*}^{\prime}\right|^{2}+\mathcal{R}_{2} \hat{r}(\xi, x)=\left|\zeta_{*}^{\prime}\right|^{2}+\sum_{|I|=2} \mathcal{R}_{*}^{2} \hat{r}(\xi, x) \xi_{*}^{I}
\end{gathered}
$$

Set $r(z)=-y^{n}+\left|z^{\prime}\right|^{2}+\hat{r}\left(z^{\prime}, x^{n}\right)$. We have defined

$$
N(\zeta, z)=\left|\zeta^{\prime}-z^{\prime}\right|^{2}+2 i \operatorname{Im}\left(r_{z} \cdot(\zeta-z)\right), \quad N_{*}\left(\xi_{*}\right)=\left|\zeta_{*}^{\prime}\right|^{2}+i \xi_{*}^{n} .
$$


Recall that for $(\zeta, z) \in M_{\rho_{0}} \times M_{\rho_{0}}$ we have

$$
\begin{gathered}
-2 r_{z} \cdot(\zeta-z)=\overline{N(\zeta, z)}+\mathcal{R}_{2} \hat{r}(\xi, x), \quad 2 r_{\zeta} \cdot(\zeta-z)=N(\zeta, z)+B(\zeta, z), \\
B(\zeta, z)=2\left(\hat{r}_{\zeta}-\hat{r}_{z}\right) \cdot(\zeta-z)-\mathcal{R}_{2} \hat{r}(\xi, x) .
\end{gathered}
$$

Define

$$
\mathcal{R}_{*}^{i} \partial \hat{r}=\sum_{1 \leq j \leq n} \mathcal{R}_{*}^{i} \hat{r}_{x_{j}}+\sum_{1 \leq j<n} \mathcal{R}_{*}^{i} \hat{r}_{y_{j}}
$$

We have

$$
\begin{gathered}
\left(\hat{r}_{\zeta}-\hat{r}_{z}\right) \cdot(\zeta-z)=\sum_{|K|=2} \mathcal{R}_{*}^{1} \partial \hat{r}(\xi, x) \xi_{*}^{K}, \\
B(\zeta, z)=\sum_{|L|=2}\left(\mathcal{R}_{*}^{2} \hat{r}(\xi, x)+\mathcal{R}_{*}^{1} \partial \hat{r}(\xi, x)\right) \xi_{*}^{L}
\end{gathered}
$$

For the numerator of the kernel, we have

$$
r_{\zeta}-r_{z}=\left(\zeta_{*}^{\prime}, 0\right)+\sum_{|I|=1}\left(\mathcal{R}_{*}^{1} \partial \hat{r}, \ldots, \mathcal{R}_{*}^{1} \partial \hat{r}\right)(\xi, x) \xi_{*}^{I} .
$$

In summary, we have proved the following expansions.

Lemma 6.1. Let $M: y^{n}=\left|z^{\prime}\right|^{2}+\hat{r}\left(z^{\prime}, x^{n}\right)$ satisfy (2.2) with $0<\rho_{0} \leq 3$. Suppose that $\zeta, z \in M_{\rho_{0}}$ and $\zeta_{*}=\psi_{z}(\zeta)$. Then $\psi_{z}\left(M_{\rho_{0}}\right)$ is given by $\eta_{*}^{n}=\left|\zeta_{*}^{\prime}\right|^{2}+h\left(\xi_{*}, x\right)$. Moreover,

$$
\begin{aligned}
h\left(\xi_{*}, x\right) & =\sum_{|I|=1} \mathcal{R}_{*}^{1} \hat{r}(\xi, x) \xi_{*}^{I}=\sum_{|I|=2} \mathcal{R}_{*}^{2} \hat{r}(\xi, x) \xi_{*}^{I}, \\
-2 r_{z} \cdot(\zeta-z) & =\left|\zeta_{*}^{\prime}\right|^{2}-i \xi_{*}^{n}+\sum_{|I|=2} \mathcal{R}_{*}^{2} \hat{r}(\xi, x) \xi_{*}^{I}, \\
2 r_{\zeta} \cdot(\zeta-z) & =\left|\zeta_{*}^{\prime}\right|^{2}+i \xi_{*}^{n}+\sum_{|I|=2}\left(\mathcal{R}_{*}^{2} \hat{r}(\xi, x)+\mathcal{R}_{*}^{1} \partial \hat{r}(\xi, x)\right) \xi_{*}^{I}, \\
r_{\zeta}-r_{z} & =\left(\zeta_{*}^{\prime}, 0\right)+\sum_{|I|=1}\left(\mathcal{R}_{*}^{1} \hat{r}_{z^{1}}, \ldots, \mathcal{R}_{*}^{1} \hat{r}_{z^{n}}\right)(\xi, x) \xi_{*}^{I} .
\end{aligned}
$$

We emphasize that $\mathcal{R}_{*}^{i} g, \mathcal{R}_{*}^{i} \partial \hat{r}$, defined by (6.5) and (6.11), might be different when they reoccur.

Remark 6.2. Notice that (6.2)-(6.8) are valid if we fix $\zeta \in M_{\rho_{0}}$ and vary $z \in M_{\rho_{0}}$. Therefore, the image of $M_{\rho_{0}}$ under the map $z \rightarrow \psi_{z}(\zeta)$ is still given by (6.7) and (6.8), where $z$ varies in $M_{\rho_{0}}$.

Here are immediate consequences of the above expansions:

$$
\left|\frac{2 r_{\zeta} \cdot(\zeta-z)}{N(\zeta, z)}-1\right|<\frac{1}{2}, \quad\left|\frac{2 r_{z} \cdot(\zeta-z)}{\overline{N(\zeta, z)}}+1\right|<\frac{1}{2}, \quad \frac{|N(z, \zeta)|}{|N(\zeta, z)|}<4
$$

for $\zeta, z \in M_{\rho_{0}}$ with $\zeta \neq z$ and $\rho_{0} \leq 3$. 
Lemma 6.3. Let $M$ satisfy (2.2) and let $0<\rho \leq \rho_{0} \leq 3$. Set $d(\zeta, z)=\left|r_{z} \cdot(\zeta-z)\right|$. Then

$$
\begin{gathered}
C^{-1}|\zeta-z|^{2} \leq d(\zeta, z) \leq C(d(\zeta, v)+d(v, z)), \quad \zeta, z, v \in M_{\rho_{0}} \\
d(\zeta, z) \geq C^{-1} \rho^{2} \sigma^{2}, \quad z \in M_{(1-\sigma) \rho}, \zeta \in \partial M_{\rho}, \quad 0<\sigma<1 .
\end{gathered}
$$

Proof. $M$ is defined by $r=-y^{n}+\left|z^{\prime}\right|^{2}+\hat{r}\left(z^{\prime}, x^{n}\right)=0$ and $\epsilon=\|\hat{r}\|_{\rho_{0}, 2}<C_{0}^{-1}$. Let $\zeta, z, v$ be in $M_{\rho_{0}}$. Set $\zeta_{*}^{\prime}=\zeta^{\prime}-z^{\prime}, \zeta_{*}^{n}=-2 i r_{z} \cdot(\zeta-z)$. Recall that $D_{\rho_{0}}$ is convex.

(i). By definition,

$$
N(\zeta, z)=\left|\zeta^{\prime}-z^{\prime}\right|^{2}+2 i \operatorname{Im}\left(r_{z} \cdot(\zeta-z)\right)=\left|\zeta_{*}^{\prime}\right|^{2}+i \xi_{*}^{n}
$$

By Lemma 6.1, we have

$$
\psi_{z} M_{\rho_{0}}: \eta_{*}^{n}=\left|\zeta_{*}^{\prime}\right|^{2}+\sum_{|I|=2} \mathcal{R}_{*}^{2} \hat{r}(\xi, x) \xi_{*}^{I}
$$

On $D_{\rho_{0}} \times D_{\rho_{0}},\left|\mathcal{R}_{*}^{2} \hat{r}\right| \leq C \epsilon$, and $\left|\xi_{*}\right|<C$. Then $\left|\zeta_{*}^{n}\right|^{2}=\left|\xi_{*}^{n}\right|^{2}+\left|\eta_{*}^{n}\right|^{2} \leq C_{0}^{\prime}\left|\xi_{*}\right|^{2}$ Therefore, $\left|\zeta^{n}-z^{n}\right|^{2}=\left|\frac{i}{2 r_{z} n} \zeta_{*}^{n}-\frac{r_{z}^{\prime}}{r_{z} n} \cdot \zeta_{*}^{\prime}\right|^{2} \leq C|N(\zeta, z)|$. Also, $\left|\zeta^{\prime}-z^{\prime}\right|^{2} \leq$ $|N(\zeta, z)|$. We conclude that $|N(\zeta, z)| \geq C^{-1}|\zeta-z|^{2}$ for $\zeta, z \in M_{\rho_{0}}$. By (6.14) we have $d(\zeta, z) \geq|\zeta-z|^{2} / C$. Now

$$
\begin{aligned}
\left|r_{\zeta} \cdot(\zeta-z)\right| & \leq\left|\left(r_{\zeta}-r_{v}\right) \cdot(z-v)\right|+\left|r_{v} \cdot(z-v)\right|+\left|r_{\zeta} \cdot(v-\zeta)\right| \\
& \leq\left|r_{\zeta}-r_{v}\right|^{2}+|z-v|^{2}+d(z, v)+d(v, \zeta) \leq C(d(\zeta, v)+d(v, z)) .
\end{aligned}
$$

Thus $d(\zeta, z) \leq C^{\prime}(d(\zeta, v)+d(z, v))$.

(ii). By Lemma 5.1, $\operatorname{dist}\left(\partial D_{(1-\sigma) \rho}, \partial D_{\rho}\right) \geq C^{-1} \rho \sigma$. Then $d(\zeta, z) \geq C^{-1}(\rho \sigma)^{2}$ follows from $d(\zeta, z) \geq C^{-1}|\zeta-z|^{2}$.

\section{Outline of $\mathcal{C}^{a}$ estimates}

Let $M$ satisfy (2.1)-(2.2) and let $0<\rho \leq \rho_{0} \leq 3$. Recall from (3.10)-(3.11) that

$$
\begin{gathered}
P_{0}^{\prime} \varphi(x)=\sum_{|I|=q-1} \sum_{|J|=q} \sum_{1 \leq j \leq n} d{\overline{z^{\prime}}}^{I} \int_{D_{\rho}} A_{\bar{I}}^{j \bar{J}}(\xi, x) \frac{\varphi_{\bar{J}}(\xi)\left(r_{\zeta^{j}}-r_{z^{j}}\right)}{\left(N_{0}^{n-q} S_{0}^{q}\right)(\zeta, z)} d V(\xi), \\
P_{1}^{\prime} \varphi(x)=\sum_{|I|=q-1} \sum_{|J|=q} \sum_{\alpha, \beta=1}^{n-1} \sum_{s=1}^{2 n-1} d{\overline{z^{\prime}}}^{I} \int_{\partial D_{\rho}} \frac{B_{\bar{I} s}^{\alpha \beta \bar{J}}(\xi, x) \varphi \bar{J}(\xi)\left(r_{\zeta^{\alpha}}-r_{z^{\alpha}}\right) r_{\zeta^{\beta}}}{\left(\zeta^{n}-z^{n}\right)\left(N_{0}^{n-q-1} S_{0}^{q}\right)(\zeta, z)} d V^{s}(\xi) .
\end{gathered}
$$

Here $\zeta, z \in M_{\rho}$, and $A_{\bar{I}}^{j \bar{J}}, B_{\bar{I} s}^{\alpha \beta \bar{J}}$ are polynomials in $\left(r_{\zeta}, r_{\bar{\zeta}}, r_{\zeta \bar{\zeta}}, r_{\overline{z^{\prime}}}, r_{z^{n}}^{-1}, r_{z \bar{z}}\right)$. 
We emphasize that norms are defined on $D_{\rho}=\pi\left(M_{\rho}\right)$ via coordinates $x$. Let us indicate how to obtain $\mathcal{C}^{a}$ estimates. We will give estimates on shrinking domains $M_{(1-\sigma) \rho}$. For $\zeta \in \partial M_{\rho}$ and $z \in M_{(1-\sigma) \rho}$, we obtain $\min \left\{\left|r_{\zeta} \cdot(\zeta-z)\right|,\left|r_{z} \cdot(\zeta-z)\right|\right\} \geq$ $C^{-1}(\rho \sigma)^{2}$ by Lemma 6.3 and $\left|\zeta^{n}-z^{n}\right| \geq C^{-1} \rho^{2} \sigma$ by (4.2). This will allow us to estimate the $\mathcal{C}^{a}$-norm of boundary integral $P_{1}^{\prime} \varphi$ by passing derivatives over the integral sign and differentiating the kernels directly.

We now deal with the interior integrals $P_{0}^{\prime} \varphi$. Using a partition of unity, we can find a smooth function $\chi=\chi_{\sigma, \rho}$, which is 1 on $D_{\rho(1-\sigma / 2)}$ and zero off $D_{\rho(1-\sigma / 4)}$, such that $\|\chi\|_{\rho, a} \leq C_{a}(\rho \sigma)^{-a}$. On $D_{\rho}$, decompose

$$
\varphi_{0}=\chi \varphi, \quad \varphi_{1}=\varphi-\varphi_{0}, \quad P_{0}^{\prime} \varphi=P_{0}^{\prime} \varphi_{0}+P_{0}^{\prime} \varphi_{1} .
$$

Now $P_{0}^{\prime} \varphi_{1}$ can be estimated on $M_{(1-\sigma) \rho}$ by differentiating the kernels directly, since $\varphi_{1}$ is supported in $\overline{M_{\rho}} \backslash M_{\left(1-\frac{1}{2} \sigma\right) \rho}$. The only non-trivial integral is $P_{0}^{\prime} \varphi_{0}$, for which $\varphi_{0}$ has compact support in $M_{\left(1-\frac{1}{4} \sigma\right) \rho}$. To estimate the latter, we will apply the transformation $\psi_{z}$ for the integral and then differentiate the new integral.

The estimate for $P_{0}^{\prime} \varphi_{0}$ is the most technical part. We deal with this estimate first in Sections 8 and 9. The estimates for boundary and cutoff terms are in Section 10.

We now conclude this section with estimates of some integrals.

Lemma 7.1. Let $n \geq 2$. Let a be a real number, and $J=\left(j_{1}, \ldots, j_{2 n-1}\right)$ be a multiindex of non-negative integers. Let $\beta=\left(j_{2 n-1}+|J|-2 a\right)+2 n-1$ and $0<\rho_{1} \leq \rho_{0}<\infty$. Then

$$
\begin{gathered}
\int_{\left|z^{\prime}\right| \leq \rho_{1},\left|x^{n}\right|<\rho_{0}} \frac{\left|\left(z^{\prime}, \overline{z^{\prime}}, x^{n}\right)^{J}\right|}{\left.|| z^{\prime}\right|^{2}+\left.i x^{n}\right|^{a}} d V \leq \begin{cases}C \rho_{1}^{1+\beta}, & -1<\beta<2 n-3, \\
\tilde{C} \rho_{1}^{2 n-2} \log \left(2+\frac{\rho_{0}}{\rho_{1}^{2}}\right), & \beta \geq 2 n-3 ;\end{cases} \\
\int_{\rho_{1} \leq\left|z^{\prime}\right| \leq \rho_{0},\left|x^{n}\right|<\rho_{0}} \frac{\left|\left(z^{\prime}, \overline{z^{\prime}}, x^{n}\right)^{J}\right|}{\left.|| z^{\prime}\right|^{2}+\left.i x^{n}\right|^{a}} d V \leq \begin{cases}C\left|\rho_{1}^{1+\beta}-\rho_{0}^{1+\beta}\right|, & \beta \neq 1, \beta<2 n-3, \\
C \log \left(\rho_{0} / \rho_{1}\right), & \beta=-1, \\
\tilde{C}\left(\rho_{0}-\rho_{1}\right), & \beta \geq 2 n-3 .\end{cases}
\end{gathered}
$$

Here $C$ depends only on $n$ and $\beta$, and $\tilde{C}$ depends on $\rho_{0}$ too.

Proof. Using $\left|x^{n}\right| \leq\left|z^{\prime}\right|^{2}+\left|x^{n}\right|$ and $\left|z_{j}\right| \leq\left(\left|z^{\prime}\right|^{2}+\left|x^{n}\right|\right)^{1 / 2}$, we may assume, without changing $\beta$, that $J=0$ and

$$
b\left(z^{\prime}, x^{n}\right)=\frac{\left|\left(z^{\prime}, \overline{z^{\prime}}, x^{n}\right)^{J}\right|}{\left.|| z^{\prime}\right|^{2}+\left.i x^{n}\right|^{a}}=\frac{1}{\left(\left|z^{\prime}\right|^{2}+\left|x^{n}\right|\right)^{a}} .
$$

Assume first that $\beta=2 n-1-2 a<2 n-3$, i.e. $a>1$. Using polar coordinates, we get

$$
\int_{\left|z^{\prime}\right| \leq \rho_{1},\left|x^{n}\right| \leq \rho_{0}} b\left(z^{\prime}, x^{n}\right) d V \leq C \int_{0}^{\rho_{1}} d r \int_{0}^{\rho_{0}} \frac{r^{2 n-3} d x^{n}}{\left(r^{2}+\left|x^{n}\right|\right)^{a}} \leq C^{\prime} \int_{0}^{\rho_{1}} r^{\beta} d r .
$$


Also, $\int_{\rho_{1} \leq\left|z^{\prime}\right| \leq \rho_{0},\left|x^{n}\right|<\rho_{0}} b\left(z^{\prime}, x^{n}\right) d V \leq C^{\prime} \int_{\rho_{1}}^{\rho_{0}} r^{\beta} d r$. The estimates in this case follow.

Assume now that $\beta \geq 2 n-3$, i.e. $a \leq 1$. Reducing it to $a=1$ via $\tilde{C}$, we get

$$
\int_{\left|z^{\prime}\right| \leq \rho_{1},\left|x^{n}\right| \leq \rho_{0}} b\left(z^{\prime}, x^{n}\right) d V \leq \tilde{C} \int_{0}^{\rho_{1}} r^{2 n-3} \log \left(1+\frac{\rho_{0}}{r^{2}}\right) d r .
$$

Also, $\int_{\rho_{1} \leq\left|z^{\prime}\right| \leq \rho_{0},\left|x^{n}\right|<\rho_{0}} b\left(z^{\prime}, x^{n}\right) d V \leq \int_{\rho_{1}}^{\rho_{0}} \tilde{C} d r$. The estimates are obtained by a simple computation.

\section{New kernels and two formulae for derivatives}

In this section we express the kernels by using Lemma 6.1 and derive two formulae for derivatives of $P_{0} \varphi$, where $\varphi$ has compact support in $D_{\rho}$. The first formula will be used for $\mathcal{C}^{a}$ estimates and the second is for $\mathcal{C}^{k+\frac{1}{2}}$ estimates.

Recall that with $\zeta, z \in M_{\rho}$ the coefficients of $P_{0}^{\prime} \varphi(x)$ are sums of

$$
\mathcal{I}(x)=\int_{D_{\rho}} \varphi_{\bar{J}}(\xi) \frac{\partial_{*}^{2} r(\xi, x)\left(r_{\zeta^{j}}-r_{z^{j}}\right)}{\left(N_{0}^{a} S_{0}^{b}\right)(\zeta, z)} d V(\xi)
$$

over $1 \leq j \leq n$ and $|J|=q$, where $a=n-q$ and $b=q$. Set $f(\xi, x)=\varphi_{\bar{J}}(\xi)$.

We have defined $\partial_{*}^{2+k} r$ in Section 2. Now, CHANGE NOTATION and let

$$
\begin{gathered}
\partial_{*}^{2} r=p\left(\xi, x,\left(1+\hat{r}_{x^{n}} \hat{r}_{\xi^{n}}\right)^{-1}, r_{z^{n}}^{-1},\left(1+\hat{r}_{x^{n}} \mathcal{R}_{\left(0^{\prime}, 1\right)} \hat{r}(\xi, x)\right)^{-1}, \partial \hat{r}(x), \partial \hat{r}(\xi), \mathcal{Q}(\xi, x)\right), \\
\mathcal{Q}(\xi, x)=\left(\partial^{2} \hat{r}(x), \partial^{2} \hat{r}(\xi), \mathcal{R}_{*}^{1} \partial \hat{r}(\xi, x), \mathcal{R}_{*}^{1} \hat{r}(\xi, x), \mathcal{R}_{*}^{2} \hat{r}(\xi, x)\right),
\end{gathered}
$$

where $p$ is a polynomial. Again, $\mathcal{R}_{*}^{1} \partial \hat{r}, \mathcal{R}_{*}^{2} \hat{r}$, defined by (6.11) and (6.5), and $p$ might be different when they reoccur; for instance, $\left(\mathcal{R}_{*}^{1} r\right)^{2}$ may be the product of two different $\mathcal{R}_{*}^{1} r$ 's. Define

$$
\begin{gathered}
\partial_{*}^{2+k} r(\xi, x)=\sum \partial_{*}^{2} r \cdot \partial^{I_{1}} \mathcal{Q}_{1}(\xi, x) \cdots \partial^{I_{j}} \mathcal{Q}_{j}(\xi, x), \quad j \geq 0, \\
\partial_{*}^{2} \hat{r}(\xi, x)=\sum \partial_{*}^{2} r \cdot \mathcal{Q}_{1}(\xi, x) \cdots \mathcal{Q}_{j}(\xi, x), \quad j \geq 1,
\end{gathered}
$$

where $\sum_{l=1}^{j}\left|I_{l}\right| \leq k, \mathcal{Q}_{l} \in \mathcal{Q}$ and both sums have finitely many terms. Hence, we have simple relations

$$
\partial_{*}^{2+k} r \partial_{*}^{2+j} r=\partial_{*}^{2+k+j} r, \quad \partial^{J} \partial_{*}^{2+k} r=\partial_{*}^{2+k+|J|} r .
$$

The chain rule takes the form

$$
\begin{aligned}
\partial_{\xi_{*}, x}^{I} \Psi^{-1} & =\partial_{*}^{2} r \circ \Psi^{-1}, \quad|I|=1, \\
\partial_{\xi_{*}, x}^{J}\left\{\left(f \partial_{*}^{2+k} r\right) \circ \Psi^{-1}\right\} & =\sum_{|L| \leq|J|}\left(\partial^{L} f \cdot \partial_{*}^{2+k+|J|-|L|} r\right) \circ \Psi^{-1} .
\end{aligned}
$$


New kernels. Set $\left(\xi_{*}, x\right)=\Psi(\xi, x)$ with $\zeta, z \in M_{\rho_{0}}$. Recall that $N_{*}\left(\xi_{*}\right)=$ $\left|\xi_{*}^{\prime}\right|^{2}+i \xi_{*}^{n}$. By Lemma 6.1,

$$
\begin{gathered}
N_{0}(\zeta, z) \equiv 2 r_{\zeta} \cdot(\zeta-z)=N_{*}\left(\xi_{*}\right) \hat{T}_{1}\left(\xi_{*}, x\right), \\
S_{0}(\zeta, z) \equiv-2 r_{z} \cdot(\zeta-z)=\overline{N_{*}\left(\xi_{*}\right)} \hat{T}_{2}\left(\xi_{*}, x\right), \\
\hat{T}_{1}\left(\xi_{*}, x\right)=T_{1} \circ \Psi^{-1}\left(\xi_{*}, x\right)=1+\sum_{|J|=2} \partial_{*}^{2} \hat{r} \circ \Psi^{-1}\left(\xi_{*}, x\right) N_{*}^{-1}\left(\xi_{*}\right) \xi_{*}^{J}, \\
\hat{T}_{2}\left(\xi_{*}, x\right)=T_{2} \circ \Psi^{-1}\left(\xi_{*}, x\right)=1+\sum_{|J|=2} \partial_{*}^{2} \hat{r} \circ \Psi^{-1}\left(\xi_{*}, x\right) \overline{N_{*}^{-1}\left(\xi_{*}\right)} \xi_{*}^{J}, \\
\partial_{*}^{2} r(\zeta, z)\left(r_{\zeta^{j}}-r_{z^{j}}\right)=\sum_{|I|=1} \partial_{*}^{2} r \circ \Psi^{-1}\left(\xi_{*}, x\right) \xi_{*}^{I} .
\end{gathered}
$$

Note that $\left|\partial_{*}^{2} \hat{r}\right| \leq C \epsilon$ and $\left|\hat{T}_{j}\left(\xi_{*}, x\right)-1\right|<1 / 2$ when $\xi_{*} \neq 0$. Thus, we obtain

$$
\begin{gathered}
\frac{\partial_{*}^{2} r(\zeta, z)\left(r_{\zeta^{j}}-r_{z^{j}}\right)}{\left(N_{0}^{a} S_{0}^{b}\right)(\zeta, z)}=\sum_{|I|=1}\left\{\partial_{*}^{2} r \circ \Psi^{-1} \cdot \hat{T}_{1}^{-a} \hat{T}_{2}^{-b}\right\}\left(\xi_{*}, x\right) \hat{k}_{a b}^{I}\left(\xi_{*}\right), \\
\hat{k}_{a b}^{I}\left(\xi_{*}\right)=\xi_{*}^{I} N_{*}^{-a}\left(\xi_{*}\right) \overline{N_{*}^{-b}\left(\xi_{*}\right)}, \quad a=n-q, b=q .
\end{gathered}
$$

First formula of derivatives of $\mathcal{I}$. Recall that $\Psi(\xi, x)=\left(\tilde{\psi}_{x}(\xi), x\right), \xi_{*}=\tilde{\psi}_{x}(\xi)$ and $\left(\tilde{\psi}_{x}^{*} d V\right)\left(\xi_{*}\right)=\left(\partial_{*}^{1} r\right) \circ \Psi^{-1}\left(\xi_{*}, x\right) d V\left(\xi_{*}\right)$. By (8.4) and $\partial_{*}^{2} r \partial_{*}^{1} r=\partial_{*}^{2} r$, we obtain

$$
\mathcal{I}(x)=\sum_{|I|=1} \int_{\tilde{\psi}_{x}\left(D_{\rho}\right)}\left\{\left(f \partial_{*}^{2} r\right) \circ \Psi^{-1} \cdot \hat{T}_{1}^{-a} \hat{T}_{2}^{-b}\right\}\left(\xi_{*}, x\right) \hat{k}_{a b}^{I}\left(\xi_{*}\right) d V\left(\xi_{*}\right),
$$

where $a=n-q, b=q$. By Lemma 7.1 with $\beta \geq 0, \hat{k}_{a b}^{I} \in L_{\text {loc }}^{1}$. For each $x \in D_{\rho}$, the integrand has compact support in $\tilde{\psi}_{x}\left(D_{\rho}\right) \subset B_{9 \rho}$. To compute $\partial^{k} \mathcal{I}(x)$, we extend the integrand of $\mathcal{I}(x)$ to be zero on $B_{9 \rho} \backslash \tilde{\psi}_{x}\left(D_{\rho}\right)$. The integral is over the fixed domain $B_{9}$. So we can interchange the integral sign with $\partial_{x}$. The derivatives of $\mathcal{I}$ have the form

$$
\begin{aligned}
& \partial^{K} \mathcal{I}(x)=\sum_{j+k^{\prime}+l+m=|K|} \sum_{|J|=j} \sum_{\left|K^{\prime}\right|=k^{\prime}} \sum_{|L|=l} \sum_{|I|=1} \\
& \int_{B_{9 \rho}}\left\{\left(\partial^{L} f \partial_{*}^{2+m} r\right) \circ \Psi^{-1} \cdot \partial_{x}^{J} \hat{T}_{1}^{-a} \cdot \partial_{x}^{K^{\prime}} \hat{T}_{2}^{-b}\right\}\left(\xi_{*}, x\right) \cdot \hat{k}_{a b}^{I}\left(\xi_{*}\right) d V\left(\xi_{*}\right) .
\end{aligned}
$$

By (8.2), the first-order $x$-derivatives of $\hat{T}_{1}^{-a}$ have the form

$$
\begin{aligned}
\partial_{x}^{I} \hat{T}_{1}^{-a}\left(\xi_{*}, x\right) & =\sum_{\left|J^{\prime}\right|=1} \sum_{\left|L^{\prime}\right|=2}\left\{\hat{T}_{1}^{-a-1} \partial_{x}^{J^{\prime}}\left(\partial_{*}^{2} r \circ \Psi^{-1}\right)\right\}\left(\xi_{*}, x\right) N_{*}^{-1}\left(\xi_{*}\right) \xi_{*}^{L^{\prime}} \\
& =\sum_{\left|L^{\prime}\right|=2}\left(\partial_{*}^{3} r \circ \Psi^{-1} \hat{T}_{1}^{-a-1}\right)\left(\xi_{*}, x\right) N_{*}^{-1}\left(\xi_{*}\right) \xi_{*}^{L^{\prime}}
\end{aligned}
$$


Take derivatives consecutively and use the product rule. We can write

$$
\partial_{x}^{J} \hat{T}_{1}^{-a}\left(\xi_{*}, x\right)=\sum_{s \leq|J|} \sum_{\left|L^{\prime}\right|=2 s}\left\{\left(\partial_{*}^{2+|J|} r\right) \circ \Psi^{-1} \cdot \hat{T}_{1}^{-a-s}\right\}\left(\xi_{*}, x\right) \frac{\xi_{*}^{L^{\prime}}}{N_{*}^{s}\left(\xi_{*}\right)} .
$$

Analogously, $\partial_{x}^{K^{\prime}} \hat{T}_{2}^{-b}\left(\left|K^{\prime}\right|=k^{\prime}\right)$ can be written as

$$
\partial_{x}^{K^{\prime}} \hat{T}_{2}^{-b}\left(\xi_{*}, x\right)=\sum_{t \leq\left|K^{\prime}\right|} \sum_{\left|L^{\prime \prime}\right|=2 t}\left\{\left(\partial_{*}^{2+\left|K^{\prime}\right|} r\right) \circ \Psi^{-1} \cdot \hat{T}_{2}^{-b-t}\right\}\left(\xi_{*}, x\right) \frac{\xi_{*}^{L^{\prime \prime}}}{\overline{N_{*}^{t}\left(\xi_{*}\right)}}
$$

Let $I^{\prime}=I+L^{\prime}+L^{\prime \prime}, a^{\prime}=a+s, b^{\prime}=b+t$. We have

$N_{*}^{-s}\left(\xi_{*}\right) \xi_{*}^{L^{\prime}} \overline{N_{*}^{-t}\left(\xi_{*}\right)} \xi_{*}^{L^{\prime \prime}} \hat{k}_{a b}^{I}=\hat{k}_{a^{\prime} b^{\prime}}^{I^{\prime}}, \quad 2 a^{\prime}+2 b^{\prime}-\left|I^{\prime}\right|=2 n-1, a^{\prime}+b^{\prime} \leq n+|K|$.

By (8.8)-(8.9), we get

$$
\begin{aligned}
\partial^{K} \mathcal{I}(x)= & \sum_{|L| \leq|K|} \sum_{a \leq a^{\prime} \leq a+|K|} \sum_{b \leq b^{\prime} \leq b+|K|} \sum_{2 a^{\prime}+2 b^{\prime}-\left|I^{\prime}\right|=2 n-1} \\
& \int_{B_{9 \rho}} \frac{\left(\partial^{L} f \partial_{*}^{2+|K|-|L|} r\right) \circ \Psi^{-1}\left(\xi_{*}, x\right)}{\left(\hat{T}_{1}^{a^{\prime}} \hat{T}_{2}^{b^{\prime}}\right)\left(\xi_{*}, x\right)} \hat{k}_{a^{\prime} b^{\prime}}^{I^{\prime}}\left(\xi_{*}\right) d V\left(\xi_{*}\right) .
\end{aligned}
$$

Recall that $f(\xi, x)=\varphi_{\bar{J}}(\xi)$ has compact support in $D_{\rho}$. Since $\left|I^{\prime}\right|=2 a^{\prime}+$ $2 b^{\prime}-2 n+1$, Lemma 7.1 with $\beta \geq 0$ implies $\hat{k}_{a^{\prime} b^{\prime}}^{I^{\prime}} \in L_{\text {loc }}^{1}$. By the dominated convergence theorem, we see that $\partial^{k} \mathcal{I}$ are continuous. Note that this also implies that if $\hat{r} \in \mathcal{C}^{k+2}\left(\bar{D}_{\rho_{0}}\right)$ and $\varphi \in C^{k}\left(\bar{D}_{\rho_{0}}\right)$, then $P^{\prime} \varphi \in \mathcal{C}^{k}\left(D_{\rho_{0}}\right)$.

Second formula of derivatives of $\mathcal{I}$. We return to the original coordinates by letting $\left(\xi_{*}, x\right)=\Psi(\xi, x)$ and $\zeta, z \in M_{\rho}$. So $d V\left(\xi_{*}\right)=\partial_{*}^{1} r d V(\xi)$. Also

$$
\begin{gathered}
\hat{T}_{1}^{-a^{\prime}} \circ \Psi(\xi, x)=N^{a^{\prime}}(\zeta, z) N_{0}^{-a^{\prime}}(\zeta, z), \quad \hat{T}_{2}^{-b^{\prime}} \circ \Psi(\xi, x)=\overline{N^{b^{\prime}}(\zeta, z)} S_{0}^{-b^{\prime}}(\zeta, z), \\
\hat{k}_{a^{\prime} b^{\prime}}^{I^{\prime}} \circ \tilde{\psi}_{x}(\xi)=\frac{\left(\operatorname{Re}\left(\zeta^{\prime}-z^{\prime}\right), \operatorname{Im}\left(\zeta^{\prime}-z^{\prime}\right), 2 \operatorname{Im}\left(r_{z} \cdot(\zeta-z)\right)\right)^{I^{\prime}}}{N(\zeta, z)^{a^{\prime}} \overline{N^{b^{\prime}}(\zeta, z)}} .
\end{gathered}
$$

Multiply the same sides of the three identities and expand the last numerator. Then $\left\{\hat{T}_{1}^{-a^{\prime}} \hat{T}_{2}^{-b^{\prime}} \hat{k}_{a^{\prime} b^{\prime}}^{I^{\prime}}\right\} \circ \Psi$ is a linear combination of

$$
k_{a^{\prime} b^{\prime}}^{I^{\prime \prime}}(\xi, x) \stackrel{\operatorname{def}}{=} \frac{\left(\zeta^{\prime}-z^{\prime}, \overline{\zeta^{\prime}-z^{\prime}}, \operatorname{Im}\left(r_{z} \cdot(\zeta-z)\right)\right)^{I^{\prime \prime}}}{N_{0}^{a^{\prime}}(\zeta, z) S_{0}^{b^{\prime}}(\zeta, z)} .
$$

Since $\left|I^{\prime \prime}\right|=\left|I^{\prime}\right|=2 a^{\prime}+2 b^{\prime}+1-2 n$, then

$$
\left|I^{\prime \prime}\right|-2 a^{\prime}-2 b^{\prime}-1+2 n=0, \quad\left|I^{\prime \prime}\right| \leq 2|K|+1, a^{\prime}+b^{\prime} \leq n+|K| .
$$


By (8.10), derivatives of $\mathcal{I}(x)$ have the form

$$
\begin{aligned}
\partial^{K} \mathcal{I}(x)= & \sum_{|L| \leq|K|} \sum_{a \leq a^{\prime} \leq a+|K|} \sum_{b \leq b^{\prime} \leq b+|K|} \sum_{1+2 a^{\prime}+2 b^{\prime}-2 n \leq\left|I^{\prime \prime}\right| \leq 2|K|+1} \\
& \int_{D_{\rho}} \partial^{L} \varphi_{\bar{J}}(\xi) \partial_{*}^{2+|K|-|L|} r(\xi, x) k_{a^{\prime} b^{\prime}}^{I^{\prime \prime}}(\xi, x) d V(\xi) .
\end{aligned}
$$

Here $\varphi_{\bar{J}}$ has compact support in $D_{\rho}$. Obviously, the $\partial^{L} \varphi_{\bar{J}}$ in (8.12) do not depend on $x$. This simple observation will be crucial for the $\frac{1}{2}$-estimate.

The reader might want to acquaint with the counting scheme in Section 2 and Hölder inequalities on domains $D_{\rho}$ in Appendix A; see Proposition A.5.

\section{9. $\mathcal{C}^{a}$-estimates, case of compact support}

In this section, we derive the $\mathcal{C}^{a}$-estimate for $P_{0} \varphi_{0}$ where

$$
\varphi_{0}=\chi \varphi, \quad\|\chi\|_{\rho, a} \leq C_{a}(\rho \sigma)^{-a}
$$

and $\chi$ is supported in $D_{\rho}$. We also derive an estimate for $P_{0} \varphi$ when $\varphi$ itself has compact support in $D_{\rho}$.

Proposition 9.1. Let $k \geq 0$ be an integer and $0 \leq \alpha<1$. Let $M: y^{n}=\left|z^{\prime}\right|^{2}+$ $\hat{r}\left(z^{\prime}, x^{n}\right)$ satisfy (2.2) and $0<\rho \leq \rho_{0} \leq 3$. Let $\varphi_{0}$ be a tangential form as in (9.1). Then

$$
\left\|P_{0}^{\prime} \varphi_{0}\right\|_{\rho, k+\alpha} \leq C_{k} \rho^{1-k-\alpha} \sigma^{-k-\alpha}\left(\|\varphi\|_{\rho, k+\alpha}+\|\varphi\|_{\rho, 0}\|\hat{r}\|_{\rho, k+2+\alpha}\right) .
$$

If $\varphi$ has compact support in $D_{\rho}$ and is tangential, then

$$
\left\|P_{0}^{\prime} \varphi\right\|_{\rho, k+\alpha} \leq C_{k} \rho^{1-k-\alpha}\left(\|\varphi\|_{\rho, k+\alpha}+\|\varphi\|_{\rho, 0}\|\hat{r}\|_{\rho, k+2+\alpha}\right) .
$$

The same estimate holds for $Q_{0}^{\prime}$.

Proof. Recall that by applying (8.10) to $f=\chi \varphi_{\bar{L}}, k$-th derivatives of a coefficient of $P_{0}^{\prime} \varphi_{0}$ are sums of finitely many

$$
\mathcal{I}_{k}(x)=\int_{B_{9 \rho} \rho} \frac{u \circ \Psi^{-1}\left(\xi_{*}, x\right)}{\hat{T}_{1}^{a^{\prime}} \hat{T}_{2}^{b^{\prime}}\left(\xi_{*}, x\right)} \hat{k}_{a^{\prime} b^{\prime}}^{I^{\prime}}\left(\xi_{*}\right) d V\left(\xi_{*}\right) .
$$

Here $a=q, b=n-q, a \leq a^{\prime} \leq a+k, b \leq b^{\prime} \leq b+k$ and

$$
\begin{gathered}
u(\xi, x) \stackrel{\text { def }}{=} \partial^{I} \chi(\xi) \partial^{J} \varphi_{\bar{L}}(\xi) \partial_{*}^{2+l} r(\xi, x), \quad|I|=i,|J|=j, \quad i+j+l=k, \\
\hat{k}_{a^{\prime} b^{\prime}}^{I^{\prime}}\left(\xi_{*}\right)=N^{-a^{\prime}}\left(\xi_{*}\right) \overline{N^{-b^{\prime}}\left(\xi_{*}\right) \xi_{*}^{I^{\prime}},}, \quad 2 a^{\prime}+2 b^{\prime}=\left|I^{\prime}\right|+2 n-1 .
\end{gathered}
$$

To obtain (9.2), we estimate the $\mathcal{C}^{\alpha}$-norm of $\mathcal{I}_{k}$. 
By the definition of $\partial_{*}^{2+l} r$, we have

$$
\left\|\partial_{*}^{2+l} r\right\|_{\rho, \alpha} \leq C \sum_{l_{1}+\cdots+l_{t} \leq l}\|r\|_{2+l_{1}+\alpha} \cdots\|r\|_{2+l_{t}} .
$$

Thus

$$
\begin{aligned}
& \left\|\partial^{i} \chi\right\|_{\rho, 0}\left\|\partial^{j} \varphi_{\bar{L}}\right\|_{\rho, 0}\left\|\partial_{*}^{2+l} r\right\|_{\rho, \alpha} \\
& \quad \leq C(\rho \sigma)^{-i} \sum_{l_{1}+\cdots+l_{t} \leq l}\left\|\varphi_{\bar{L}}\right\|_{\rho, j}\|r\|_{2+l_{1}+\alpha} \cdots\|r\|_{2+l_{t}} \\
& \quad \leq C^{\prime}(\rho \sigma)^{-i} \rho^{-l-j-\alpha}\left(\left\|\varphi_{\bar{L}}\right\|_{\rho, l+j+\alpha}+\|r\|_{2+l+j+\alpha}\left\|\varphi_{\bar{L}}\right\|_{\rho, 0}\right) .
\end{aligned}
$$

Here the last inequality is obtained by Proposition A.5. Also

$$
\begin{aligned}
& \left\|\partial^{i} \chi\right\|_{\rho, \alpha}\left\|\partial^{j} \varphi_{\bar{L}}\right\|_{\rho, 0}\left\|\partial_{*}^{2+l} r\right\|_{\rho, 0} \\
& \quad \leq C(\rho \sigma)^{-i-\alpha} \rho^{-l-j}\left(\left\|\varphi_{\bar{L}}\right\|_{\rho, l+j}+\|r\|_{2+l+j}\left\|\varphi_{\bar{L}}\right\|_{\rho, 0}\right), \\
& \left\|\partial^{i} \chi\right\|_{\rho, 0}\left\|\partial^{j} \varphi_{\bar{L}}\right\|_{\rho, \alpha}\left\|\partial_{*}^{2+l} r\right\|_{\rho, 0} \\
& \quad \leq \frac{C}{(\rho \sigma)^{i} \rho^{l+j+\alpha}}\left(\left\|\varphi_{\bar{L}}\right\|_{\rho, l+j+\alpha}+\|r\|_{2+l+j+\alpha}\left\|\varphi_{\bar{L}}\right\|_{\rho, 0}\right) .
\end{aligned}
$$

Therefore,

$$
\begin{array}{r}
\|u\|_{D_{\rho}^{2}, \alpha} \leq C_{k} \rho^{-k-\alpha} \sigma^{-k-\alpha}\left(\|\varphi\|_{\rho, k+\alpha}+\|r\|_{\rho, k+2+\alpha}\|\varphi\|_{\rho, 0}\right), \\
\|u\|_{D_{\rho}^{2}, 0}\|r\|_{\rho, 2+\alpha} \leq C_{k} \rho^{-k-\alpha} \sigma^{-k}\left(\|\varphi\|_{\rho, k+\alpha}+\|r\|_{\rho, k+2+\alpha}\|\varphi\|_{\rho, 0}\right) .
\end{array}
$$

By Lemma 5.2, we know that $W_{\rho}=\Psi\left(D_{\rho} \times D_{\rho}\right) \subset B_{9 \rho} \times D_{\rho}$ and

$$
\left|\Psi^{-1}(v)-\Psi^{-1}(u)\right| \leq C|v-u|, \quad u, v \in W_{\rho} .
$$

Assume that $0 \leq \alpha<1$. Fix $\xi_{*} \in B_{9 \rho} \backslash\{0\}$ and $x_{1}, x_{2} \in D_{\rho}$. Assume first that $\xi_{j}=\tilde{\psi}_{x_{j}}^{-1}\left(\xi_{*}\right)$ are in $D_{\rho}$ for $j=1,2$. First, by (9.6)

$$
\left|\xi_{2}-\xi_{1}\right| \leq C\left|x_{2}-x_{1}\right|
$$

Now by (8.2)-(8.3), we obtain $\left|\hat{T}_{j}\left(\xi_{*}, x\right)\right| \geq 1 / 4$ and

$$
\left|\hat{T}_{j}\left(\xi_{*}, x_{2}\right)-\hat{T}_{j}\left(\xi_{*}, x_{1}\right)\right| \leq C\left|\partial_{*}^{2} r\left(\xi_{2}, x_{2}\right)-\partial_{*}^{2} r\left(\xi_{1}, x_{1}\right)\right| \leq\|r\|_{\rho, 2+\alpha}\left|x_{2}-x_{1}\right|^{\alpha} .
$$

Thus

$$
\begin{aligned}
\Delta & =\left|\Delta\left(x_{2}\right)-\Delta\left(x_{1}\right)\right| \stackrel{\text { def }}{=}\left|\frac{u \circ \Psi^{-1}\left(\xi_{*}, x_{2}\right)}{\hat{T}_{1}^{a^{\prime}} \hat{T}_{2}^{b^{\prime}}\left(\xi_{*}, x_{2}\right)}-\frac{u \circ \Psi^{-1}\left(\xi_{*}, x_{1}\right)}{\hat{T}_{1}^{a^{\prime}} \hat{T}_{2}^{b^{\prime}}\left(\xi_{*}, x_{1}\right)}\right| \\
& \leq C\|u\|_{\rho, \alpha}\left|x_{2}-x_{1}\right|^{\alpha}+C\left|u\left(\xi_{2}, x_{2}\right)\left(\left(\partial_{*}^{2} r\right)\left(\xi_{2}, x_{2}\right)-\left(\partial_{*}^{2} r\right)\left(\xi_{1}, x_{1}\right)\right)\right| \\
& \leq C\left(\|u\|_{\rho, \alpha}+\|u\|_{\rho, 0}\|\hat{r}\|_{\rho, 2+\alpha}\right)\left|x_{2}-x_{1}\right|^{\alpha} .
\end{aligned}
$$


By (9.4)-(9.5) we get

$$
\Delta \leq C_{k} \rho^{-k-\alpha} \sigma^{-k-\alpha}\left(\|\varphi\|_{\rho, k+\alpha}+\|r\|_{\rho, k+2+\alpha}\|\varphi\|_{\rho, 0}\right)\left|x_{2}-x_{1}\right|^{\alpha} .
$$

The above holds trivially if $\xi_{1}, \xi_{2}$ are both not in $D_{\rho}$, in which case $\Delta=0$. If $\xi_{2} \in D_{\rho}$ and $\xi_{1} \notin D_{\rho}$, we replace $x_{1}$ by a point $x_{3}$ in the line segment $\left[x_{1}, x_{2}\right]$, for which $\xi_{3}=\tilde{\psi}_{x_{3}}^{-1}\left(\xi_{*}\right) \in \partial D_{\rho}$. Then $\Delta=\left|\Delta\left(x_{2}\right)\right|=\left|\Delta\left(x_{2}\right)-\Delta\left(x_{3}\right)\right|$ and (9.7) still holds. By Lemma 7.1 (with $\rho_{1}=\rho_{0}=9 \rho$ and $\beta \geq 0$ ), $\int_{B_{9 \rho}}\left|\hat{k}_{a^{\prime}, b^{\prime}}^{I^{\prime}}\right| d V \leq C \rho$. Combining the above estimates yields (9.2).

The case that $\varphi_{0}=\varphi$ is simpler, and it does not involve $\sigma$. So we can remove all powers of $\sigma$ in (9.4)-(9.5), (9.7), and (9.2). The latter becomes (9.3).

We compute the $\mathcal{C}^{\frac{1}{2}}$ norm of $\partial^{I} \chi \partial^{J} \varphi_{\bar{L}} \partial_{*}^{2+l} r$ for a later use. Here it is crucial to avoid the Hölder $\frac{1}{2}$-norm of $\partial^{J} \varphi_{\bar{L}}$.

Proposition 9.2. Let $u(\xi, x)=\partial^{I} \chi(\xi) \partial^{J} \varphi_{\bar{L}}(\xi) \partial_{*}^{2+l} r(\xi, x)$ where $\chi$ has compact support in $D_{\rho}$ and satisfies $\|\chi\|_{\rho, a} \leq C(\rho \sigma)^{-a}$. Let $|I|+|J|+l=k$. Then

$$
\|u(\xi, \cdot)\|_{\rho, \frac{1}{2}} \leq C_{k}(\rho \sigma)^{-k-\frac{1}{2}}\left(\left\|\varphi_{\bar{L}}\right\|_{\rho, k}\|r\|_{\rho, \frac{5}{2}}+\left\|\varphi_{\bar{L}}\right\|_{\rho, 0}\|\hat{r}\|_{\rho, k+\frac{5}{2}}\right) .
$$

Proof. Fix $\xi \in D_{\rho}$. The $\varphi_{\bar{L}}$ appearing in $u(\xi, x)$ depends only on $\xi$. Therefore, for $\|u(\xi, \cdot)\|_{\rho, 1 / 2}$, we only use the sup norm of $\partial^{J} \varphi_{\bar{L}}(\xi)$. Then

$$
\begin{aligned}
\|u(\xi, \cdot)\|_{\rho, 1 / 2} \leq & C_{k}\left((\rho \sigma)^{-i-\frac{1}{2}}\left\|\varphi_{\bar{L}}\right\|_{\rho, j}\|r\|_{\rho, l+2}+(\rho \sigma)^{-i}\left\|\varphi_{\bar{L}}\right\|_{\rho, j}\|r\|_{\rho, l+\frac{5}{2}}\right) \\
\leq & C_{k}^{\prime}(\rho \sigma)^{-i-\frac{1}{2}} \rho^{-j-l}\left(\left\|\varphi_{\bar{L}}\right\|_{\rho, j+l}+\left\|\varphi_{\bar{L}}\right\|_{\rho, 0}\|r\|_{\rho, 2+j+l}\right) \\
& +C_{k}^{\prime}(\rho \sigma)^{-i} \rho^{-j-l-\frac{1}{2}}\left(\left\|\varphi_{\bar{L}}\right\|_{\rho, 0}\|r\|_{\rho, j+l+\frac{5}{2}}+\left\|\varphi_{\bar{L}}\right\|_{\rho, j+l}\|r\|_{\rho, \frac{5}{2}}\right),
\end{aligned}
$$

where $|I|=i,|J|=j,|L|=l$ and the last two terms are obtained by Proposition A.5 in which we take $d_{1}=0$ and $d_{2}=\frac{5}{2}$. Simplifying yields (9.8).

\section{Boundary integrals, end of $\mathcal{C}^{a}$-estimates}

In this section we will estimate the boundary integrals $P_{1}^{\prime} \varphi$ and cutoff term $P_{0}^{\prime} \varphi_{1}$, where $\varphi_{1}$ vanishes on $D_{\left(1-\frac{1}{2} \sigma\right) \rho}$. Estimates (10.14) and (10.15) below will be used again for the $\mathcal{C}^{k+\frac{1}{2}}$ estimate.

Recall (3.10)-(3.11) that for $\zeta, z \in M_{\rho}$

$$
\begin{aligned}
& P_{0}^{\prime} \varphi(x)=\sum_{|I|=q-1} \sum_{|J|=q} \sum_{1 \leq j \leq n} d \overline{\bar{z}^{\prime}} I \int_{D_{\rho}} A_{\bar{I}}^{j \bar{J}}(\xi, x) \frac{\varphi \bar{J}(\xi)\left(r_{\zeta^{j}}-r_{z^{j}}\right)}{\left(N_{0}^{n-q} S_{0}^{q}\right)(\zeta, z)} d V(\xi), \\
& P_{1}^{\prime} \varphi(x)=\sum_{|I|=q-1} \sum_{|J|=q} \sum_{\alpha, \beta=1}^{n-1} \sum_{s=1}^{2 n-1} d \overline{z^{\prime}} I \int_{\partial D_{\rho}} \frac{B_{\bar{I} s}^{\alpha \beta \bar{J}}(\xi, x) \varphi_{\bar{J}}(\xi)\left(r_{\zeta^{\alpha}}-r_{z^{\alpha}}\right) r_{\zeta^{\beta}}}{\left(\zeta^{n}-z^{n}\right)\left(N_{0}^{n-q-1} S_{0}^{q}\right)(\zeta, z)} d V^{s}(\xi) .
\end{aligned}
$$


Here $A_{\bar{I}}^{j \bar{J}}$ and $B_{\bar{I} s}^{\alpha \beta \bar{J}}$ are polynomials in $\left(r_{\zeta}, r_{\bar{\zeta}}, r_{\zeta \bar{\zeta}}, r_{\bar{z}^{\prime}}, r_{\overline{z^{n}}}^{-1}, r_{z \bar{z}}\right)$. And $N_{0}(\zeta, z)=$ $r_{\zeta} \cdot(\zeta-z), S_{0}(\zeta, z)=r_{z} \cdot(\zeta-z)$. For $\zeta, z \in M_{\rho}$, set

$$
\begin{gathered}
k(\xi, x) \stackrel{\text { def }}{=} k_{\bar{I}}^{j \bar{J}}(\xi, x)=A_{\bar{I}}^{j \bar{J}}(\xi, x) \frac{\left(r_{\zeta^{j}}-r_{z^{j}}\right)}{\left(N_{0}^{a_{0}} S_{0}^{b_{0}}\right)(\zeta, z)}, \quad a_{0}+b_{0}=n, \quad b_{0}=q, \\
l(\xi, x) \stackrel{\text { def }}{=} l_{\bar{I} s}^{\alpha \beta \bar{J}}(\xi, x)=B_{\bar{I} s}^{\alpha \beta \bar{J}}(\xi, x) \frac{r_{\zeta^{\beta}}\left(r_{\zeta^{\alpha}}-r_{z^{\alpha}}\right)}{\left(\zeta^{n}-z^{n}\right)\left(N_{0}^{a_{0}-1} S_{0}^{b_{0}}\right)(\zeta, z)} .
\end{gathered}
$$

Recall that $\chi=\chi_{\sigma, \rho}$ is a smooth function, which is 1 on $D_{\rho(1-\sigma / 2)}$ and zero off $D_{\rho(1-\sigma / 4)}$, and $\|\chi\|_{\rho, a} \leq C_{a}(\rho \sigma)^{-a}$. On $D_{\rho}$, set

$$
\varphi_{0}=\chi \varphi, \quad \varphi_{1}=\varphi-\varphi_{0} .
$$

Assume that $M$ satisfies (2.1)-(2.2). Thus $\|\hat{r}\|_{\rho_{0}, 2}<1 / C_{0}$. Assume that $0<\rho \leq$ $\rho_{0} \leq 3$. Set $R=\left|z^{\prime}\right|^{2}+\hat{r}(x)$. Let $\pi_{s}$ be the projection from $\partial D_{\rho}$ into the subspace $\xi^{s}=0$. Since $\bar{D}_{\rho}$ is bounded and strictly convex, then $\pi_{s}$ is a 2-to-1 map from $\partial D_{\rho}$ onto $\pi_{s}\left(\partial D_{\rho}\right)=\pi\left(\bar{D}_{\rho}\right)$. Actually, $\pi_{s}$ sends $\pi_{s}^{-1}\left(\partial\left(\pi_{s}\left(D_{\rho}\right)\right)\right)$ one-to-one and onto $\partial\left(\pi_{s}\left(D_{\rho}\right)\right)$. Let $\operatorname{vol}\left(\pi_{s} D_{\rho}\right)$ be the volume of $\pi_{s}\left(D_{\rho}\right)$ calculated via the volumeform $d V^{s}$. Recall that $D_{\rho}$ is contained in $B_{2 \rho}$. If $f$ is a continuous function on $D_{\rho}$, then

$$
\begin{aligned}
\left|\int_{\partial D_{\rho}} f(\xi) d V^{s}(\xi)\right| & \leq 2 \operatorname{vol}\left(\pi_{s} D_{\rho}\right)\|f\|_{\rho, 0} \leq 2 \operatorname{vol}\left(B_{2 \rho} \cap \mathbb{R}^{2 n-2}\right)\|f\|_{\rho, 0} \\
& \leq C \rho^{2 n-2}\|f\|_{\rho, 0} .
\end{aligned}
$$

Since the projection of $\partial D_{\rho}$ in any coordinate hyperplane is contained in a ball of radius $2 \rho$, by the Fubini theorem one can verify that

$$
\operatorname{vol}\left(D_{\rho} \backslash D_{\left(1-\frac{1}{2} \sigma\right) \rho}\right) \leq(2 n-1) \cdot C \rho \sigma \cdot \rho^{2 n-2} \leq C^{\prime} \rho^{2 n-1} \sigma .
$$

Let $k \geq 0$ be an integer and $0 \leq \alpha<1$. Fix $\zeta \in M_{\rho} \backslash M_{\left(1-\frac{1}{2} \sigma\right) \rho}$ and vary $z \in M_{(1-\sigma) \rho}$. By Lemma 6.3, we have

$$
\left|r_{\zeta} \cdot(\zeta-z)\right| \geq C^{-1}(\rho \sigma)^{2}, \quad\left|r_{z} \cdot(\zeta-z)\right| \geq C^{-1}(\rho \sigma)^{2} .
$$

We also have $\left|r_{\zeta^{j}}-r_{z^{j}}\right| \leq C\|\zeta-z\| \leq C^{\prime}\left|r_{\zeta} \cdot(\zeta-z)\right|^{1 / 2}$. Hence

$$
\left|r_{\zeta^{j}}-r_{z^{j}}\right|\left|N_{0}\right|^{-a}\left|S_{0}\right|^{-b} \leq C\left|N_{0}\right|^{\frac{1}{2}-a-b} \leq C^{\prime}(\rho \sigma)^{1-2 a-2 b}
$$

if $a+b \geq 1 / 2$. Using $\left|f\left(x_{2}\right)-f\left(x_{1}\right)\right| \leq C\|f\|_{\rho, 1}\left|x_{2}-x_{1}\right| \leq C\|f\|_{\rho, 1}\left(\left|x_{2}\right|+\right.$ $\left.\left|x_{1}\right|\right)^{1-\alpha}\left|x_{2}-x_{1}\right|^{\alpha}$, we get

$$
\|f\|_{\rho, \alpha} \leq\|f\|_{\rho, 0}+C\|f\|_{\rho, 1} \rho^{1-\alpha} .
$$


Therefore, for $1 \leq j \leq n$ and $0<\alpha<1$,

$$
\left\|r_{\zeta^{j}}-r_{z^{j}}(\cdot)\right\|_{\rho, \alpha} \leq C \rho^{1-\alpha}, \quad\left|r_{\zeta^{\beta}}\right| \leq C \rho .
$$

Also for $x_{1}, x_{2} \in D_{\rho}$,

$$
\begin{aligned}
\left|\frac{1}{f\left(x_{2}\right)}-\frac{1}{f\left(x_{1}\right)}\right| & =\frac{\left|f\left(x_{1}\right)-f\left(x_{2}\right)\right|^{1-\alpha}}{\left|f\left(x_{2}\right) f\left(x_{1}\right)\right|}\left|f\left(x_{1}\right)-f\left(x_{2}\right)\right|^{\alpha} \\
& \leq C 2^{1-\alpha}\|1 / f\|_{\rho, 0}^{1+\alpha}\|f\|_{\rho, 1}^{\alpha}\left|x_{2}-x_{1}\right|^{\alpha} .
\end{aligned}
$$

Combining it with Hölder ratio $\left|1 / f^{a}\right|_{\rho, \alpha} \leq C_{a}\|1 / f\|_{\rho, 0}^{a-1}|1 / f|_{\rho, \alpha}$ for $a \geq 1$, we get

$$
\left\|1 / f^{a}\right\|_{\rho, \alpha} \leq\left\|1 / f^{a}\right\|_{\rho, 0}+C_{a}\|1 / f\|_{\rho, 0}^{a+\alpha}\|f\|_{\rho, 1}^{\alpha}, \quad a \geq 1 .
$$

Now, by (10.3)

$$
\left\|N_{0}(\zeta, \cdot)^{-a}\right\|_{(1-\sigma) \rho, \alpha}+\left\|S_{0}(\zeta, \cdot)^{-a}\right\|_{(1-\sigma) \rho, \alpha} \leq C(\rho \sigma)^{-2(a+\alpha)}, \quad a \geq 1 .
$$

Note that $A_{\bar{I}}^{j \bar{J}}$ has the form $\partial_{*}^{2} r$. By Proposition A.5, we have

$$
\left\|\partial_{*}^{2} r(\xi, \cdot)\right\|_{\rho, a} \cdot\left\|\partial_{*}^{2} r(\xi, \cdot)\right\|_{\rho, b} \leq C_{a, b} \rho^{-a-b}\|r\|_{\rho, 2+a+b} .
$$

We have

$$
\partial_{x}^{K} k(\xi, x)=\sum_{a+b+c+|L|=|K|} \sum_{|L|=0,1} \frac{\partial_{*}^{2+c} r(\xi, x) \partial_{x}^{L}\left(r_{\zeta^{j}}-r_{z^{j}}\right)}{\left(N_{0}^{a_{0}+a} S_{0}^{b_{0}+b}\right)(\zeta, z)},
$$

where $a_{0}+b_{0}=n$. Fix $\xi$ and vary $x$. For the summand, we estimate the $\mathcal{C}^{\alpha}$-norms of two terms in the numerator by (10.3)-(10.5), and use (10.7) for the reciprocals of two terms in the denominator. Set $|K|=k$ and $|L|=d$. Recall that $d=0$ or 1 . We get

$$
\begin{aligned}
\| & \frac{\partial_{*}^{2+c} r(\xi, \cdot) \partial_{x}^{L}\left(r_{\zeta^{j}}-r_{z^{j}}(\cdot)\right)}{\left(N_{0}^{a_{0}+a} S_{0}^{b_{0}+b}\right)(\zeta, \cdot)} \|_{(1-\sigma) \rho, \alpha} \\
\leq & \frac{C}{(\rho \sigma)^{2\left(a+a_{0}+b+b_{0}\right)}}\left\{(\rho \sigma)^{1-d} \rho^{-c-\alpha}\|r\|_{\rho, 2+c+\alpha}\right. \\
\quad & \left.\quad \rho^{(1-d)(1-\alpha)} \rho^{-c-d \alpha}\|r\|_{\rho, 2+c+d \alpha}+2(\rho \sigma)^{1-d}(\rho \sigma)^{-2 \alpha} \rho^{-c}\|r\|_{\rho, 2+c}\right\} .
\end{aligned}
$$

The worst term in terms of powers of $\rho, \sigma$ occurs when $a+b=k, c=d=0$. We see that

$$
\|k(\xi, \cdot)\|_{(1-\sigma) \rho, k+\alpha} \leq C_{k}(\rho \sigma)^{-s_{1}}\|r\|_{\rho, k+2+\alpha}, \quad \xi \in D_{\rho} \backslash D_{\left(1-\frac{1}{2} \sigma\right) \rho}
$$

with $s_{1}=2 n-1+2 k+2 \alpha$. 
To estimate the boundary term, by (4.2) we have

$$
\left|\zeta^{n}-z^{n}\right| \geq C^{-1} \rho^{2} \sigma
$$

for $z \in M_{(1-\sigma) \rho}$ and $\zeta \in \partial M_{\rho}$. Using (10.6), we get for $\zeta \in \partial M_{\rho}$

$$
\left\|\left(\zeta^{n}-z^{n}(\cdot)\right)^{-a}\right\|_{(1-\sigma) \rho, \alpha} \leq C\left(\rho^{2} \sigma\right)^{-a-\alpha}, \quad a \geq 1, \quad 0 \leq \alpha \leq 1 .
$$

Note that $B_{\bar{I} s}^{\alpha \beta \bar{J}}$ has the form $\partial_{*}^{2} r$. We have

$$
\partial_{x}^{K} l(\xi, x)=\sum_{a+b+d+c=|K|} \sum_{c_{1}+|L|=c} \sum_{|L|=0,1} \frac{\partial_{*}^{2+c_{1}} r \cdot r_{\zeta^{\beta}} \cdot \partial_{x}^{L}\left(r_{\zeta^{j}}-r_{z^{j}}\right)}{\left(\zeta^{n}-z^{n}\right)^{1+d}\left(N_{0}^{a_{0}-1+a} S_{0}^{b_{0}+b}\right)(\zeta, z)} .
$$

Set $|K|=k$ and $|L|=c_{2}$. We now estimate the $\mathcal{C}^{\alpha}$-norm in the $x$ variables. Fix $\zeta \in \partial D_{\rho}$. Using (10.3)-(10.5), (10.8) for three terms in the numerator and (10.7), (10.10)-(10.11) for the reciprocals of three terms in the denominator, we obtain

$$
\begin{gathered}
\|l(\xi, \cdot)\|_{(1-\sigma) \rho, k+\alpha} \leq C_{k} \sum_{a+b+d+c_{1}+c_{2}=k} \sum_{c_{2}=0,1}(\rho \sigma)^{-2\left(a_{0}+a+b_{0}+b+d\right)} \sigma^{1+d} \rho . \\
\cdot\left\{(\rho \sigma)^{1-c_{2}} \rho^{-c_{1}-\alpha}\|r\|_{\rho, 2+c_{1}+\alpha}+\rho^{\left(1-c_{2}\right)(1-\alpha)} \rho^{-c_{1}-c_{2} \alpha}\|r\|_{\rho, 2+c_{1}+c_{2} \alpha}\right. \\
\left.+\rho^{-c_{1}}(\rho \sigma)^{1-c_{2}}\left(\left(\rho^{2} \sigma\right)^{-\alpha}+2(\rho \sigma)^{-2 \alpha}\right)\|r\|_{\rho, 2+c_{1}}\right\} .
\end{gathered}
$$

The worst term in terms of powers of $\rho, \sigma$ occurs when $c_{1}=c_{2}=d=0, a+b=k$. This shows that for each $\zeta \in \bar{M}_{\rho} \backslash M_{\left(1-\frac{1}{2} \sigma\right) \rho}$, we have

$$
\|l(\xi, \cdot)\|_{(1-\sigma) \rho, k+\alpha} \leq C_{k}(\rho \sigma)^{-s_{2}}\|r\|_{\rho, k+2+\alpha}
$$

with $s_{2}=2(n+k-1+\alpha)$.

By (10.1) and (10.13), we estimate the boundary term by

$$
\left\|P_{1}^{\prime} \varphi\right\|_{(1-\sigma) \rho, k+\alpha} \leq C_{k} \rho^{2 n-2}(\rho \sigma)^{-s_{2}}\|r\|_{\rho, k+\alpha+2}\|\varphi\|_{\rho, 0} .
$$

Estimating the cutoff term by (10.9) and (10.2), we obtain

$$
\left\|P_{0}^{\prime} \varphi_{1}\right\|_{(1-\sigma) \rho, k+\alpha} \leq C_{a} \rho^{2 n-1-s_{1}} \sigma^{1-s_{1}}\|r\|_{\rho, k+2+\alpha}\|\varphi\|_{\rho, 0} .
$$

Define $s \stackrel{\text { def }}{=} \max \left\{s_{1}-1, s_{2}, k+\alpha\right\}$ and $s_{*} \stackrel{\text { def }}{=} \max \left\{s_{1}-2 n+1, s_{2}-2 n+2, k+\alpha-1\right\}$. Thus for $a=k+\alpha$, we get

$$
s=2(a+n-1), \quad s_{*}=2 a .
$$

Combining (9.2), (10.14)-(10.15), we get the following. 
Proposition 10.1. Let $n \geq 4$, and let $a \geq 0$ be a real number. Let $M: y^{n}=$ $\left|z^{\prime}\right|^{2}+\hat{r}\left(z^{\prime}, x^{n}\right)$ satisfy (2.2). Let $P^{\prime}$ be either of $P^{\prime}, Q^{\prime}$ in the homotopy formula $\varphi=\bar{\partial}_{M} P^{\prime} \varphi+Q^{\prime} \bar{\partial}_{M} \varphi$ on $M_{\rho}$. Assume that $0<\rho \leq \rho_{0} \leq 3$. Then for a tangential form $\varphi$

$$
\left\|P^{\prime} \varphi\right\|_{D_{(1-\sigma) \rho}, a} \leq C_{a} \rho^{-s_{*}} \sigma^{-s}\left(\|\varphi\|_{D_{\rho}, a}+\|\varphi\|_{D_{\rho}, 0}\|\hat{r}\|_{D_{\rho}, a+2}\right),
$$

where $0<\sigma<1, s, s_{*}$ are given by (10.16).

\section{Reduction of $\mathcal{C}^{k+\frac{1}{2}}$-estimates to $\mathcal{C}^{\frac{1}{2}}$-estimate. Summary}

We want to prove the following $\mathcal{C}^{k+\frac{1}{2}}$ estimates.

Proposition 11.1. Let $n \geq 4$, and let $k \geq 0$ be an integer. Let $M: y^{n}=\left|z^{\prime}\right|^{2}+$ $\hat{r}\left(z^{\prime}, x^{n}\right)$ satisfy (2.2). Let $P^{\prime}$ be one of $P^{\prime}, Q^{\prime}$ in the homotopy formula $\varphi=$ $\bar{\partial}_{M} P^{\prime} \varphi+Q^{\prime} \bar{\partial}_{M} \varphi$ on $M_{\rho}$. Then for $0<\rho \leq \rho_{0} \leq 3,0<\sigma<1$, and a tangential $(0, q)$ form $\varphi$

$$
\begin{gathered}
\left\|P^{\prime} \varphi\right\|_{D_{(1-\sigma) \rho}, k+\frac{1}{2}} \leq \frac{C_{k}}{\rho^{2 k+1} \sigma^{2 n+2 k-1}}\left(\|r\|_{D_{\rho}, \frac{5}{2}}\|\varphi\|_{D_{\rho}, k}+\|\hat{r}\|_{D_{\rho}, k+\frac{5}{2}}\|\varphi\|_{D_{\rho}, 0}\right), \\
\|P \varphi\|_{D_{(1-\sigma) \rho}, \frac{1}{2}} \leq C \rho^{-1} \sigma^{1-2 n}\|\varphi\|_{D_{\rho}, 0}, \quad q=1 .
\end{gathered}
$$

Proof. Fix a positive integer $k$. To estimate the $\mathcal{C}^{k+\frac{1}{2}}$-norm of $P^{\prime} \varphi=\left(P_{0}^{\prime}+P_{1}^{\prime}\right) \varphi$, we first recall estimates (10.14) and (10.15) for the boundary and cutoff terms

$$
\left\|\left(P_{0}^{\prime} \varphi_{1}, P_{1}^{\prime} \varphi\right)\right\|_{(1-\sigma) \rho, k+\frac{1}{2}} \leq C_{a} \rho^{-S_{*}} \sigma^{-s}\|r\|_{\rho, k+\frac{5}{2}}\|\varphi\|_{\rho, 0}
$$

Here $s=2 n+2 k-1$ and $s_{*}=2 k+1$ are computed by (10.16) for $a=k+\frac{1}{2}$. It remains to estimate the $\mathcal{C}^{k+\frac{1}{2}}$-norm of $P_{0}^{\prime} \varphi_{1}$, where $\varphi_{1}=\chi \varphi$ and $\chi$ has compact support in $D_{\rho}$ and $\|\chi\|_{\rho, a} \leq C_{a}(\rho \sigma)^{-a}$. The proof will be completed later. For the rest of proof, we reduce it to the special case of $k=0$.

The second formula (8.12) says that the coefficients of $\partial^{k} P_{0}^{\prime} \varphi_{0}$ are sums of

$$
\mathcal{K} u(x)=\int_{D_{\rho}} u(\xi, x) k(\xi, x) d V
$$

with functions $u(\xi, x)$ and kernels $k(\xi, x)$ of the form

$$
\begin{gathered}
u(\xi, x)=\partial^{E} \chi(\xi) \partial^{F} \varphi \bar{J}(\xi) \partial_{*}^{2+l} r(\xi, x), \quad|E|+|F|+l=k, \\
k(\xi, x) \stackrel{\text { def }}{=} k_{a b}^{I}(\zeta, z)=\frac{\left(\zeta^{\prime}-z^{\prime}, \bar{\zeta}^{\prime}-z^{\prime}, \operatorname{Im}\left(r_{z} \cdot(\zeta-z)\right)\right)^{I}}{\left(r_{\zeta} \cdot(\zeta-z)\right)^{a}\left(r_{z} \cdot(\zeta-z)\right)^{b}}, \quad \zeta, z \in M_{\rho} .
\end{gathered}
$$


Moreover, by (8.11), the non-negative integers $a, b, I=\left(i_{1}, \ldots, i_{2 n-1}\right)$ satisfy

$$
|I|-2 a-2 b \geq 1-2 n, \quad|I| \leq 2 k+1, \quad a+b \leq n+k .
$$

By (9.8) in Proposition 9.2, we have

$$
\|u(\xi, \cdot)\|_{\rho, \frac{1}{2}} \leq C_{k}(\rho \sigma)^{-k-\frac{1}{2}}\left(\|\varphi\|_{\rho, k}\|r\|_{\rho, \frac{5}{2}}+\|\varphi\|_{\rho, 0}\|\hat{r}\|_{\rho, k+\frac{5}{2}}\right) .
$$

In Section 12 we will prove that if $u \in L^{\infty}\left(D_{\rho} \times D_{\rho}\right)$ then

$$
\|\mathcal{K} u\|_{D_{\rho}, 1 / 2} \leq C \sup _{\xi \in D_{\rho}}\|u(\xi, \cdot)\|_{D_{\rho}, 1 / 2} .
$$

Combining it with (11.1) and (11.6) yields the first estimate in the proposition.

We now consider the case that $\varphi$ is a tangential $(0,1)$-form. We return to (3.10)-(3.11) and look at a special property of the kernels of $P^{\prime} \varphi$. Recall that in this case

$$
\begin{gathered}
P_{0}^{\prime} \varphi(x)=\sum_{1 \leq \gamma<n} \sum_{1 \leq j \leq n} \int_{D_{\rho}} A^{j \bar{\gamma}}(\xi, x) \frac{\varphi_{\bar{\gamma}}(\xi)\left(r_{\zeta^{j}}-r_{z^{j}}\right)}{\left(N_{0}^{n-1} S_{0}\right)(\zeta, z)} d V(\xi), \\
P_{1}^{\prime} \varphi(x)=\sum_{1 \leq \alpha, \beta, \gamma<n} \sum_{1 \leq s<2 n} \int_{\partial D_{\rho}} \frac{B_{s}^{\alpha \beta \bar{\gamma}}(\xi, x) \varphi_{\bar{\gamma}}(\xi)\left(r_{\zeta^{\alpha}}-r_{z^{\alpha}}\right) r_{\zeta^{\beta}}}{\left(\zeta^{n}-z^{n}\right)\left(N_{0}^{n-2} S_{0}\right)(\zeta, z)} d V^{s}(\xi) .
\end{gathered}
$$

Here $\zeta, z \in M_{\rho}$. Also, $A^{j \bar{\gamma}}$ and $B_{s}^{\alpha \beta \bar{\gamma}}$ are polynomials in $r_{\zeta}, r_{\bar{\zeta}}, r_{\zeta \bar{\zeta}}$. In particular, they are independent of $z$. Moreover, in the kernels there are only the first-order derivatives $r_{z^{j}}$ in the $z$ variable. Then all norms of $r$ in (10.12)-(10.14), in which $k=0$, can be replaced by $\|r\|_{\rho, 2}<C$ and the estimate (10.14) for the boundary term becomes

$$
\left\|P_{1}^{\prime} \varphi\right\|_{(1-\sigma) \rho, \alpha} \leq C \rho^{-2 \alpha} \sigma^{2-2 n-2 \alpha}\|\varphi\|_{\rho, 0}, \quad 0 \leq \alpha \leq 1 .
$$

Absorb $A_{I}^{j \bar{\gamma}}(\xi)$ into $\varphi_{\bar{\gamma}}(\xi)$. With $\zeta, z \in M_{\rho}$ the kernels of interior integral $P_{0}^{\prime} \varphi$ have the form

$$
k(\xi, x)=\frac{r_{\zeta_{j}}-r_{z^{j}}}{\left(r_{\zeta} \cdot(\zeta-z)\right)^{a}\left(r_{z} \cdot(\zeta-z)\right)^{b}}, \quad a+b=n .
$$

We will show that (11.7) holds for this new kernel. Applying it to

$$
u(\xi, x)=A^{j \bar{\gamma}}(\xi) \varphi_{\bar{\gamma}}(\xi), \quad \zeta \in M
$$

we obtain $\left\|P_{0}^{\prime} \varphi_{0,1}\right\|_{\rho, 1 / 2} \leq C\left\|\varphi_{0,1}\right\|_{\rho, 0}$. This shows the second estimate of the proposition.

The proof of Proposition 11.1 is thus complete, by assuming (11.7) in which $\mathcal{K} u$, given by (11.2), has a kernel of the form (11.4)-(11.5) or (11.8). 
We want to unify the two kernels (11.4), which satisfies (11.5), and (11.8). For (11.4), we write

$$
\operatorname{Im}\left(r_{z} \cdot(\zeta-z)\right)=\frac{1}{2 i} r_{z} \cdot(\zeta-z)-\frac{1}{2 i} \overline{r_{z} \cdot(\zeta-z)}
$$

Then kernel (11.4) is a linear combination of

$$
k(\xi, x) \stackrel{\text { def }}{=} \frac{\left(\zeta-z, \overline{\zeta-z}, r_{\zeta}-r_{z}\right)^{I}}{\left(r_{\zeta} \cdot(\zeta-z)\right)^{a}\left(r_{z} \cdot(\zeta-z)\right)^{b} \overline{\left(r_{\zeta} \cdot(\zeta-z)\right)^{c}} \overline{\left(r_{z} \cdot(\zeta-z)\right)^{d}}},
$$

where $\zeta, z \in M, a, b, c, d$ are now possibly negative integers, $I$ is a (3n)-tuple of nonnegative integers, and

$$
|I|-2(a+b+c+d) \geq 1-2 n,|I| \leq 2 k+1,|a|+|b|+|c|+|d| \leq n+3 k+2 .
$$

Indeed, the $I=\left(i_{1}, \ldots, i_{2 n-1}\right)$ in (11.5) is a multi-index of nonnegative integers. Hence, (11.5) and $i_{2 n-1} \geq 0$ implies (11.10). Obviously, (11.8) is of the form (11.9). In Section 12, for the new kernel (11.9) with the condition (11.10) we will prove (11.7), i.e.

$$
\left\|\int_{D_{\rho}} u(\xi, \cdot) k(\xi, \cdot) d V(\xi)\right\|_{D_{\rho}, 1 / 2} \leq C \sup _{\xi \in D_{\rho}}\|u(\xi, \cdot)\|_{D_{\rho}, 1 / 2}
$$

where $u \in L^{\infty}\left(D_{\rho} \times D_{\rho}\right)$.

Summary. We would like to summarize our observations on $\mathcal{C}^{k+\frac{1}{2}}$-estimates to explain how the general estimate (11.11) gives us an actual $\frac{1}{2}$-gain in special cases.

a) $k \geq 1$ and $q \geq 1$. In our applications of (11.11) to the $\mathcal{C}^{k+\frac{1}{2}}$-estimates, $u(\xi, x)$, arising from the $k$-th order derivatives of $P_{0}^{\prime} \varphi$ after applying the approximate Heisenberg transformation, is of the form $\partial^{k-j} \varphi_{\bar{J}}(\xi) \partial_{*}^{j+2} r(\xi, x)$ where $\varphi$ has compact support in $D_{\rho}$; see the second formula of the derivatives in Section 8. In particular, the $\mathcal{C}^{\frac{1}{2}}$-norm of $u(\xi, x)$ in $x$ variables involves only $\|\varphi\|_{\rho, k}$ (and $\left.\|r\|_{\rho, k+\frac{5}{2}}\right)$. Thus the estimate for $\left\|P_{0}^{\prime} \varphi\right\|_{\rho, k+\frac{1}{2}}$ gains $\frac{1}{2}$ in Hölder exponent from $\|\varphi\|_{\rho, k}$ at the expense of two extra derivatives in $\|r\|_{\rho, k+\frac{5}{2}}$.

b) $k=0$ and $q=1$. Apply $(11.11)$ to $(0,1)$ forms. In this case, we will not need to differentiate the kernels and apply cutoff. Both simplify the estimate considerably. However, it is crucial that when (11.11) is applied, $u(\xi, x)$ has the form $A^{j \bar{\gamma}}(\xi) \varphi_{\bar{\gamma}}(\xi)$; in particular, it is independent of $x$. Therefore, the $\frac{1}{2}$-estimate of $u$ in the $x$-variables and hence $\frac{1}{2}$-estimate of $P_{0}^{\prime} \varphi$ only require $r \in \mathcal{C}^{2}$.

c) While the $\frac{1}{2}$-estimate of $P_{0}^{\prime} \varphi$ is on the original domain (Proposition 12.1 below), the estimate for the boundary term $P_{1}^{\prime} \varphi$, which only requires $r \in \mathcal{C}^{2}$ for the same reason as in $b$ ), is on shrinking domains. 


\section{2. $\frac{1}{2}$-estimate}

We will derive the $\frac{1}{2}$-estimate, by modifying a standard argument for Hölder estimates. This will complete the proof of Proposition 11.1 via (11.6) and (11.11). We restate the latter as the following.

Proposition 12.1. Let $M$ be a real hypersurface of class $\mathcal{C}^{2}$ and satisfy (2.2). Assume that $0<\rho \leq \rho_{0} \leq 3$. Let $a, b, c, d$ be (possibly negative) integers and I be $a$ (3n)-tuple of nonnegative integers. Suppose that $|I|-2(a+b+c+d) \geq 1-2 n$. Let

$$
\begin{gathered}
k(\xi, x)=\frac{\left(\zeta-z, \overline{\zeta-z}, r_{\zeta}-r_{z}\right)^{I}}{\left(r_{\zeta} \cdot(\zeta-z)\right)^{a}\left(r_{z} \cdot(\zeta-z)\right)^{b} \overline{\left(r_{\zeta} \cdot(\zeta-z)\right)^{c}} \overline{\left(r_{z} \cdot(\zeta-z)\right)^{d}}}, \quad \zeta, z \in M, \\
\mathcal{K} u(x)=\int_{M_{\rho}} u(\xi, x) k(\xi, x) d V .
\end{gathered}
$$

Then for $u \in L^{\infty}\left(D_{\rho} \times D_{\rho}\right)$,

$$
\|\mathcal{K} u\|_{D_{\rho}, \frac{1}{2}} \leq C\left(\rho \sup _{\xi \in D_{\rho}}\|u(\xi, \cdot)\|_{D_{\rho}, \frac{1}{2}}+\sup _{\xi \in D_{\rho}}\|u(\xi, \cdot)\|_{D_{\rho}, 0}\right) .
$$

Proof. Recall that $D_{\rho}$ is convex. It will be convenient to regard $K u$ as a function on $M$. The $\frac{1}{2}$-Hölder ratios on $M_{\rho}$ and $\pi M_{\rho}$ are equivalent, since $\left|\pi\left(z_{2}-z_{1}\right)\right| \geq$ $\left|z_{2}-z_{1}\right| / C$ for $z_{1}, z_{2} \in M_{\rho}$. By abuse of notation, we write $u(\xi, x)$ as $u(\zeta, z)$ with $\zeta, z \in M$ and apply the same change of notation to $k(\xi, x), \mathcal{K} u(x)$.

Fix $z_{1}, z_{2}$ in $M_{\rho}$. We have

$$
\begin{aligned}
\mathcal{K} u\left(z_{2}\right)-\mathcal{K} u\left(z_{1}\right)= & \int_{M_{\rho}} u\left(\zeta, z_{2}\right)\left(k\left(\zeta, z_{2}\right)-k\left(\zeta, z_{1}\right)\right) d V \\
& +\int_{M_{\rho}}\left(u\left(\zeta, z_{2}\right)-u\left(\zeta, z_{1}\right)\right) k\left(\zeta, z_{1}\right) d V=\mathcal{I}+\mathcal{I}^{\prime}
\end{aligned}
$$

To estimate $\mathcal{I}^{\prime}$, we need to estimate $\int_{M_{\rho}}\left|k\left(\xi, z_{1}\right)\right| d V(\xi)$. So we apply the approximate Heisenberg transformation $\zeta_{*}=\psi_{z_{1}}(\zeta$ ). (However, we want to refrain from use of Taylor remainder expansions, such as (8.4)-(8.5), for the kernel. This avoids the requirement of $r \in \mathcal{C}^{5 / 2}$.) Let $\zeta \in M_{\rho}$. Using the fundamental theorem of calculus, we get $\eta^{n}-y_{1}^{n}=A\left(\xi, x_{1}\right) \cdot\left(\xi-x_{1}\right)$ with $|A|<C$. By (6.4), we have $\xi^{n}-x_{1}^{n}=p\left(\xi, x_{1}\right) \cdot \xi_{*}$ with $|p|<C$. Therefore,

$$
\left|\zeta-z_{1}\right| \leq C\left|\xi_{*}\right|
$$

By Lemma 6.1, we have

$$
\left|r_{\zeta}-r_{z_{1}}\right| \leq C\left|\xi_{*}\right|, \quad 1 / C \leq \frac{\left|r_{z_{1}} \cdot\left(\zeta-z_{1}\right)\right|}{\left.|| \zeta_{*}^{\prime}\right|^{2}+i \xi_{*}^{n} \mid} \leq C, \quad 1 / C \leq \frac{\left|r_{\zeta} \cdot\left(\zeta-z_{1}\right)\right|}{\left.|| \zeta_{*}^{\prime}\right|^{2}+i \xi_{*}^{n} \mid} \leq C
$$


Therefore, for $\zeta \in M_{\rho}$

$$
\left|k\left(\zeta, z_{1}\right)\right| \leq \frac{C\left|\xi_{*}\right|^{|I|}}{\left.|| \zeta_{*}^{\prime}\right|^{2}+\left.i \xi_{*}^{n}\right|^{a+b+c+d}} \leq \sum_{|J|=|I|} \frac{C\left|\xi_{*}^{J}\right|}{\left.|| \zeta_{*}^{\prime}\right|^{2}+\left.i \xi_{*}^{n}\right|^{a+b+c+d}}
$$

By Lemma 5.2, $\pi\left(\psi_{z_{1}}\left(M_{\rho}\right)\right) \subset B_{9 \rho}$. Applying Lemma 7.1 with $\rho_{1}=\rho_{0}=9 \rho$ and $\beta \geq 0$, we get

$$
\int_{M_{\rho}}\left|k\left(\zeta, z_{1}\right)\right| d V \leq \sum_{|J|=|I|} \int_{B_{9 \rho}} \frac{C\left|\xi_{*}^{J}\right|}{\left.\left.\left|\xi_{*}^{n}+i\right| \zeta_{*}^{\prime}\right|^{2}\right|^{a+b+c+d}} d V \leq C^{\prime} \rho .
$$

Since $u(\zeta, z)$ is of class $\mathcal{C}^{1 / 2}$ in the $z$-variable, one gets

$$
|\mathcal{I}| \leq C\left|z_{2}-z_{1}\right|^{1 / 2} \rho \sup _{\zeta \in D_{\rho}}\|u(\zeta, \cdot)\|_{\rho, 1 / 2}
$$

To estimate the integral $\mathcal{I}^{\prime}$, it suffices to show that

$$
\int_{M_{\rho}}\left|k\left(\zeta, z_{2}\right)-k\left(\zeta, z_{1}\right)\right| d V \leq C \delta^{1 / 2}, \quad \delta=\left|z_{2}-z_{1}\right| .
$$

As mentioned in the introduction we decompose $M_{\rho}$ into a cylinder and its complement. Consider the cylinder $M_{\rho} \cap\left\{\left|\zeta^{\prime}-z_{1}^{\prime}\right|<\rho_{1}\right\}$, where $\rho_{1}=\min \left(9 \rho, C_{*} \mid z_{2}-\right.$ $\left.z_{1}\right|^{\frac{1}{2}}$ ) with $C_{*}>1$ to be determined. Notice that the radius of cylinder is about $\left|z_{2}-z_{1}\right|^{1 / 2}$, which is much large than $\left|z_{2}-z_{1}\right|$, the Euclidean distance between $z_{1}, z_{2}$.

We have

$$
\begin{gathered}
\int_{M_{\rho}}\left|k\left(\zeta, z_{2}\right)-k\left(\zeta, z_{1}\right)\right| d V \leq \mathcal{I}_{1}+\mathcal{I}_{2}+\mathcal{I}_{3}, \\
\mathcal{I}_{1}=\int_{M_{\rho} \cap\left\{\left|\zeta^{\prime}-z_{1}^{\prime}\right|<\rho_{1}\right\}}\left|k\left(\zeta, z_{1}\right)\right| d V, \quad \mathcal{I}_{2}=\int_{M_{\rho} \cap\left\{\left|\zeta^{\prime}-z_{1}^{\prime}\right|<\rho_{1}\right\}}\left|k\left(\zeta, z_{2}\right)\right| d V, \\
\mathcal{I}_{3}=\int_{M_{\rho} \cap\left\{\left|\zeta^{\prime}-z_{1}^{\prime}\right| \geq \rho_{1}\right\}}\left|k\left(\zeta, z_{2}\right)-k\left(\zeta, z_{1}\right)\right| d V .
\end{gathered}
$$

By an analogy of (12.1), we have

$$
\mathcal{I}_{1} \leq \sum_{|J|=|I|} C \int_{\left|\zeta_{*}^{\prime}\right|<\rho_{1},\left|\zeta_{*}\right|<9 \rho} \frac{\left|\xi_{*}^{J}\right|}{\left.\left.\left|\xi_{*}^{n}+i\right| \zeta_{*}^{\prime}\right|^{2}\right|^{a+b+c+d}} d V
$$

By Lemma 7.1 with $\beta \geq 0$ and $\rho_{0}=9 \rho$ and $\rho_{1}=\min \left(9 \rho, C_{*}\left|z_{2}-z_{1}\right|^{\frac{1}{2}}\right)$, we get $\mathcal{I}_{1} \leq C \rho_{1} \leq C C_{*}\left|z_{2}-z_{1}\right|^{1 / 2}$. Note that

$$
M_{\rho} \cap\left\{\left|\zeta^{\prime}-z_{1}^{\prime}\right|<\rho_{1}\right\} \subset M_{\rho} \cap\left\{\left|\zeta^{\prime}-z_{2}^{\prime}\right|<\rho_{1}+\left|z_{2}-z_{1}\right|\right\} .
$$

Applying the estimate for $\mathcal{I}_{1}$, we get $\mathcal{I}_{2} \leq C\left(\rho_{1}+\left|z_{2}-z_{1}\right|\right) \leq C^{\prime} C_{*}\left|z_{2}-z_{1}\right|^{1 / 2}$. 
With $C_{*}>1$ to be determined, we have $\mathcal{I}_{3}=0$ if $\rho_{1}=9 \rho$. Therefore, we may assume that $\rho_{1}=C_{*}\left|z_{2}-z_{1}\right|^{\frac{1}{2}}$.

To estimate $\mathcal{I}_{3}$, we ignore any non-isotropic distance and connect $\left(z_{1}^{\prime}, x_{1}^{n}\right)$ and $\left(z_{2}^{\prime}, x_{2}^{n}\right)$ by a line segment in the convex domain $D_{\rho}$. Let $z(t)$ be the graph of the line segment in $M$. Let $\zeta$ be a point of $M_{\rho}$ which is not in the cylinder. Then

$$
\left|\zeta_{*}^{\prime}\right|=\left|\zeta^{\prime}-z_{1}^{\prime}\right| \geq \rho_{1}=C_{*}\left|z_{2}-z_{1}\right|^{1 / 2} .
$$

We have

$$
\begin{gathered}
\left|r_{z(t)} \cdot(\zeta-z(t))-r_{z_{1}} \cdot\left(\zeta-z_{1}\right)\right| \leq C_{0}\left|z_{2}-z_{1}\right|, \\
\left|r_{z_{1}} \cdot\left(\zeta-z_{1}\right)\right| \geq C_{1}\left|\zeta-z_{1}\right|^{2} \geq C_{1}\left|\zeta^{\prime}-z_{1}^{\prime}\right|^{2} \geq C_{1} C_{*}^{2}\left|z_{2}-z_{1}\right|,
\end{gathered}
$$

where the second inequality comes from Lemma 6.3. We now fix $C_{*}>1$ such that $C_{1} C_{*}^{2}>2 C_{0}$. (As remarked earlier, if $C_{*}\left|z_{2}-z_{1}\right|^{\frac{1}{2}}>9 \rho$, we already have $\mathcal{I}_{3}=0$.) We obtain

$$
\left|r_{z_{1}} \cdot\left(\zeta-z_{1}\right)\right| / 2 \leq\left|r_{z(t)} \cdot(\zeta-z(t))\right| \leq 2\left|r_{z_{1}} \cdot\left(\zeta-z_{1}\right)\right| .
$$

Recall that $\zeta_{*}=\psi_{z_{1}}(\zeta)$ and $\left.C^{-1}|| \zeta_{*}^{\prime}\right|^{2}+i \xi_{*}^{n}|\leq| r_{z_{1}} \cdot\left(\zeta-z_{1}\right)|\leq C|\left|\zeta_{*}^{\prime}\right|^{2}+i \xi_{*}^{n} \mid$. Using $\left|r_{z(t)} \cdot(\zeta-z(t))\right| / C \leq\left|r_{\zeta} \cdot(\zeta-z(t))\right| \leq C\left|r_{z(t)} \cdot(\zeta-z(t))\right|$, we get

$$
\begin{gathered}
\left.C^{-1}|| \zeta_{*}^{\prime}\right|^{2}+i \xi_{*}^{n}|\leq| r_{\zeta} \cdot(\zeta-z(t))|\leq C|\left|\zeta_{*}^{\prime}\right|^{2}+i \xi_{*}^{n} \mid, \\
\left.C^{-1}|| \zeta_{*}^{\prime}\right|^{2}+i \xi_{*}^{n}|\leq| r_{z(t)} \cdot(\zeta-z(t))|\leq C|\left|\zeta_{*}^{\prime}\right|^{2}+i \xi_{*}^{n} \mid .
\end{gathered}
$$

Write $k(\zeta, z)=\frac{p(\zeta, z)}{q(\zeta, z)}$ with $p(\zeta, z)=\left(\zeta^{\prime}-z^{\prime}, \overline{\zeta^{\prime}-z^{\prime}}, r_{\zeta}-r_{z}\right)^{I}$ and

$$
q(\zeta, z)=\left(r_{\zeta} \cdot(\zeta-z)\right)^{a}\left(r_{z} \cdot(\zeta-z)\right)^{b} \overline{\left(r_{\zeta} \cdot(\zeta-z)\right)^{c}} \overline{\left(r_{z} \cdot(\zeta-z)\right)^{d}} .
$$

For $\zeta \in M_{\rho}$ and $C_{*}>1$ we have

$$
\begin{aligned}
|\zeta-z(t)| & \leq\left|\zeta-z_{1}\right|+\left|z(t)-z_{1}\right| \leq\left|\zeta-z_{1}\right|+C\left|z_{2}-z_{1}\right| \\
& \leq\left|\zeta-z_{1}\right|+C C_{*}^{-2}\left|\zeta^{\prime}-z_{1}^{\prime}\right|^{2} \leq C^{\prime}\left|\zeta-z_{1}\right| .
\end{aligned}
$$

By (6.4), $\left|\xi^{n}-x_{1}^{n}\right| \leq C\left|\xi_{*}\right|$. Thus $\left|\zeta-z_{1}\right| \leq C\left|\xi_{*}\right|$. Therefore, $|\zeta-z(t)| \leq C\left|\xi_{*}\right|$ and

$$
|p(\zeta, z(t))| \leq C|\zeta-z(t)|^{|I|} \leq\left. C^{\prime}\left|\xi_{*}\right|\right|^{|I|} .
$$

By (12.2)-(12.3), we have

$$
\left|\{q(\zeta, z(t))\}^{-1}\right| \leq \frac{C}{\left.|| \zeta_{*}^{\prime}\right|^{2}+\left.i \xi_{*}^{n}\right|^{a+b+c+d}} .
$$


It is easy to see that $\left|z^{\prime}(t)\right| \leq C\left|x_{2}-x_{1}\right| \leq C\left|z_{2}-z_{1}\right|$. Then $\left|\partial_{t} q(\zeta, z(t))^{-1}\right|$ does not exceed the sum of

$$
\frac{C\left|z_{2}-z_{1}\right|}{\left|\left(r_{\zeta} \cdot(\zeta-z(t))\right)^{a^{\prime}}\left(r_{z(t)} \cdot(\zeta-z(t))\right)^{b^{\prime}} \overline{\left(r_{\zeta} \cdot(\zeta-z(t))\right)^{c^{\prime}}} \overline{\left(r_{z(t)} \cdot(\zeta-z(t))\right)^{d^{\prime}}}\right|},
$$

where $a^{\prime}+b^{\prime}+c^{\prime}+d^{\prime}=a+b+c+d+1$. Applying the product rule, we get

$$
\left|\partial_{t} p(\zeta, z(t))\right| \leq C\left|z_{2}-z_{1}\right| \sum_{\left|I^{\prime}\right|=|I|-1}\left|\left(\zeta-z(t), \overline{\zeta-z(t)}, r_{\zeta}-r_{z(t)}\right)^{I^{\prime}}\right| .
$$

Therefore,

$\left|\partial_{t} p(\zeta, z(t))\right| \leq C\left|z_{2}-z_{1}\right|\left|\left(\zeta_{*}^{\prime}, \xi_{*}^{n}\right)\right|^{|I|-1},\left|\partial_{t} \frac{1}{q(\zeta, z(t))}\right| \leq \frac{C\left|z_{2}-z_{1}\right|}{\left.|| \zeta_{*}^{\prime}\right|^{2}+\left.i \xi_{*}^{n}\right|^{a+b+c+d+1}}$.

By the mean-value-theorem, we get for $\zeta \in M_{\rho} \cap\left\{\left|\zeta^{\prime}-z^{\prime}\right|>\rho_{1}\right\}$

$$
\begin{aligned}
\left|k\left(\zeta, z_{2}\right)-k\left(\zeta, z_{1}\right)\right| & \leq C\left|z_{2}-z_{1}\right|\left\{\frac{\left|\xi_{*}\right|^{|I|}}{\left.|| \zeta_{*}^{\prime}\right|^{2}+\left.i \xi_{*}^{n}\right|^{a+b+c+d+1}}+\frac{\left|\xi_{*}\right|^{|I|-1}}{\left.|| \zeta_{*}^{\prime}\right|^{2}+\left.i \xi_{*}^{n}\right|^{a+b+c+d}}\right\} \\
& \leq C^{\prime}\left|z_{2}-z_{1}\right| \frac{\left|\xi_{*}\right|^{|I|}}{\|\left.\zeta_{*}^{\prime}\right|^{2}+\left.i \xi_{*}^{n}\right|^{a+b+c+d+1}} \\
& \leq \sum_{|J|=|I|} \frac{C^{\prime}\left|z_{2}-z_{1}\right|\left|\xi_{*}^{J}\right|}{\left.|| \zeta_{*}^{\prime}\right|^{2}+\left.i \xi_{*}^{n}\right|^{a+b+c+d+1}} .
\end{aligned}
$$

Applying Lemma 7.1 with $\rho_{1}=C_{*}\left|z_{2}-z_{1}\right|^{1 / 2}<9 \rho=\rho_{0}$ and $\beta \geq-2$, we get

$$
\mathcal{I}_{3} \leq C\left|z_{2}-z_{1}\right| \rho_{1}^{-1} \leq C\left|z_{2}-z_{1}\right|^{1 / 2} .
$$

\section{Proof of Theorem 1.1}

We now prove Theorem 1.1, following a KAM argument in [5]. We restate the theorem.

Theorem. Let $M: r=0$ be a strongly pseudoconvex real hypersurface of class $\mathcal{C}^{2}$ in $\mathbb{C}^{n}$ with $n \geq 4$. Let $\omega$ be a continuous $r \times r$ matrix of $(0,1)$-forms on $M$ satisfying the integrability condition $\bar{\partial}_{b} \omega=\omega \wedge \omega \bmod \bar{\partial} r$. Near each point of $M$ there exists a non-singular matrix $A \in \mathcal{C}^{1 / 2}(M)$ such that $\bar{\partial}_{b} A=-A \omega \bmod \bar{\partial} r$. Moreover, if a is a positive real number, $M$ is of class $\mathcal{C}^{a+2}$ and $\omega \in \mathcal{C}^{a}(M)$, there is a solution $A \in \mathcal{C}^{a}(M)$; if $k$ is a positive integer, $\omega \in \mathcal{C}^{k}$ and $M \in \mathcal{C}^{k+\frac{5}{2}}$, there is a solution $A \in \mathcal{C}^{k+\frac{1}{2}}(M)$.

Note that not all solutions have the same regularity. If $u$ is a continuous CR function vanishing nowhere on $M$, then $u A$ is still a solution. 
Non-isotropic dilations. The non-isotropic dilation $T_{\delta}: z \rightarrow\left(\sqrt{\delta} z^{\prime}, \delta z^{n}\right)$ with $\delta>0$ does not preserve the real hypersurface $M$. However, it is obvious that it sends $M^{\delta}=T_{\delta}^{-1} M$ onto $M$. By abuse of notation, we will denote $T_{\delta}$ its restriction to $\mathbb{C}^{n-1} \times \mathbb{R}$.

We want to show that $M$ can be put in the form (2.1)-(2.2) after a second order normalization and a non-isotropic dilation.

Let $M$ be a graph $y^{n}=\left|z^{\prime}\right|^{2}+\hat{r}\left(z^{\prime}, x^{n}\right)$ over $D$. Recall that $M_{\rho}=M \cap$ $\left\{\left(x^{n}\right)^{2}+y^{n}<\rho^{2}\right\}$ and

$$
D_{\rho}=\pi\left(M_{\rho}\right)=\left\{\left(z^{\prime}, x^{n}\right) \in D:\left|z^{\prime}\right|^{2}+\left|x^{n}\right|^{2}+\hat{r}\left(z^{\prime}, x^{n}\right)<\rho^{2}\right\} .
$$

Set $r(z)=-y^{n}+\hat{r}\left(z^{\prime}, x^{n}\right)$. Define

$$
\hat{r}^{\delta}(z)=\delta^{-2} \hat{r}\left(\delta z^{\prime}, \delta^{2} x^{n}\right), \quad r^{\delta}(z)=\delta^{-2} r\left(\delta z^{\prime}, \delta^{2} x^{n}\right) .
$$

Then $M^{\delta}=T_{\delta}^{-1} M$ is the graph over $D^{\delta}=T_{\delta}^{-1} D$, given by

$$
y^{n}=\left|z^{\prime}\right|^{2}+\hat{r}^{\delta}\left(z^{\prime}, x^{n}\right) \text {. }
$$

For $0<\delta<1$, we have

$$
M_{\rho}^{\delta}=M^{\delta} \cap\left\{\left|x^{n}\right|^{2}+y^{n}<\rho^{2}\right\} \subset M^{\delta} \cap\left\{\delta^{2}\left|x^{n}\right|^{2}+y^{n}<\rho^{2}\right\} .
$$

So $D_{\rho}^{\delta} \stackrel{\text { def }}{=} \pi\left(M_{\rho}^{\delta}\right) \subset T_{\delta}^{-1} D_{\rho}$ for $0<\delta<1$.

In Theorem 1.1, we need a local solution $A$. By a change of local holomorphic coordinates, we may assume that $\hat{r}(0)=\partial \hat{r}(0)=\partial^{2} \hat{r}(0)=0$. Therefore

$$
\left\|\hat{r}^{\delta}\right\|_{D_{1}^{\delta}, 2}<\epsilon, \quad \overline{D_{1}^{\delta}} \subset D^{\delta},
$$

if $\delta$ is sufficiently small.

Integrability conditions. We will find our solution through a sequence of frame changes. We also need a small norm of initial $\omega$ via dilation. Therefore, we need to verify that the integrability condition is preserved under dilation and frame changes.

Recall that for $\varphi=\sum_{|I|=q} \varphi_{\bar{I}} d{\overline{z^{\prime}}}^{I}$, we define $\bar{\partial}_{M} \varphi=\sum_{|I|=q, 1 \leq \alpha<n} X_{\bar{\alpha}} \varphi_{\bar{I}} d \overline{z^{\alpha}} \wedge$ $d{\overline{z^{\prime}}}^{I}$ for $X_{\bar{\alpha}}=\partial_{z^{\alpha}}-r_{z^{\alpha}} / r_{z^{n}} \partial_{z^{n}}$. A direct computation shows that $\delta d T_{\delta}^{-1} X_{\bar{\alpha}}=$ $X_{\bar{\alpha}}^{\delta} \equiv \partial_{z^{\alpha}}-r \bar{z}_{z^{\alpha}}^{\delta} / r r_{z^{n}} \partial_{z^{n}}$. Thus, $T_{\delta}^{*} \bar{\partial}_{M}=\bar{\partial}_{M^{\delta}} T_{\delta}^{*}$. This shows that the formal integrability condition is invariant under dilation, i.e. $\bar{\partial}_{M^{\delta}} \omega^{\delta}=\omega^{\delta} \wedge \omega^{\delta}$. If $\omega=\sum \varphi_{\bar{J}} d \bar{z}^{J}$ is a tangential $(0,1)$-form on $M$, then $T_{\delta}^{*} \omega$ is a tangential $(0,1)$-form on $M^{\delta}$. Moreover, if $\omega \in \mathcal{C}^{a}(M)$, then

$$
T_{\delta}^{*} \omega=\delta \sum_{\alpha=1}^{n-1} \varphi_{\bar{\alpha}} \circ T_{\delta} d \overline{z^{\alpha}}, \quad \lim _{\delta \rightarrow 0}\left\|\omega^{\delta}\right\|_{\mathcal{C}^{a}\left(D_{1}^{\delta}\right)}=0 .
$$

In other words, we have achieved the smallness of $\omega$ via dilation alone. 
We now consider the integrability condition under a frame change. We are given an $r \times r$ matrix of continuous $(0,1)$-forms $\omega$ on $M$. We assume that $\bar{\partial}_{b} \omega=$ $\omega \wedge \omega \bmod \bar{\partial} r$. Without loss of generality, we may assume that $\omega$ are tangential. The formal integrability condition is that as currents

$$
\bar{\partial}_{M} \omega=\omega \wedge \omega .
$$

Recall that our goal is to find a non-singular matrix $A$ which solves

$$
\bar{\partial}_{M} A+A \omega=0 .
$$

We will consider only the solution $A$ such that both $A$ and $\bar{\partial}_{M} A$ are continuous. For any such $A$, the transformation $\omega \rightarrow \tilde{\omega}=\left(\bar{\partial}_{M} A+A \omega\right) A^{-1}$ preserves the integrability condition (13.2). Indeed, differentiating $\tilde{\omega} A=\bar{\partial}_{M} A+A \omega$ and then using $\omega=A^{-1} \tilde{\omega} A-A^{-1} \bar{\partial}_{M} A, \bar{\partial}_{M} \omega=\omega \wedge \omega$, we verify $\bar{\partial}_{M} \tilde{\omega}=\tilde{\omega} \wedge \tilde{\omega}$ by

$$
\begin{aligned}
\left(\bar{\partial}_{M} \tilde{\omega}\right) A-\tilde{\omega} \wedge \bar{\partial}_{M} A= & \bar{\partial}_{M} A \wedge \omega+A \bar{\partial}_{M} \omega=\bar{\partial}_{M} A \wedge\left(A^{-1} \tilde{\omega} A-A^{-1} \bar{\partial}_{M} A\right) \\
& +\left(\tilde{\omega} A-\bar{\partial}_{M} A\right) \wedge\left(A^{-1} \tilde{\omega} A-A^{-1} \bar{\partial}_{M} A\right) \\
= & \tilde{\omega} \wedge \tilde{\omega} A-\tilde{\omega} \wedge \bar{\partial}_{M} A .
\end{aligned}
$$

Assume that the matrix $\omega$ is of class $\mathcal{C}^{a}$. In what follows, all constants, including $\delta$, will depend on $a$. For simplicity this dependence will not be indicated sometimes. However, constants $C_{0}, \delta_{*}$ and $\epsilon$ do not depend on $a$.

Proof of Theorem 1.1. We need to find a non-singular matrix $A=I+B$, defined near the origin of $M$, such that

$$
\bar{\partial}_{M} B+\omega+B \omega=0 .
$$

It suffices to find a $\delta>0$ and a non-singular matrix $A^{\delta}$ defined near $0 \in M^{\delta}$ such that $\bar{\partial}_{M^{\delta}} \omega^{\delta}+A^{\delta} \omega^{\delta}=0$. Then $A^{\delta} \circ T_{\delta}^{-1}$ is a solution to the original equation.

Take $\rho_{0}=1, \sigma_{j}=2^{-j-1}$ and $\rho_{j+1}=\left(1-\sigma_{j}\right) \rho_{j}$. Then $\rho_{\infty}=\lim _{j \rightarrow \infty} \rho_{j}>$ 0 . We will assume that $0<\delta \leq \delta_{*}$. We want to apply our estimates for $P^{\prime}$ and $Q^{\prime}$. So we choose $\delta_{*} \in(0,1]$ such that the homotopy formula holds on $M_{\rho}^{\delta}$ for $\rho \in(0,1]$ and $\delta \in\left(0, \delta_{*}\right]$. For $\rho_{\infty}<\rho \leq 1$ we have

$$
\left\|P^{\prime} \varphi\right\|_{\mathcal{C}^{a}\left(M_{(1-\sigma) \rho}^{\delta}\right)} \leq C \sigma^{-s}\|\varphi\|_{\mathcal{C}^{a+2}\left(M_{\rho}^{\delta}\right)},
$$

where $P^{\prime}$ is either of operators $P^{\prime}, Q^{\prime}$ in the homotopy formula on $M_{\rho}^{\delta}$. We emphasize that the constant $C_{a}$ is independent of $\delta \in\left(0, \rho_{*}\right)$ and $\rho \in\left(\rho_{\infty}, 1\right]$. We have also absorbed $\left\|r^{\delta}\right\|_{\mathcal{C}^{a+2}\left(D_{\rho_{j}}^{\delta}\right)}$ into $C_{a}$, since $\hat{r}^{\delta}\left(z^{\prime}, x^{n}\right)=\delta^{-2} \hat{r}\left(\delta z^{\prime}, \delta^{2} x^{n}\right)$ and $\hat{r}(0)=\partial \hat{r}(0)=0$ imply that

$$
\left\|\hat{r}^{\delta}\right\|_{\mathcal{C}^{a+2}\left(D_{\rho_{j}}^{\delta}\right)} \leq C\|\hat{r}\|_{\mathcal{C}^{2}\left(D_{\delta \rho_{0}}\right)}+\delta^{a}\|\hat{r}\|_{\mathcal{C}^{a+2}\left(D_{\delta \rho_{0}}\right)}<C_{a}, \quad 0<\delta<\delta_{*} .
$$


Set $M_{j}=M_{\rho_{j}}^{\delta}$ and $\|\cdot\|_{\rho_{j+1}, a}=\|\cdot\|_{\mathcal{C}^{a}\left(M_{j+1}\right)}$. We have $M_{j+1} \subset M_{0}$. Let $\omega_{0}=$ $T_{\delta}^{*} \omega$, restricted on $M_{0}$. On $M_{j}$ we have the homotopy formula $\varphi=\bar{\partial}_{M^{\delta}} P_{j}^{\prime} \varphi+$ $Q_{j}^{\prime} \bar{\partial}_{M^{\delta}} \varphi$, where $P_{j}^{\prime}=P_{M_{j}}^{\prime}$ and $Q_{j}^{\prime}=Q_{M_{j}}^{\prime}$.

Using $\bar{\partial}_{M^{\delta}} \omega_{0}=\omega_{0} \wedge \omega_{0}$, we arrive at the equation

$$
\bar{\partial}_{M^{\delta}}\left(B_{0}+P_{0}^{\prime} \omega_{0}\right)+Q_{0}^{\prime}\left(\omega_{0} \wedge \omega_{0}\right)+B_{0} \omega_{0}=0,
$$

where $P_{0}^{\prime}, Q_{0}^{\prime}$ are applied entrywise to the matrices. We use the approximate solution

$$
B_{0}=-P_{0}^{\prime} \omega_{0} .
$$

Assume that $A_{0}=I+B_{0}$ is invertible. We repeat this procedure and get $B_{j}=$ $-P_{j}^{\prime} \omega_{j}$ and

$$
\omega_{j+1}=\left(\bar{\partial}_{M} A_{j}+A_{j} \omega_{j}\right) A_{j}^{-1}=\left\{Q_{j}^{\prime}\left(\omega_{j} \wedge \omega_{j}\right)-\left(P_{j}^{\prime} \omega_{j}\right) \omega_{j}\right\}\left(I-P_{j}^{\prime} \omega_{j}\right)^{-1} .
$$

Here, we need all $A_{j}=I+B_{j}$ to be non-singular on $M_{j}$. We want to show that $\lim _{j \rightarrow \infty} A_{j} A_{j-1} \cdots A_{0}$ is a solution.

We now estimate $\left\|B_{j}\right\|_{\rho_{j+1}, a}$ and $\left\|\omega_{j+1}\right\|_{\rho_{j+1}, a}$.

For an $r \times r$ matrix $B=\left(b_{i}^{j}\right)$ of functions on $M_{\rho}$ we define $\|B\|_{\rho, a}=$ $\max \left\{\left\|b_{i}^{j}\right\|_{\rho, a}\right\}$. If $B, D$ are two such matrices, we have

$$
\|D B\|_{\rho, 0} \leq r^{2}\|D\|_{\rho, 0}\|B\|_{\rho, 0}, \quad\left\|B^{l}\right\|_{\rho, 0} \leq r^{l}\|B\|_{\rho, 0}^{l} .
$$

Assume that $\rho_{\infty}<\rho \leq \rho_{0}$. We want to show that if $\|B\|_{\rho, 0} \leq \frac{1}{2 r}$, then

$$
\|D\|_{\rho, a} \leq c_{a}\|B\|_{\rho, a}, \quad 0 \leq a<\infty,
$$

where $c_{a}>1$ depends on $a, r, n$. Let $A^{-1}=I+D$. We know that $D=$ $\sum_{l \geq 1}(-1)^{l} B^{l}$ and

$$
\|D\|_{\rho, 0} \leq \frac{r\|B\|_{\rho, 0}}{1-r\|B\|_{\rho, 0}} \leq 2 r\|B\|_{\rho, 0},
$$

which is (13.6) with $a=0$. Since $I$ is constant, then

$$
\begin{aligned}
D\left(z_{2}\right)-D\left(z_{1}\right) & =A\left(z_{2}\right)^{-1}-A\left(z_{1}\right)^{-1}=A\left(z_{1}\right)^{-1}\left(A\left(z_{1}\right)-A\left(z_{2}\right)\right) A\left(z_{2}\right)^{-1} \\
& =A\left(z_{1}\right)^{-1}\left(B\left(z_{1}\right)-B\left(z_{2}\right)\right) A\left(z_{2}\right)^{-1} .
\end{aligned}
$$

Using (13.6) with $a=0$, we get $\|D\|_{\rho, a} \leq C\|B\|_{\rho, a}$ if $0<a \leq 1$. Assume that (13.6) holds when $[a]<k$. Let $[a]=k \geq 1$. Applying the product rule to $(I+B) D=-B$ and multiplying from left by $(I+B)^{-1}=I+D$, we get

$$
\partial D=(I+D)(\partial B) D-(I+D) \partial B .
$$


By Proposition A.4 and the induction assumption, we obtain

$$
\|D\|_{\rho, a} \leq C_{a}\left\{\left(1+\|B\|_{\rho, a-1}\right)\|B\|_{\rho, 1}+\|B\|_{\rho, a}\right\} \leq C_{a}^{\prime}\|B\|_{\rho, a},
$$

which proves (13.6).

Since $B_{j}=-P_{j}^{\prime} \omega_{j}$, by (13.4) we have

$$
\left\|B_{j}\right\|_{\rho_{j+1}, a} \leq c_{a}^{*} \sigma_{j}^{-s_{a}}\left\|\omega_{j}\right\|_{\rho_{j}, a}, \quad c_{a}^{*}>1 .
$$

We want to achieve $\left\|B_{j}\right\|_{\rho_{j+1}, 0} \leq \frac{1}{2 r}$. So it suffices to obtain

$$
\left\|\omega_{j}\right\|_{\rho_{j}, a} \leq \frac{\sigma_{j}^{s_{a}}}{2 r c_{a}^{*} c_{a}}=b_{j}, \quad j=0,1,2, \ldots
$$

By (13.6)-(13.8), we have $\left\|(I+B)^{-1}\right\|_{\rho_{j}, a} \leq 1+\|D\|_{\rho_{j}, a} \leq 2$. Using (13.4)-(13.5) and the estimates on matrix products, we get

$$
\begin{aligned}
\left\|\omega_{j+1}\right\|_{\rho_{j+1}, a} \leq & 2 \cdot r^{2}\left\{\left\|Q_{j}^{\prime}\left(\omega_{j} \wedge \omega_{j}\right)\right\|_{\rho_{j+1}, a}\right. \\
& \left.+r^{2}\left\|B_{j}\right\|_{\rho_{j+1}, a}\left\|\omega_{j}\right\|_{\rho_{j+1}, a}\right\} \leq C_{a}^{*} \sigma_{j}{ }^{-s_{a}}\left\|\omega_{j}\right\|_{\rho_{j}, a}^{2} .
\end{aligned}
$$

Assume that $\left\|\omega_{0}\right\|_{\rho_{0}, a} \neq 0$. Otherwise the theorem holds trivially. Define

$$
\hat{b}_{j+1}=C_{a}^{*} \sigma_{j}^{-s_{a}} \hat{b}_{j}^{2}, \quad \hat{b}_{0}=\left\|\omega_{0}\right\|_{\rho_{0}, a} .
$$

By (13.1), we choose a dilation $T_{\delta}$ with $\delta \in\left(0, \delta_{*}\right]$ such that $\omega_{0}=T_{\delta} \omega$ satisfies

$$
\hat{b}_{0}=\left\|\omega_{0}\right\|_{\rho_{0}, a} \leq b_{0} .
$$

Then $r_{j}=\hat{b}_{j+1} / \hat{b}_{j}$ satisfies

$$
\begin{gathered}
r_{0}=C_{a}^{*} \sigma_{0}^{-s_{a}} \hat{b}_{0}, \quad r_{j}=2^{s_{a}}\left(r_{j-1}\right)^{2}, \\
r_{1} / r_{0}=2^{s_{a}} r_{0}, \quad r_{j+1} / r_{j}=\left(r_{j} / r_{j-1}\right)^{2} .
\end{gathered}
$$

This shows that $r_{j+1} / r_{j}$, and hence $r_{j}, \hat{b}_{j}$, converge rapidly, if $\hat{b}_{0}$ is sufficiently small. Specifically,

$$
r_{j+1} / r_{j}=\left(r_{1} / r_{0}\right)^{2^{j}}, \quad r_{j}=r_{0}\left(r_{1} / r_{0}\right)^{2^{j}-1}, \quad \hat{b}_{j}=\hat{b}_{0} r_{0}^{j}\left(r_{1} / r_{0}\right)^{2^{j}-j-1}
$$

Recall that $\sigma_{j}=2^{-j-1}$. Clearly, $\left\|\omega_{j}\right\|_{\rho_{j}, a} \leq \hat{b}_{j} \leq \hat{b}_{0} r_{0}^{j} \leq \frac{2^{-s_{a}}}{2 r c_{a}^{*} c_{a}}\left(2^{-s_{a}}\right)^{j}=b_{j}$, provided

$$
\hat{b}_{0} \leq \frac{2^{-s_{a}}}{2 r c_{a}^{*} c_{a}}, \quad r_{0} \leq 2^{-s_{a}}, \quad r_{1} / r_{0}=2^{s_{a}} r_{0} \leq \frac{1}{2}
$$


Therefore, we have shown that if $\omega \in \mathcal{C}^{a}$ and $M \in \mathcal{C}^{2+a}$ then $\left(I+B_{j}\right) \cdots\left(I+B_{0}\right)$ converges in $\mathcal{C}^{a}$-norm on $\cap D_{\rho_{j}}^{\delta}$ to an invertible matrix $A_{\infty}$. When $a=k$ is an integer and $M \in \mathcal{C}^{k+5 / 2}$, or when $M \in \mathcal{C}^{2}$ for $k=0$, we have

$$
\left\|B_{j}\right\|_{\rho_{j+1}, k+1 / 2} \leq c_{k} \sigma_{j}^{-s_{k}}\left\|\omega_{j}\right\|_{\rho_{j}, k} .
$$

Since $\left\|\omega_{j}\right\|_{\rho_{j}, k}$ tends to zero rapidly, it is obvious that $B_{j}$ converges to 0 rapidly in $\mathcal{C}^{k+1 / 2}$ on $\cap D_{\rho_{j}}^{j}$. This shows that $A^{\infty}$ is of class $\mathcal{C}^{k+1 / 2}$.

To complete the proof, we check that $\bar{\partial}_{M^{\delta}} A^{\infty}+A^{\infty} \omega_{0}=0$. Recall that for $A_{j}=I+B_{j}$, we have $\bar{\partial}_{M^{\delta}} A_{j}+A_{j} \omega_{j}=w_{j+1} A_{j}$. Thus

$$
\begin{aligned}
\omega_{j+1} A_{j} A_{j-1} & =\left(\bar{\partial}_{M^{\delta}} A_{j}\right) A_{j-1}+A_{j} \omega_{j} A_{j-1} \\
& =\left(\bar{\partial}_{M^{\delta}} A_{j}\right) A_{j-1}+A_{j}\left(\bar{\partial}_{M^{\delta}} A_{j-1}+A_{j-1} \omega_{j-1}\right) \\
& =\bar{\partial}_{M^{\delta}}\left(A_{j} A_{j-1}\right)+A_{j} A_{j-1} \omega_{j-1} .
\end{aligned}
$$

Inductively, we get $\bar{\partial}_{M^{\delta}}\left(A_{j} \cdots A_{0}\right)+A_{j} \cdots A_{0} \omega_{0}=\omega_{j+1} A_{j} \cdots A_{0}$. Taking the limits, we get $\bar{\partial}_{M^{\delta}} A^{\infty}+A^{\infty} \omega_{0}=0$ on $M_{\rho_{\infty}}^{\delta}$. When $k=0$, the derivatives in the sense of currents are continuous and the above computation is valid as currents.

In the above argument, we obtain the rapid convergence of $B_{j}, \omega_{j}$ in $\mathcal{C}^{a}$ norm in one step when $a$ is finite. One can also establish a rapid convergence of $\omega_{j}, B_{j}$ first in $\mathcal{C}^{0}$-norm and then in higher order derivatives. See [23], [5] for details.

\section{Appendix}

\section{A. Hölder inequalities}

The main purpose of this appendix is to present some Hölder inequalities on domains in $\mathbb{R}^{m}$. We do not claim any originality in deriving these inequalities. In fact, we will just modify formulation and proofs of Hörmander [9]. The inequalities in [9] are for a fixed convex domain. In our applications, we need to allow the domain $D_{\rho}$ to vary. Therefore, we will derive them in full details and omit simple repetitions only.

We say that a domain $D$ in $\mathbb{R}^{m}$ has the cone property if the following hold: (i) Given two points $p_{0}, p_{1}$ in $D$ there exists a piecewise $\mathcal{C}^{1}$ curve $\gamma(t)$ in $D$ such that $\gamma(0)=p_{0}$ and $\gamma(1)=p_{1},\left|\gamma^{\prime}(t)\right| \leq C_{*}\left\|p_{1}-p_{0}\right\|$ for all $t$ except finitely many values. The diameter of $D$ is less than $C_{*}$. (ii) For each point $x \in \bar{D}, D$ contains a cone $V$ with vertex $x$, opening $\theta>C_{*}^{-1}$ and height $h>C_{*}^{-1}$.

We will denote $C_{*}(D)$ a constant $C_{*}>1$ satisfying (i) and (ii). 
In this appendix, by a cone $V=V(\theta, h, v)$ with vertex at the origin, opening $\theta>0$ and height $h>0$, and centered at positive $v$ axis where $v$ is a unit vector, we mean

$$
V=\left\{t \in \mathbb{R}^{m}: v \cdot t>\theta^{-1}\|t-(v \cdot t) v\|, v \cdot t<h\right\},
$$

where $\|t\|=\left(\left|t^{1}\right|^{2}+\cdots+\left|t^{m}\right|^{2}\right)^{\frac{1}{2}}$. Note that $x+V$ is a cone with vertex at $x$. Note that each cone satisfying (ii) contains a ball of radius at least $C_{*}(D) / C$. Let $D_{1}, D_{2}$ be domains of the cone property, and let $V_{1}, V_{2}$ be cones in (ii) for $D_{1}, D_{2}$ respectively. Assume that the vertex of $V_{i}$ is $p_{i}$. Then $D_{1} \times D_{2}$ contains the convex hull of $\left(p_{1}, p_{2}\right)$ and a ball of radius depending only on $C_{*}\left(D_{1}\right), C_{*}\left(D_{2}\right)$. Therefore, $D_{1} \times D_{2}$ still has the cone property.

For the rest of the appendix, until Proposition A.5, we assume that the domain $D$ has the cone property unless stated otherwise. The constants in all Hölder inequalities will depend on $m$ and $C_{*}(D)$. For simplicity this dependence will not be expressed sometimes.

Let $k \geq 0$ be an integer. For a complex-valued function $u$ on $D \subset \mathbb{R}^{m}$, define

$$
\begin{gathered}
\left\|\partial^{k} u\right\|_{D, 0}=\sup _{x \in D,|I|=k}\left|\partial^{I} u(x)\right|, \quad\|u\|_{D, k}=\max _{0 \leq j \leq k}\left\|\partial^{j} u\right\|_{D, 0}, \\
|u|_{D, \alpha}=\sup _{x, y \in D} \frac{|u(x)-u(y)|}{|x-y|^{\alpha}}, \quad 0<\alpha \leq 1, \\
\|u\|_{D, k+\alpha}=\max \left\{\|u\|_{D, k},\left|\partial^{I} u\right|_{D, \alpha}:|I|=k\right\}, \quad 0<\alpha<1 .
\end{gathered}
$$

If $A=\left(a_{i}^{j}\right)$ is a matrix of functions on $D$, we define $\|A\|_{D, k+\alpha}=\max \left\{\left\|a_{i}^{j}\right\|_{D, k+\alpha}\right\}$.

We also define

$$
|u|_{D, k+\alpha}=\max _{|I|=k}\left|\partial^{I} u\right|_{D, \alpha}, \quad 0<\alpha \leq 1 .
$$

By (i) of the cone property and the fundamental theorem of calculus

$$
C_{0}^{-1}|u|_{D, k} \leq\left\|\partial^{k} u\right\|_{D, 0} \leq|u|_{D, k}, \quad k=1,2, \ldots,
$$

provided that $u \in \mathcal{C}^{k}(D)$. It will be convenient to use both $|u|_{D, k}$ and $\left\|\partial^{k} u\right\|_{D, 0}$.

Lemma A.1. Let $D \subset \mathbb{R}^{m}$ satisfy $(i)$ of the cone property. Let $f$ be a $\mathcal{C}^{1}$ map from $D$ into $\mathbb{R}^{m}$. There exists a constant $C_{0}>1$ such that if $\left|f^{\prime}-I\right|<C_{0}^{-1}$ on $D$, then $f$ is a $\mathcal{C}^{1}$ diffeomorphism from $D$ onto $D^{\prime}$. Moreover, $\left\|f^{-1}-I\right\|_{D^{\prime}, 1} \leq C\|f-I\|_{D, 1}$.

Proof. Take two points $p_{0}, p_{1}$ in $D$. By assumption there exists a piecewise $C^{1}$ curve $\gamma$ in $D$ such that $\gamma(0)=p_{0}, \gamma(1)=p_{1}$, and $\left|\gamma^{\prime}(t)\right| \leq C_{*}\left\|p_{1}-p_{0}\right\|$. Write $f=I+\tilde{f}$. We have

$$
f\left(p_{1}\right)-f\left(p_{0}\right)=p_{1}-p_{0}-\int_{0}^{1} \nabla \tilde{f}(\gamma(t)) \cdot \gamma^{\prime}(t) d t .
$$


Since $\left\|\tilde{f}^{\prime}\right\|_{D, 0}<C_{0}^{-1}$, then for $C_{0}$ sufficiently large we get

$$
\frac{1}{2}\left\|p_{1}-p_{0}\right\| \leq\left\|f\left(p_{1}\right)-f\left(p_{0}\right)\right\| \leq 2\left\|p_{1}-p_{0}\right\| .
$$

Now it is obvious that $f$ is a $\mathcal{C}^{1}$ diffeomorphism from $D$ onto $D^{\prime}$ and that $D^{\prime}$ satisfies (i) of the cone property. Let $\tilde{g}=f^{-1}-I$. Then $\tilde{g} \circ f=-\tilde{f}$. In particular, $\|\tilde{g}\|_{D^{\prime}, 0} \leq\|\tilde{f}\|_{D, 0}$, and by (A.3), $|\tilde{g}|_{D^{\prime}, 1} \leq C|\tilde{f}|_{D, 1}$.

We are ready to derive Hölder inequalities. We start with two lemmas in [9] for our domains.

Lemma A.2. Let $0<a<b$. Let $D$ satisfy the cone property. If $|u|_{D, a} \leq 1$ and $|u|_{D, b} \leq 1$ then $|u|_{D, c} \leq C$ for $a<c<b$, where $C$ depends only on $a, b$ and $C_{*}(D)$.

Proof. For simplicity, denote $|u|_{D, a}$ by $|u|_{a}$. Since the diameter of $D$ is bounded by some constant $C$, it is obvious that $|u|_{c} \leq C^{\prime}|u|_{c^{\prime}}$ if $k<c<c^{\prime} \leq k+1$. Therefore, it suffices to prove the inequality when $c$ is an integer.

Let $V \subset D$ be a cone as stated in the cone property. We may assume that $0 \in V$.

If $a$ is an integer, we get $\left|\partial^{a} u\right| \leq 1$ on $V$. If $a$ is not an integer, let $P$ be its Taylor polynomial of degree $[a]$ at 0 . Set $v=u-P$. Since $\partial^{I} P$ are constants for all $|I| \geq[a]$, then $|v|_{c^{\prime}}=|u|_{c^{\prime}}$ for all $c^{\prime}>[a]$. Therefore, we may assume that $\partial^{I} u(0)=0$ for all $|I|=[a]$. Now $|u|_{a} \leq 1$ implies that $\left|\partial^{I} u\right|<C_{0}$ for all $|I|=[a]$.

We want to use the mean-value-theorem repeatedly. Let us first look at the one-variable case. When $f$ is $\mathcal{C}^{k}$ on $[0,1]$ and $\|f\|_{0} \equiv\|f\|_{[0,1], 0}<C_{0}$, there is a point $t$ in $[0,1]$ such that $\left|f^{\prime}(t)\right| \leq 2 C_{0}$. One can divide [0,1] into a sufficient number of equal parts and find a point $t_{j} \in[0,1]$ such that $\left|f^{(j)}\left(t_{j}\right)\right|<C^{\prime}$ for $j=$ $1, \ldots, k$. If $|f|_{a} \leq 1$ for some $a \in(k, k+1]$, we obtain further that $\left\|f^{(k)}\right\|_{0}<C_{k}$. Consequently, $\left\|f^{(j)}\right\|_{0}<C_{j}$ hold for $j=k, k-1, \ldots, 0$.

Return to our case. Fix a polydisc $\Delta^{m}$ in $V$ with side larger than $C^{-1}$. Fix $I$ with $|I|=[a]$. Using $\left|\partial^{I} u\right|<C_{0}$ for $|I|=[a]$ and $|u|_{b} \leq 1$, by the onevariable argument we obtain $\left|\partial^{j} \partial^{I} u\right|<C$ on $\Delta^{m}$ for $j=[b]-[a], \ldots, c-[a]$. So $\left|\partial^{j} u\right|<C$ on $\Delta^{m}$ for $j=c, \ldots$, [b]. If $[b]<b,|u|_{b} \leq 1$ implies that $\left|\partial^{[b]} u\right|<C^{\prime}$ on $D$. If $b=[b]$ the assumption and (A.2) implies $\left|\partial^{b} u\right| \leq 1$. Using a path connecting a point in $D$ to $\Delta^{m}$ and $\left|\partial^{j} u\right|<C$ on $\Delta^{m}$ for $j=[b], \ldots, c$, we get $\left|\partial^{j} u\right| \leq C^{\prime \prime}$ on $D$ for $j=[b]-1, \ldots, c$. Using (A.2) again, we get $|u|_{c} \leq C$.

Example. Let $f(x)=x+x^{3}$ and $D=[0, \epsilon]$. Then $f^{\prime}(0)=f^{\prime}(x)-\int_{0}^{x} f^{\prime \prime}(t) d t$ and

$$
\epsilon f^{\prime}(0)=\int_{0}^{\epsilon} f^{\prime}(x) d x-\int_{0}^{\epsilon} \int_{0}^{x} f^{\prime \prime}(t) d t d x=f(\epsilon)-f(0)-\int_{0}^{\epsilon} \int_{0}^{x} f^{\prime \prime}(t) d t d x .
$$


This shows that $1=\left|f^{\prime}(0)\right| \leq C_{1}\|f\|_{0}+C_{2}\left\|f^{\prime \prime}\right\|_{0} \leq 2 \epsilon C_{1}+6 \epsilon C_{2}$. However, $\left|C_{1}\right|+\left|C_{2}\right|$ tends to $\infty$ as $\epsilon \rightarrow 0$. This example demonstrates that in some inequalities derived in this appendix, the constants indeed depend on $C_{*}(D)$. For special domains used in this paper, we will find these constants by dilation; see Proposition A.5.

Lemma A.3. Let $D \subset \mathbb{R}^{m}$ satisfy the cone property. Set $\|\cdot\|_{D, a}=\|\cdot\|_{a}$. If $0<a<b, c=\lambda a+(1-\lambda) b$ and $0<\lambda<1$, then $|u|_{c} \leq C|u|_{a}^{\lambda}\left(|u|_{a}+|u|_{b}\right)^{1-\lambda}$, where $C$ depends only on $a, b$.

Proof. The case $|u|_{a} \geq|u|_{c}$ is obvious and we may assume that $|u|_{a} \leq|u|_{c}$. If $|u|_{a}=0$, then $u$ is a polynomial of degree $<a$. Then $|u|_{c}=0$ too for $c>a$, and the inequality holds. We may assume that $|u|_{a} \neq 0$. Without loss of generality, we may assume that $|u|_{a}=1<|u|_{c}$. If $|u|_{b} \leq 1$, Lemma A.2 implies that $|u|_{c} \leq C$ and the inequality holds. Therefore, it suffices to verify

$$
|u|_{c} \leq C|u|_{b}^{1-\lambda}, \quad \text { if }|u|_{a}=1<|u|_{b} .
$$

We first assume the inequality for integer $c$ and verify it for non-integer $c$. Set $[c]=k$. We have $\lambda=\frac{b-c}{b-a}$ and $1-\lambda=\frac{c-a}{b-a}$. Depending on whether $a, b$ are in $[k, k+1]$, we have the following cases.

Case i) $k \leq a<c<b \leq k+1$. Since $c=\lambda a+(1-\lambda) b$ then $c-k=$ $\lambda(a-k)+(1-\lambda)(b-k)$. Consider first the case $a=k$. Then $c-k=(1-\lambda)(b-k)$. Let $v=\partial^{I} u$ with $|I|=k$. Hence

$$
\begin{aligned}
\frac{|v(y)-v(x)|}{|y-x|^{c-k}} & =|v(y)-v(x)|^{\lambda}\left|\frac{v(y)-v(x)}{|y-x|^{b-k}}\right|^{1-\lambda} \leq 2^{\lambda}\|v\|_{0}^{\lambda}|v|_{b-k}^{1-\lambda} \\
& \leq C^{\prime}|u|_{k}^{\lambda}\left|\partial^{I} u\right|_{b-k}^{1-\lambda} \leq C^{\prime}|u|_{k}^{\lambda}|u|_{b}^{1-\lambda},
\end{aligned}
$$

where the second last inequality is obtained by (A.2) using $k=a>0$. Therefore, $|u|_{c} \leq C|u|_{a}^{\lambda}|u|_{b}^{1-\lambda}$. Assume now that $a-k>0$. Then $a-k, b-k, c-k$ are in $(0,1]$. Computing the Hölder ratio gives us $|v|_{c-k} \leq|v|_{a-k}^{\lambda}|v|_{b-k}^{1-\lambda}$, i.e. $|u|_{c} \leq|u|_{a}^{\lambda}|u|_{b}^{1-\lambda}$. We emphasize that we have proved $|u|_{c} \leq C|u|_{a}^{\lambda}|u|_{b}^{1-\lambda}$ (and hence (A.4) for case i)) without using any condition on $|u|_{a},|u|_{b},|u|_{c}$ other than that on $a, b, c, k$.

Case ii) $a<k<c<b \leq k+1$. We get $|u|_{c} \leq C|u|_{k}^{(b-c) /(b-k)}|u|_{b}^{(c-k) /(b-k)}$, by case i). By the assumption for the integer case (applied to triple $a<k<b$ ) we obtain

$$
|u|_{k} \leq C|u|_{b}^{(k-a) /(b-a)} .
$$

Eliminating $|u|_{k}$ from two inequalities gives us (A.4); indeed

$$
\frac{k-a}{b-a} \cdot \frac{b-c}{b-k}+\frac{c-k}{b-k}=\frac{c-a}{b-a} .
$$


Case iii) $k \leq a<c<k+1<b$. We have $|u|_{c} \leq C|u|_{k+1}^{(c-a) /(k+1-a)}$, by case i). The assumption on the integer case (applied to triple $a<k+1<b$ ) gives us

$$
|u|_{k+1} \leq C|u|_{b}^{(k+1-a) /(b-a)} .
$$

Eliminating $|u|_{k+1}$ gives us (A.4).

Case iv) $a<k<c<k+1<b$. Then (A.5) and (A.6) are still valid. By i), we have $|u|_{c} \leq C|u|_{k}^{k+1-c}|u|_{k+1}^{c-k}$. Using (A.5)-(A.6) and eliminating $|u|_{k},|u|_{k+1}$ gives us (A.4). in [9].

Finally, we prove (A.4) when $c$ is a positive integer by repeating an argument

Fix $x_{0} \in D$ and let $V$ be a cone in $D$ with vertex $x_{0}$, height and opening $1 / C_{*}$. Since $V$ is convex, for $x \in V$ and $0<\epsilon<1$ we can define

$$
u_{x}^{\epsilon}(y)=u((1-\epsilon) x+\epsilon y), \quad y \in V .
$$

Then $\left|u_{x}^{\epsilon}\right|_{V, b} \leq \epsilon^{b}|u|_{b}$ and $\left|u_{x}^{\epsilon}\right|_{V, a} \leq \epsilon^{a}$. Since $|u|_{b}>1$, there is an $\epsilon \in(0,1)$ so that $\epsilon^{a}=\mu=\epsilon^{b}|u|_{b}$. Now, apply Lemma A.2 to the domain $V$ and the function $\mu^{-1} u_{x}^{\epsilon}$. For any multiindex $I$ with $|I|=c$, we have

$$
\epsilon^{c}\left|\partial^{I} u(x)\right|=\left|\partial^{I} u_{x}^{\epsilon}(x)\right| \leq C_{0} \mu=C_{0}\left(\epsilon^{a}\right)^{\lambda}\left(\epsilon^{b}|u|_{b}\right)^{1-\lambda} .
$$

Canceling $\epsilon$ 's shows $\left|\partial^{I} u(x)\right| \leq C_{0}|u|_{b}^{1-\lambda}$ for $x \in \bar{V}$. Since $C_{0}$ does not depend on $x_{0} \in \bar{V}$, we get $\left\|\partial^{c} u\right\|_{0} \leq C_{0}|u|_{b}^{1-\lambda}$ on $D$; by (A.2), $|u|_{c} \leq C\left\|\partial^{c} u\right\|_{0}$ and (A.4) follows.

Proposition A.4. Let $D \subset \mathbb{R}^{m}$ have the cone property and denote $\|\cdot\|_{D, a}$ by $\|\cdot\|_{a}$. Let $a, b, a_{j}, b_{j}$ be nonnegative real numbers.

(i) $\|u\|_{\lambda a+(1-\lambda) b} \leq C_{a, b}\|u\|_{a}^{\lambda}\|u\|_{b}^{1-\lambda}$ for $0<\lambda<1$.

(ii) $\|u v\|_{a} \leq C_{a}\left(\|u\|_{0}\|v\|_{a}+\|u\|_{a}\|v\|_{0}\right)$.

(iii) Suppose that $D_{j} \subset \mathbb{R}^{n_{j}}$ has the cone property for $j=1, \ldots, k$. Let $a_{j}, c_{j}$ be non-negative real numbers, and let $\left(b_{1}, \ldots, b_{k}\right)$ be in the convex hull of $\left(a_{1}, \ldots, a_{k}\right)$ and $\left(c_{1}, \ldots, c_{k}\right)$. Then

$$
\prod_{j=1}^{k}\left\|u_{j}\right\|_{D_{j}, b_{j}} \leq C_{k+|a|+|b|+|c|}\left(\prod_{j=1}^{k}\|u\|_{D_{j}, a_{j}}+\prod_{j=1}^{k}\|u\|_{D_{j}, c_{j}}\right) .
$$

(iv) Let $f$ be a map from $D$ into $D^{\prime} \subset \mathbb{R}^{n}$. Assume that $D^{\prime}$ has the cone property. Then

$$
\begin{aligned}
& \|u \circ f\|_{a} \leq C_{a}\left(\left\|u^{\prime}\right\|_{D^{\prime}, a-1}\left\|f^{\prime}\right\|_{0}^{a}+\left\|u^{\prime}\right\|_{D^{\prime}, 0}\left\|f^{\prime}\right\|_{a-1}\right)+\|u\|_{D^{\prime}, 0}, \quad a \geq 1, \\
& \|u \circ f\|_{a} \leq C \min \left(\left\|u^{\prime}\right\|_{D^{\prime}, 0}|f|_{a},\|u\|_{D^{\prime}, a}\left\|f^{\prime}\right\|_{0}^{a}\right)+\|u\|_{D^{\prime}, 0}, \quad 0 \leq a \leq 1 .
\end{aligned}
$$


(v) Let $f=I+\tilde{f}$ be a $C^{a}$ map from $D$ into $\mathbb{R}^{m}$. There exists $C_{0}>1$ such that if $\left|\tilde{f}^{\prime}\right|<C_{0}^{-1}$ then

$$
\left\|f^{-1}-I\right\|_{f(D), a} \leq C_{a}\|\tilde{f}\|_{a}, \quad a \geq 0 .
$$

(vi) Let $f=I+\tilde{f}$ be a $C^{1}$ map from $D$ into $D^{\prime} \subset \mathbb{R}^{m}$ with $\left|f^{\prime}\right|<C_{0}$. Assume that $D \cup D^{\prime}$ is contained in a convex domain $D^{\prime \prime}$ of the cone property. Then

$$
\|u \circ f-u\|_{a} \leq C_{a}\left(\left\|u^{\prime}\right\|_{D^{\prime \prime}, a}\|\tilde{f}\|_{0}+\left\|u^{\prime}\right\|_{D^{\prime \prime}, 0}\|\tilde{f}\|_{a}\right), \quad a \geq 0 .
$$

Proof. (i)-(iii) are proved in [9]. The proof for (iii) is for $D_{j}$ being the same domain. In fact, by (i), one has $\log \left\|u_{j}\right\|_{D_{j}, b_{j}} \leq \lambda \log \left\|u_{j}\right\|_{D_{j}, a_{j}}+(1-\lambda) \log \left\|u_{j}\right\|_{D_{j}, c_{j}}+$ $\log C$ for all $j$. Sum over $j=1, \ldots, k$ and take exponential on both sides. The convexity of $e^{x}$ yields (iii). For $0 \leq a \leq 1$, two inequalities in (iv) are verified directly. Assume that (iv) holds when $a$ is replaced by $a-1$. Assume that $a>1$. Then $(u \circ f)^{\prime}=u^{\prime}(f) f^{\prime}$. For both cases of $0<a-1 \leq 1$ and $a-1>1$ we obtain

$$
\begin{aligned}
\left\|(u \circ f)^{\prime}\right\|_{a-1} & \leq C\left(\left\|u^{\prime}(f)\right\|_{a-1}\left\|f^{\prime}\right\|_{0}+\left\|u^{\prime}(f)\right\|_{0}\left\|f^{\prime}\right\|_{a-1}\right) \\
& \leq C^{\prime}\left(\left\|u^{\prime}\right\|_{D^{\prime}, a-1}\left\|f^{\prime}\right\|_{0}^{a}+\left\|u^{\prime}\right\|_{0}\left\|f^{\prime}\right\|_{a-1}\right),
\end{aligned}
$$

where $\left\|u^{\prime}\right\|_{a-1} \equiv\left\|u^{\prime}\right\|_{D^{\prime}, a-1}$. Note that for the second inequality when $a>2$, we have used

$$
\begin{aligned}
\left\|u^{\prime}\right\|_{1}\left\|f^{\prime}\right\|_{a-2}\left\|f^{\prime}\right\|_{0} & \leq C\left\|u^{\prime}\right\|_{0}^{\frac{a-2}{a-1}}\left\|u^{\prime}\right\|_{a-1}^{\frac{1}{a-1}}\left\|f^{\prime}\right\|_{0}^{\frac{1}{a-1}}\left\|f^{\prime}\right\|_{a-1}^{\frac{a-2}{a-1}}\left\|f^{\prime}\right\|_{0} \\
& \leq C^{\prime}\left(\left\|u^{\prime}\right\|_{a-1}\left\|f^{\prime}\right\|_{0}^{a}+\left\|u^{\prime}\right\|_{0}\left\|f^{\prime}\right\|_{a-1}\right) .
\end{aligned}
$$

This gives us (iv) as $\|u \circ f\|_{a} \leq\|u\|_{0}+\left\|(u \circ f)^{\prime}\right\|_{a-1}$.

(v). It follows immediately from Lemma A.1 when $0 \leq a \leq 1$. We now prove it for $a>1$ by using a variant of counting scheme in Section 2. Define

$$
\widehat{\partial}^{1+k} v=\sum_{j_{1}+\cdots+j_{l} \leq k} p\left(\tilde{f}^{\prime}\right) \partial^{J_{1}} v \cdots \partial^{J_{l}} v, \quad j_{i}=\left|J_{i}\right|-1 \geq 0,
$$

where $p\left(\tilde{f}^{\prime}\right)$ is a polynomial in $\left(I+\tilde{f}^{\prime}\right)^{-1}$ and $\tilde{f}^{\prime}$ and it might be different when it reoccurs. Let $g=f^{-1}$ and $\tilde{g}=f^{-1}-I$. Let 1 denote the identity matrix. We have $\tilde{g}^{\prime}=-\left(\left(\mathbf{1}+\tilde{f}^{\prime}\right)^{-1} \tilde{f}^{\prime}\right) \circ g$, i.e. $\partial^{I} \tilde{g}=\left(\widehat{\partial}^{1} \tilde{f}\right) \circ g$ for $|I|=1$. Inductively,

$$
\partial^{J} \tilde{g}=\left(\widehat{\partial}^{|J|} \tilde{f}\right) \circ g, \quad|J| \geq 1 .
$$

By Lemma A.1, we have $\frac{1}{2}\left|y_{1}-y_{0}\right| \leq\left|g\left(y_{1}\right)-g\left(y_{0}\right)\right| \leq 2\left|y_{1}-y_{0}\right|$. Let $k=[a]-1$ and $\alpha=a-k-1$. Thus $\|g\|_{D^{\prime}, 1+k+\alpha} \leq C\|f\|_{0}+C \sum_{j \leq k}\left\|\widehat{\partial}^{1+j} \tilde{f}\right\|_{\alpha}$. Now

$$
\begin{aligned}
\left\|\widehat{\partial}^{1+k} \tilde{f}\right\|_{\alpha} \leq & \sum_{j_{1}+\ldots+j_{l} \leq k} C^{\prime}\left\{\|\tilde{f}\|_{1+\alpha}\|\tilde{f}\|_{1+j_{1}} \cdots\|\tilde{f}\|_{1+j_{l}}\right. \\
& \left.+\sum_{1 \leq i \leq l}\|\tilde{f}\|_{1+j_{1}} \cdots\|\tilde{f}\|_{1+j_{i}+\alpha} \cdots\|\tilde{f}\|_{1+j_{l}}\right\} .
\end{aligned}
$$

By (iii) we obtain $\|\tilde{g}\|_{D^{\prime}, 1+k+\alpha} \leq \sum C\left(\|\tilde{f}\|_{1}^{l}+\|\tilde{f}\|_{1}^{l-1}\right)\|\tilde{f}\|_{1+k+\alpha} \leq C^{\prime}\|\tilde{f}\|_{a}$. 
(vi). By convexity of $D^{\prime \prime}$, we have $u(x+\tilde{f}(x))-u(x)=\tilde{f}(x) \cdot \int_{0}^{1} u^{\prime}(x+t \tilde{f}(x)) d t$. Consider case $0 \leq a<1$. We have

$$
\begin{aligned}
\left|\int_{0}^{1}\left\{u^{\prime}(y+t \tilde{f}(y))-u^{\prime}(x+t \tilde{f}(x))\right\} d t\right| & \leq \max _{t}\left\|u^{\prime}\right\|_{D^{\prime \prime}, a}|y-x+t(\tilde{f}(y)-\tilde{f}(x))|^{a} \\
& \leq C\left\|u^{\prime}\right\|_{D^{\prime \prime}, a}\left(1+\|\tilde{f}\|_{D, 1}\right)|y-x|^{\alpha} .
\end{aligned}
$$

By $\|\tilde{f}\|_{1} \leq C$, one sees easily that

$$
\|u(I+\tilde{f})-u\|_{a} \leq C\left(\left\|u^{\prime}\right\|_{D^{\prime \prime}, a}\|\tilde{f}\|_{0}+\left\|u^{\prime}\right\|_{D^{\prime \prime}, 0}\|\tilde{f}\|_{a}\right),
$$

which is (vi) for $0 \leq a<1$. Assume that the above inequality holds when $a$ is replaced by $a-1$. Assume that $a \geq 1$. We need to estimate the $\|\cdot\|_{a-1}$ norm of

$$
\partial_{x^{i}}(u \circ(I+\tilde{f})-u)=\left(\partial_{y^{i}} u\right) \circ(I+\tilde{f})-\partial_{x^{i}} u+\sum_{1 \leq j \leq m}\left(\partial_{y^{j}} u\right) \circ(I+\tilde{f}) \cdot \partial_{x^{i}} \tilde{f}^{j} .
$$

By the induction assumption, we have

$$
\left\|\left(\partial_{y^{i}} u\right) \circ(I+\tilde{f})-\partial_{x^{i}} u\right\|_{a-1} \leq C\left(\left\|\partial^{2} u\right\|_{D^{\prime \prime}, a-1}\|\tilde{f}\|_{0}+\left\|\partial^{2} u\right\|_{D^{\prime \prime}, 0}\|\tilde{f}\|_{a-1}\right) .
$$

We need to put $\left\|\partial^{2} u\right\|_{D^{\prime \prime}, l}\|\tilde{f}\|_{a-1-l} \leq\left\|u^{\prime}\right\|_{D^{\prime \prime}, l+1}\|\tilde{f}\|_{a-1-l}$ into the desired form. By (iii), we get

$$
\left\|u^{\prime}\right\|_{D^{\prime \prime}, l+1}\|\tilde{f}\|_{a-1-l} \leq C\left(\|\tilde{f}\|_{0}\left\|u^{\prime}\right\|_{D^{\prime \prime}, a}+\left\|u^{\prime}\right\|_{D^{\prime \prime}, 0}\|\tilde{f}\|_{a}\right) .
$$

We now treat the term in the sum of (A.9). Set $\left\|u^{\prime}\right\|_{b} \equiv\left\|u^{\prime}\right\|_{D^{\prime \prime}, b}$. By (iv) we have $\left\|\left(\partial_{y^{j}} u\right)(I+\tilde{f})\right\|_{a-1} \leq C\left(\left\|u^{\prime}\right\|_{a-1}+\left\|u^{\prime}\right\|_{1}\|\tilde{f}\|_{a-1}\right)+\left\|u^{\prime}\right\|_{0}$. Thus

$$
\begin{aligned}
\left\|\left(\partial_{y^{j}} u\right)(I+\tilde{f}) \cdot \partial_{x^{i}} \tilde{f}^{j}\right\|_{a-1} & \leq C\left(\left(\left\|u^{\prime}\right\|_{a-1}+\left\|u^{\prime}\right\|_{1}\|\tilde{f}\|_{a-1}\right)\|\tilde{f}\|_{1}+\left\|u^{\prime}\right\|_{0}\|\tilde{f}\|_{a}\right) \\
& \leq C^{\prime}\left(\left\|u^{\prime}\right\|_{a-1}\|\tilde{f}\|_{1}+\left\|u^{\prime}\right\|_{1}\|\tilde{f}\|_{a-1}+\left\|u^{\prime}\right\|_{0}\|\tilde{f}\|_{a}\right) .
\end{aligned}
$$

We can put the first two terms in the desired form by (A.10).

Two inequalities in the next proposition are used in estimating $P$ and $Q$.

Proposition A.5. Let $D$ be a convex domain in $\mathbb{R}^{m}$ satisfying

$$
B_{\rho / c_{0}} \subset D \subset B_{c_{0} \rho}, \quad 0<\rho \leq 3 .
$$

Let $a_{*}=0$ for $0 \leq a \leq 1$ and $a_{*}=$ a for $a>1$. Let $\|\cdot\|_{a}=\|\cdot\|_{\mathcal{C}^{a}(D)}$. Then

$$
\begin{aligned}
\rho^{a_{*}}\left\|\prod_{j=1}^{m} u_{j}\right\|_{a} \leq C_{a, c_{0}} \sum_{j=1}^{m}\left\|u_{j}\right\|_{a} \prod_{i \neq j}\left\|u_{i}\right\|_{0}, \\
\rho^{e} \prod_{j=1}^{m}\|u\|_{d_{j}+b_{j}} \leq C_{a, b, c, c_{0}}\left(\prod_{j=1}^{m}\left\|u_{j}\right\|_{d_{j}+a_{j}}+\prod_{j=1}^{m}\left\|u_{j}\right\|_{d_{j}+c_{j}}\right),
\end{aligned}
$$

where $e=\left(b_{1}+d_{1}-\left[d_{1}\right]\right)+\cdots+\left(b_{m}+d_{m}-\left[d_{m}\right]\right), a_{j}, c_{j}, d_{j}$ are non-negative real numbers, and $\left(b_{1}, \ldots, b_{m}\right)$ is in the convex hull of $\left(a_{1}, \ldots, a_{m}\right)$ and $\left(c_{1}, \ldots, c_{m}\right)$. 
Proof. The first estimate is trivial if $a \leq 1$. So assume that $a>1$. We know that $D$ is convex and $B_{\rho / c_{0}} \subset D \subset B_{c_{0} \rho}$. Thus $D$ has the cone property if $1 \leq \rho \leq \rho_{0}$, where the cone of fixed size with vertex at $x \in \bar{D}$ can be found from the convex hull of $x$ and $B_{\rho / c_{0}}$. Assume now that $0<\rho<1$. Consider the isotropic dilation $S_{\rho}(x)=\rho x$. Then, $D_{*}=S_{\rho}^{-1} D$ has the cone property. Since $0<\rho<1$, we have

$$
\rho^{a}\|u\|_{a} \leq\left\|u \circ S_{\rho}\right\|_{D_{*}, a} \leq\|u\|_{a} .
$$

By Proposition A.4 (ii) we obtain

$$
\rho^{a}\left\|\prod_{j=1}^{m} u_{j}\right\|_{a} \leq\left\|\prod_{j=1}^{m} u_{j} \circ S_{\rho}\right\|_{D_{*}, a} \leq C \sum_{j=1}^{m}\left\|u_{j} \circ S_{\rho}\right\|_{D_{*}, a} \prod_{i \neq j}\left\|u_{i} \circ S_{\rho}\right\|_{D_{*}, 0} .
$$

Using (A.11), we get the first inequality easily. Let $l_{j} \leq\left[d_{j}\right]$ be any non-negative integers. By (A.11) and Proposition A.4 (iii), we get

$$
\begin{aligned}
& \rho^{e} \prod_{j=1}^{m}\left\|\partial^{l_{j}} u_{j}\right\|_{b_{j}+d_{j}-\left[d_{j}\right]} \leq \prod_{j=1}^{m}\left\|\left(\partial^{l_{j}} u_{j}\right) \circ S_{\rho}\right\|_{D_{*}, b_{j}+d_{j}-\left[d_{j}\right]} \\
& \quad \leq C\left(\prod_{j=1}^{m}\left\|\left(\partial^{l_{j}} u_{j}\right) \circ S_{\rho}\right\|_{D_{*}, a_{j}+d_{j}-\left[d_{j}\right]}+\prod_{j=1}^{m}\left\|\left(\partial^{l_{j}} u_{j}\right) \circ S_{\rho}\right\|_{D_{*}, c_{j}+d_{j}-\left[d_{j}\right]}\right) \\
& \quad \leq C\left(\prod_{j=1}^{m}\left\|u_{j}\right\|_{l_{j}+a_{j}+d_{j}-\left[d_{j}\right]}+\prod_{j=1}^{m}\left\|u_{j}\right\|_{l_{j}+c_{j}+d_{j}-\left[d_{j}\right]}\right) \\
& \quad \leq C\left(\prod_{j=1}^{m}\left\|u_{j}\right\|_{d_{j}+a_{j}}+\prod_{j=1}^{m}\left\|u_{j}\right\|_{d_{j}+c_{j}}\right) .
\end{aligned}
$$

Summing over all non-negative integers $l_{j} \leq\left[d_{j}\right]$ gives us the second inequality.

One can also obtain other inequalities via dilation. The inequalities below are not directly used in this paper.

Proposition A.6. Let $a_{*}$ be as in Proposition A.5. Let $\rho, D,\|\cdot\|_{a}$ be as in Proposition A.5. Let $a, b, a_{j}, b_{j}$ be nonnegative real numbers.

(i) $\rho^{c_{*}}\|u\|_{\lambda a+(1-\lambda) b} \leq C_{a, b}\|u\|_{a}^{\lambda}\|u\|_{b}^{1-\lambda}$ for $0<\lambda<1$, where $c_{*}=\lambda a+(1-$ $\lambda) b$.

(ii) Let $f$ be a map from $D$ into $D^{\prime} \subset \mathbb{R}^{n}$. Assume that $D^{\prime}$ is convex and $B_{c_{0}^{-1} \rho} \subset$ $D^{\prime} \subset B_{c_{0} \rho}$. Then

$$
\begin{gathered}
\rho^{a_{*}}\|u \circ f\|_{a} \leq C_{a, c_{0}} \rho\left(\left\|u^{\prime}\right\|_{D^{\prime}, a-1}\left\|f^{\prime}\right\|_{0}^{a}+\left\|u^{\prime}\right\|_{D^{\prime}, 0}\left\|f^{\prime}\right\|_{a-1}\right)+\|u\|_{D^{\prime}, 0}, \quad a \geq 1, \\
\|u \circ f\|_{a} \leq C_{c_{0}} \min \left(\|u\|_{D^{\prime}, 1}\|f\|_{a},\|u\|_{D^{\prime}, a}\|f\|_{1}^{a}\right)+\|u\|_{D^{\prime}, 0}, \quad 0 \leq a \leq 1 .
\end{gathered}
$$


(iii) Let $a_{* *}=0$ for $0 \leq a \leq 2$ and $a_{* *}=a-1$ for $a>2$. Let $f$ be a $\mathcal{C}^{a}$ map from $D$ into $\mathbb{R}^{m}$. There exists $C_{0}>1$ such that if $\left|f^{\prime}-I\right|<1 / C_{0}$ then

$$
\rho^{a_{* *}}\left\|f^{-1}-I\right\|_{f(D), a} \leq C_{a}\|f-I\|_{a} .
$$

(iv) Let $f=I+\tilde{f}$ be a $\mathcal{C}^{1}$ map from $D$ into $D^{\prime} \subset \mathbb{R}^{m}$ with $\left|f^{\prime}\right|<C_{0}$. Assume that $D \cup D^{\prime}$ is contained in a convex domain $D^{\prime \prime}$ satisfying $B_{\rho / c_{0}} \subset D^{\prime \prime} \subset B_{c_{0} \rho}$. Then

$$
\rho^{a_{*}}\|u \circ f-u\|_{a} \leq C_{a, c_{0}}\left(\left\|u^{\prime}\right\|_{D^{\prime \prime}, a}\|\tilde{f}\|_{0}+\left\|u^{\prime}\right\|_{D^{\prime \prime}, 0}\|\tilde{f}\|_{a}\right) .
$$

Proof. We may assume that $0<\rho<1$. Let $u_{*}(x)=u(\rho x), f^{*}(x)=\rho^{-1} f(\rho x)=$ $x+\tilde{f}^{*}(x)$, and $D_{*}=\rho^{-1} D$. Then $D_{*}$ has the cone property.

(i) is immediate, by applying Proposition A.4 to $u_{*}$ and by using (A.11).

(ii). The case $0 \leq a \leq 1$ is verified directly. Assume that $a>1$. Applying Proposition A.4 to $u_{*} \circ f^{*}$, we get

$\left\|u_{*} \circ f^{*}\right\|_{D_{*}, a} \leq C_{a}\left(\left\|\partial u_{*}\right\|_{D_{*}^{\prime}, a-1}\left\|f^{* \prime}\right\|_{D_{*}, 0}^{a}+\left\|\partial u_{*}\right\|_{D_{*}^{\prime}, 0}\left\|f^{* \prime}\right\|_{D_{*}, a-1}\right)+\left\|u_{*}\right\|_{D_{*}^{\prime}, 0}$.

Since $f^{* \prime}(x)=f^{\prime}(\rho x)$, then $\left\|f^{* \prime}\right\|_{D_{*}, b} \leq\left\|f^{\prime}\right\|_{D, b}$ for $b \geq 0$. By (A.11), we also have

$$
\begin{gathered}
\left\|u_{*} \circ f^{*}\right\|_{D_{*}, a}=\left\|(u \circ f) \circ S_{\rho}\right\|_{D_{*}, a} \geq \rho^{a}\|u \circ f\|_{a}, \\
\left\|\partial u_{*}\right\|_{D_{*}^{\prime}, a-1}=\left\|\rho(\partial u)_{*}\right\|_{D_{*}^{\prime}, a-1} \leq \rho\|\partial u\|_{a-1}, \quad a \geq 1 .
\end{gathered}
$$

Simplifying gives us (ii).

(iv). Let $\tilde{f}=f-I$. The case $0 \leq a \leq 1$ is verified directly. Assume that $a>1$. Then

$$
\left\|u_{*} \circ f^{*}-u_{*}\right\|_{D_{*}, a} \leq C\left(\left\|\tilde{f}^{*}\right\|_{a}\left\|\partial u_{*}\right\|_{D_{*}^{\prime \prime}, 0}+\left\|\tilde{f}^{*}\right\|_{0}\left\|\partial u_{*}\right\|_{D_{*}^{\prime \prime}, a}\right) .
$$

As in (ii), we can get (iv) by (A.11) and

$$
\left\|\tilde{f}^{*}\right\|_{D_{*}^{\prime}, a}=\rho^{-1}\left\|\tilde{f} \circ S_{\rho}\right\|_{D_{*}^{\prime}, a} \leq \rho^{-1}\|\tilde{f}\|_{a} .
$$

(iii). (A dilation would give us $a_{* *}=a$.) Let $g=f^{-1}=I+\tilde{g}$. We have $\tilde{g}=-\tilde{f} \circ g$ and $\tilde{g}^{\prime}=-\left\{\tilde{f}^{\prime}\left(\mathbf{1}+\tilde{f}^{\prime}\right)^{-1}\right\} \circ g$. By Lemma A.1, $C^{-1}\left|x^{\prime}-x\right| \leq$ $\left|g\left(x^{\prime}\right)-g(x)\right| \leq C\left|x^{\prime}-x\right|$. We get immediately

$$
\|\tilde{g}\|_{f(D), a} \leq C\|\tilde{f}\|_{a}, \quad 0 \leq a \leq 2 .
$$

Assume that $k \geq 1$. Recall that by (A.7)-(A.8),

$\partial^{K} \tilde{g}=\sum_{j_{1}+\cdots+j_{l} \leq k}\left\{p\left(\tilde{f}^{\prime}\right) \partial^{J_{1}} \tilde{f} \cdots \partial^{J_{l}} \tilde{f}\right\} \circ g, \quad k=|K|-1 \geq 0, j_{i}=|J|_{i}-1 \geq 0$.

Set $1+k=[a]$ and $\alpha=a-k-1$. Computing the Hölder ratio of (A.8) and applying Proposition A.5 with $d_{j}=1$, we obtain (iii) from

$$
\left\|\partial^{K} \tilde{g}\right\|_{\alpha} \leq C \sum_{j_{1}+\cdots+j_{l} \leq k} \rho^{-j_{1}-\cdots-j_{l}-\alpha}\|\tilde{f}\|_{1+k+\alpha} \leq C^{\prime} \rho^{-k-\alpha}\|\tilde{f}\|_{1+k+\alpha} .
$$


Remark A.7. If all norms in Proposition A.6 are replaced by the scalar-invariant norm $\|\cdot\|^{*}$, defined as

$$
\|u\|_{D, a}^{*}=\left\|u \circ S_{d}\right\|_{S_{d}^{-1} D, a}
$$

with $d$ being the diameter of $D$, we can take $c_{*}=a_{*}=a_{* *}=0$ for the proposition. For the use of scalar-invariant norms to derive estimates on the Bochner-MartinelliKoppelman formula for balls in $\mathbb{C}^{n}$, see [20].

\section{B. The Henkin homotopy formula}

Recall notation $z^{\prime}=\left(z_{1}, \ldots, z_{n-1}\right), z=\left(z^{\prime}, z^{n}\right)$ and $x=\pi(z)=\left(\operatorname{Re} z, \operatorname{Im} z^{\prime}\right)$. formula.

In this appendix, we will derive the following version of Henkin's homotopy

Theorem B.1. Let $M \subset \mathbb{C}^{n}$ be a graph $y^{n}=\left|z^{\prime}\right|^{2}+\hat{r}(x)$ over $D \subset \mathbb{R}^{2 n-1}$. Assume that $0<\rho<\rho_{0} \leq 3$ and

$$
\overline{D_{\rho_{0}}} \subset D, \quad \hat{r}(0)=0, \quad \partial \hat{r}(0)=0, \quad\|\hat{r}\|_{\rho_{0}, 2}=\|\hat{r}\|_{D_{\rho_{0}}, 2}<1 / C_{0}
$$

with $C_{0}$ sufficiently large. Assume that $0<\rho<\rho_{0} \leq 3$. Let $\varphi$ be a continuous tangential $(0, q)$-form on $\bar{M}_{\rho}$. Assume that $\bar{\partial}_{M} \varphi$ is continuous as currents on $M_{\rho}$ and admits a continuous extension on $\bar{M}_{\rho}$. If $0<q<n-2$, then on $M_{\rho}$ and as currents

$$
\varphi=\bar{\partial}_{M}\left(P_{0}+P_{1}\right) \varphi+\left(Q_{0}+Q_{1}\right) \bar{\partial}_{M} \varphi \bmod \bar{\partial} r
$$

where $P_{0}, P_{1}, Q_{0}, Q_{1}$ are defined by (3.5).

Recall that $M_{\rho}=M \cap\left\{z:\left|x^{n}\right|^{2}+y^{n}<\rho^{2}\right\}$ and $D_{\rho}=\left\{x \in D:\left|x^{n}\right|^{2}+y^{n}<\right.$ $\left.\rho^{2}\right\}$. When $M$ is strictly convex, see Henkin [7] for the proof. Our proof will follow [21] via Stokes' theorem.

Set $r=-y^{n}+\left|z^{\prime}\right|^{2}+\hat{r}(x)$ and define

$$
\begin{gathered}
F(\zeta, z)=r_{z} \cdot(\zeta-z)+\frac{1}{2} \sum_{1 \leq j, k \leq n} r_{z^{j} z^{k}}\left(\zeta^{j}-z^{j}\right)\left(\zeta^{k}-z^{k}\right), \\
S_{t}(z)=\pi\{\zeta \in M:|F(\zeta, z)|=t\}, \quad S_{t}^{\prime}(\zeta)=\pi\{z \in M:|F(\zeta, z)|=t\} .
\end{gathered}
$$

Note that $r_{z_{j} z_{k}}=\hat{r}_{z_{j} z_{k}}$.

Lemma B.2. Let $n \geq 2$. Let $M$ be as in Theorem B.1. There exists $C_{1}>1$ satisfying the following.

(i) If $0<t<\left(\rho_{0}-\rho\right)^{2} / C_{1}$ and $z \in M_{\rho}$, then $S_{t}(z)$ and $S_{t}^{\prime}(z)$ are compact subsets of $D_{\rho_{0}}$.

(ii) Assume that $\hat{r} \in \mathcal{C}^{3}\left(\bar{D}_{\rho_{0}}\right), z \in M_{\rho}$ and $0<t<C_{1}^{-1} \min \left\{\left(\rho_{0}-\rho\right)^{2},(1+\right.$ $\left.\left.\|\hat{r}\|_{\rho_{0}, 3}\right)^{-1}\right\}$. Then $S_{t}(z)$ and $S_{t}^{\prime}(z)$ are smooth and of classes $\mathcal{C}^{3}$ and $\mathcal{C}^{1}$, respectively. 
Proof. Set $\epsilon=\|\hat{r}\|_{\rho_{0}, 2}$. (i) By Lemma 5.1, $D_{\rho_{0}}$ is convex and $\operatorname{dist}\left(\partial D_{\rho_{0}}, \partial D_{\rho}\right) \geq$ $\left(\rho_{0}-\rho\right) / C$. On $M_{\rho_{0}} \times M_{\rho_{0}},\left|r_{z} \cdot(\zeta-z)\right| \geq|\zeta-z|^{2} / C$ by Lemma 6.3 and $\left|\hat{r}_{z^{j} z^{k}}\right| \leq C \epsilon$. Hence, (i) follows from

$$
|F(\zeta, z)| \geq|\zeta-z|^{2} /(2 C) .
$$

(ii) Fix $z \in M_{\rho_{0}}$. Let $\zeta_{*}^{n}=-2 i r_{z}(\zeta-z)$. By Lemma $6.1, \xi_{*}=\left(\operatorname{Re} \zeta_{*}, \operatorname{Im} \zeta_{*}^{\prime}\right)$ are coordinates of $M_{\rho_{0}}$ and

$$
\begin{aligned}
& \xi_{*}^{n}=\operatorname{Re}\left(-2 i r_{z} \cdot(\zeta-z)\right)=\xi^{n}-x^{n}+2 \operatorname{Im}\left(\overline{z^{\prime}} \cdot \zeta^{\prime}\right)+\operatorname{Im}\left\{2 \hat{r}_{z} \cdot(\zeta-z)\right\} \\
& \eta_{*}^{n}=\operatorname{Im}\left(-2 i r_{z} \cdot(\zeta-z)\right)=\left|\zeta_{*}^{\prime}\right|^{2}+\sum_{|I|=2} \mathcal{R}_{*}^{2} \hat{r}(\xi, x) \xi_{*}^{I},
\end{aligned}
$$

where $\mathcal{R}_{*}^{i}$ is defined by (6.5). Write $u=\operatorname{Re}(-2 i F(\zeta, z))$ and $v=\operatorname{Im}(-2 i F(\zeta, z))$. We have

$$
\begin{gathered}
-2 i F(\zeta, z)=-2 i r_{z} \cdot(\zeta-z)-i \sum_{1 \leq j, k \leq n} r_{z^{j} z^{k}}\left(\zeta^{j}-z^{j}\right)\left(\zeta^{k}-z^{k}\right), \\
u\left(\xi_{*}\right)=\xi_{*}^{n}+\tilde{u}\left(\xi_{*}\right), \quad \tilde{u}\left(\xi_{*}\right)=\sum_{|I|=2} a_{I} \circ \Psi^{-1}\left(\xi_{*}, x\right) \xi_{*}^{I}, \\
v\left(\xi_{*}\right)=\left|\zeta_{*}^{\prime}\right|^{2}+\tilde{v}\left(\xi_{*}\right), \quad \tilde{v}\left(\xi_{*}\right)=\sum_{|I|=2} b_{I} \circ \Psi^{-1}\left(\xi_{*}, x\right) \xi_{*}^{I},
\end{gathered}
$$

where $\left|a_{I}\right|+\left|b_{I}\right| \leq C\|\hat{r}\|_{\rho_{0}, 2} \leq C^{\prime} \epsilon$ and $\left|\partial^{1} a_{I}(\zeta, z)\right|+\left|\partial^{1} b_{I}(\zeta, z)\right| \leq C\|\hat{r}\|_{\rho_{0}, 3}$. Suppose that $|F(\zeta, z)|=t$ and

$$
t^{1 / 2}\|\hat{r}\|_{\rho_{0}, 3}<1 / C_{0}
$$

and $C_{0}$ is sufficiently large. To show that $S_{t}(z)$ is smooth, we need to verify that $d_{\zeta_{*}^{\prime}, \xi_{*}^{n}}\left(u^{2}+v^{2}\right) \neq 0$ when $u^{2}+v^{2}=t^{2}$. By (B.3)-(B.4) we know that

$$
|\zeta-z| / C \leq\left|\xi_{*}\right| \leq C|\zeta-z| \leq C^{\prime} t^{1 / 2}
$$

By (B.3) and (B.8), we obtain $|\zeta-z|\|\hat{r}\|_{\rho_{0}, 3} \leq 1 / C_{0}$ and

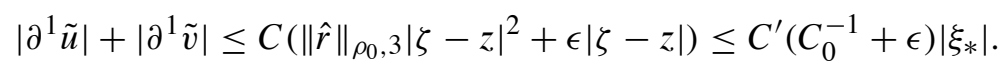

Assume that $d_{\zeta_{*}^{\prime}, \xi_{*}^{n}}\left(u^{2}+v^{2}\right)=0$. Then

$$
\left(\xi_{*}^{n}+\tilde{u}\right)\left(1+\tilde{u}_{\xi_{*}^{n}}\right)+v v_{\xi_{*}^{n}}=0, \quad u \tilde{u}_{\zeta_{*}^{\prime}}+v\left(\bar{\zeta}_{*}^{\prime}+\tilde{v}_{\zeta_{*}^{\prime}}\right)=0 .
$$

From the first identity we get $\left|\xi_{*}^{n}\right| \leq C(|\tilde{u}|+|v|) \leq C^{\prime}\left(\left|\zeta_{*}^{\prime}\right|^{2}+\left|\xi_{*}^{n}\right|^{2}\right)$. Therefore, for $t<1 / C,\left|\xi_{*}^{n}\right| \leq C\left|\zeta_{*}^{\prime}\right|^{2}$. Now, $|v| \geq\left|\zeta_{*}^{\prime}\right|^{2}-C \epsilon\left(\left|\zeta_{*}^{\prime}\right|^{2}+\left|\xi_{*}^{n}\right|^{2}\right) \geq \frac{1}{2}\left|\zeta_{*}^{\prime}\right|^{2}$ and $|u| \leq C\left|\zeta_{*}^{\prime}\right|^{2}$. By Lemma 5.2, $\left|\partial_{\xi_{*}}^{1} \xi\right| \leq C$, and by (B.7) and (B.8),

$$
\begin{gathered}
\left|\tilde{v}_{\zeta_{*}}\right| \leq\left|\zeta_{*}^{\prime}\right| / 2, \quad\left|\overline{\zeta_{*}^{\prime}}+\tilde{v}_{\zeta_{*}^{\prime}}\right| \geq\left|\zeta_{*}^{\prime}\right| / 2, \\
\left|\zeta_{*}^{\prime}\right|^{3} / 4 \leq \mid v\left(\overline{\zeta_{*}^{\prime}}+\left.\tilde{v}_{\zeta_{*}^{\prime}}|=| u \tilde{u}_{\zeta_{*}^{\prime}}|\leq C| \zeta_{*}^{\prime}\right|^{2}\left(\epsilon+C_{0}^{-1}\right)\left|\zeta_{*}^{\prime}\right| .\right.
\end{gathered}
$$


Hence $\zeta_{*}^{\prime}=\xi_{*}^{n}=0$, when $C_{0}^{-1}, \epsilon$ are sufficiently small. This shows that $t=0$, a contradiction. It is clear that for a fixed $z, u^{2}+v^{2}$ is a function of class $\mathcal{C}^{3}$ in $\left(\zeta^{\prime}, \xi^{n}\right)$. This shows that $S_{t}(z)$ is smooth and of class $\mathcal{C}^{3}$.

For a fixed $\zeta \in M_{\rho},\left(\zeta_{*}^{\prime}, \xi_{*}^{n}\right)$ still form a coordinate system of class $\mathcal{C}^{2}$ for $M$. We have the same formulae (B.4)-(B.5), only to vary $z \in M_{\rho_{0}}$; see Remark 6.2. Thus (B.6)-(B.7) are still valid, where $\xi$ is fixed and $z$ varies in $M_{\rho_{0}}$. The same argument shows that $S_{t}^{\prime}(\zeta)$ is smooth and of class $\mathcal{C}^{1}$.

Let us recall from Section 3

$$
\Omega_{0, q}^{+-}=\frac{\left(r_{\zeta}-r_{z}\right) \cdot d \zeta \wedge r_{z} \cdot d \zeta \wedge\left(\bar{\partial}_{\zeta} \partial_{\zeta} r\right)^{n-2-q} \wedge\left(\bar{\partial}_{z} r_{z} \wedge d \zeta\right)^{q}}{\left(r_{\zeta} \cdot(\zeta-z)\right)^{n-1-q}\left(r_{z} \cdot(\zeta-z)\right)^{q+1}}
$$

The following computation is essential in Folland-Stein [3]. See also Romero [17].

Lemma B.3. Let $n \geq 2$ and $0 \leq q \leq n-1$. Let $M$ satisfy (B.1) and $\hat{r} \in \mathcal{C}^{3}$. Let $F(\zeta, z)$ be the Levi polynomial of $r$ about $z \in M$. Let $\varphi$ be a continuous tangential $(0, q)$-form on $M$. Then

$$
\lim _{t \rightarrow 0} \int_{|F(\zeta, z)|=t, \zeta \in M} \varphi(\zeta) \wedge \Omega_{0, q}^{+-}(\zeta, z)=\frac{(2 \pi i)^{n}}{2} \varphi(z) \bmod \bar{\partial} r(z),
$$

where the convergence is uniformly in $z$ on each compact subset of $M$.

In the lemma, $|F(z, \cdot)|=t\}$ is oriented as the boundary of the domain $|F(z, \cdot)|<t$ in $M$ on which $d V=d \xi^{1} \wedge d \eta^{1} \wedge \cdots \wedge d \eta^{n-1} \wedge d \xi^{n}$ is the volume-form.

Here is an outline. Following [3], we will use the Levi polynomial $F(\zeta, z)$ to define new coordinates of $M$ near $z$ and compute each term in the kernel. On $T_{z}^{1,0} M$ there are two quadratic forms $h=\sum_{1 \leq j, k \leq n} r_{z^{j} z^{k}} t^{j} \bar{t}^{k}, A=\sum_{1 \leq j, k \leq n} r_{z^{j} z^{k}} t^{j} t^{k}$. We will express the kernel in $h$ and $A$, with error terms. When we compute the residue, the error terms can be removed via non-isotropic dilation. This gives us a limit kernel (see (B.19) below) on a non-isotropic sphere. Roughly speaking, the limit kernel is expressed in $h$ and $A$, but not in $\bar{A}$. For latter purpose we will use $-2 i F(\zeta, z)$, instead of its linear part $-2 i r_{z} \cdot(\zeta-z)$, as part of coordinates. Without $\bar{A}$, we remove $A$ in the limit kernel by averaging. By a linear transformation, we reduce $h$ to the identity, and compute the residue.

CHANGE NOTATION. Let $\zeta_{*}^{\prime}=\zeta^{\prime}-z^{\prime}$ and

$$
\zeta_{*}^{n}=-2 i F(\zeta, z)=-2 i r_{z} \cdot(\zeta-z)-i \sum_{1 \leq j, k \leq n} \hat{r}_{z^{j} z^{k}}\left(\zeta^{j}-z^{j}\right)\left(\zeta^{k}-z^{k}\right) .
$$

Then near $z \in M_{\rho_{0}}, \zeta_{*}^{\prime}, \xi_{*}^{n}$ form coordinates of $M_{\rho_{0}}$. We will modify some earlier computations where the approximate Heisenberg transformation is used. 
Applying the Taylor formula on convex domain $D_{\rho_{0}} \times \mathbb{R}$ and then letting $\zeta, z \in$ $M_{\rho_{0}}$, we obtain

$$
\begin{gathered}
r(\zeta)-r(z)=2 \operatorname{Re} F(\zeta, z)+\sum_{1 \leq j, k \leq n} r_{z^{j}} \overline{z^{k}}\left(\zeta^{j}-z^{j}\right)\left(\overline{\zeta^{k}-z^{k}}\right)+E(\zeta, z), \\
E(\zeta, z)=o\left(\left|\left(\zeta^{\prime}-z^{\prime}, \xi^{n}-x^{n}\right)\right|^{2}\right), \quad\left|\partial^{1} E(\zeta, z)\right| \leq C\|\hat{r}\|_{\rho_{0}, 3}\left|\left(\zeta^{\prime}-z^{\prime}, \xi^{n}-x^{n}\right)\right|^{2} .
\end{gathered}
$$

With $z \in M_{\rho_{0}}$ the equation $r(\zeta)=0$ becomes

$$
-\eta_{*}^{n}+\sum_{1 \leq j, k \leq n} r_{z^{j}} \bar{z}^{k}\left(\zeta^{j}-z^{j}\right)\left(\overline{\zeta^{k}-z^{k}}\right)+E(\zeta, z)=0
$$

where $\zeta_{n}-z_{n}$, with $\zeta^{\prime}-z^{\prime}=\zeta_{*}$, is a local solution to (B.10). Replace $\zeta-z$ by the solution expressed in $\zeta_{*}$. Solving for $\eta_{*}^{n}$ from the new equation shows that for $\left(\zeta_{*}^{\prime}, \xi_{*}^{n}\right) \in \pi \psi_{z}\left(M_{\rho_{0}}\right)$

$$
\eta_{*}^{n}=\sum_{1 \leq \alpha, \beta<n} h_{\alpha \bar{\beta}} \zeta_{*}^{\alpha} \bar{\zeta}_{*}^{\beta}+o\left(\left|\zeta_{*}^{\prime}\right|^{2}\right)+O\left(\left|\xi_{*}^{n}\right|\left|\zeta_{*}^{\prime}\right|+\left|\xi_{*}^{n}\right|^{2}\right)
$$

Write $A\left(\zeta_{*}^{\prime}, \xi_{*}^{n}\right)=o(k)$ if $t^{-k} A\left(t \zeta_{*}^{\prime}, t^{2} \xi_{*}^{n}\right)$ tends to zero uniformly for $\left|\zeta_{*}^{\prime}\right|+\left|\xi_{*}^{n}\right|<1$ and small $|t|$, where $A$ is a function or differential form. Write $A\left(\zeta_{*}^{\prime}, \xi_{*}^{n}\right)=O(k)$ if $\left|t^{-k} A\left(t \zeta_{*}^{\prime}, t^{2} \xi_{*}^{n}\right)\right| \leq C$. Thus $\zeta^{\prime}-z^{\prime}=O(1), \zeta_{*}^{n}=O(2), d \xi_{*}^{\prime}=O(1)$ and $d \xi_{*}^{n}=O(2)$. By (B.10)-(B.11), $\zeta^{n}-z^{n}=-\frac{r_{z^{\prime}}}{r_{z^{n}}} \cdot \zeta_{*}^{\prime}+O(2)$. The Levi matrix $\left(h_{\alpha \bar{\beta}}\right)$ at $z$ is determined by

$$
\sum_{1 \leq j, k \leq n} r_{z^{j}} \overline{z^{k}}\left(\zeta^{j}-z^{j}\right)\left(\overline{\zeta^{k}-z^{k}}\right)=\sum_{1 \leq \alpha, \beta<n} h_{\alpha \bar{\beta}} \zeta_{*}^{\alpha} \bar{\zeta}_{*}^{\beta}+o(2) .
$$

Explicitly, we have

$$
h_{\alpha \bar{\beta}}=r_{z^{\alpha} \overline{z^{\beta}}}-r_{z^{\alpha} \overline{z^{n}}} \frac{r \overline{z^{\beta}}}{r_{\overline{z^{n}}}}-\frac{r_{z^{\alpha}}}{r_{z^{n}}} r_{z^{n} \overline{z^{\beta}}}+r_{z^{n}} \overline{z^{n}} \frac{r_{z^{\alpha}}}{r_{z^{n}}} \frac{r \overline{z^{\beta}}}{r \overline{z^{n}}} .
$$

We also have the holomorphic quadratic form

$$
\sum_{1 \leq j, k \leq n} r_{z^{j} z^{k}}\left(\zeta^{j}-z^{j}\right)\left(\zeta^{k}-z^{k}\right)=\sum_{1 \leq \alpha, \beta<n} A_{\alpha \beta} \zeta_{*}^{\alpha} \zeta_{*}^{\beta}+o(2) .
$$

Here $A_{\alpha \beta}=A_{\beta \alpha}$. Set $h=\left(h_{\alpha \bar{\beta}}\right)$ and $A=\left(A_{\alpha \beta}\right)$. To simplify notation, we define

$$
h\left(\zeta_{*}^{\prime}, \overline{\zeta_{*}^{\prime}}\right)=\sum_{1 \leq \alpha, \beta<n} h_{\alpha \bar{\beta}} \zeta_{*}^{\alpha} \bar{\zeta}_{*}^{\beta}, \quad A\left(\zeta_{*}^{\prime}, \zeta_{*}^{\prime}\right)=\sum_{1 \leq \alpha, \beta<n} A_{\alpha \beta} \zeta_{*}^{\alpha} \zeta_{*}^{\beta}
$$

We can write

$$
\bar{\partial}_{\zeta_{*}} h\left(\zeta_{*}^{\prime}, \overline{\zeta_{*}^{\prime}}\right)=h\left(\zeta_{*}^{\prime}, d \overline{\zeta_{*}^{\prime}}\right), \quad \partial_{\zeta_{*}} A\left(\zeta_{*}^{\prime}, \zeta_{*}^{\prime}\right)=2 A\left(d \zeta_{*}^{\prime}, \zeta_{*}^{\prime}\right)
$$


We will need to express the kernel in $h=\left(h_{\alpha \bar{\beta}}\right)$ and $A=\left(A_{\alpha \beta}\right)$, but not in $\left(\bar{A}_{\alpha \beta}\right)$. This will play a crucial role, when we eliminate $A$ later via averaging.

For the denominator of the kernel, using (B.10)-(B.11) we get

$$
\begin{aligned}
-2 i r_{z} \cdot(\zeta-z) & =\zeta_{*}^{n}+i \sum_{1 \leq j, k \leq n} r_{z^{j} z^{k}}\left(\zeta^{j}-z^{j}\right)\left(\zeta^{k}-z^{k}\right) \\
& =\xi_{*}^{n}+i\left(h\left(\zeta_{*}^{\prime}, \overline{\zeta_{*}^{\prime}}\right)+A\left(\zeta_{*}^{\prime}, \zeta_{*}^{\prime}\right)\right)+o(2) .
\end{aligned}
$$

Using $r_{\zeta} \cdot(\zeta-z)=r_{z} \cdot(\zeta-z)+\left(r_{\zeta}-r_{z}\right) \cdot(\zeta-z)$, we get

$$
\begin{aligned}
& -2 i r_{\zeta} \cdot(\zeta-z)=-2 i r_{z} \cdot(\zeta-z)-2 i \sum_{1 \leq j, k \leq n} r_{z^{j} z^{k}}\left(\zeta^{j}-z^{j}\right)\left(\zeta^{k}-z^{k}\right) \\
& \quad-2 i \sum_{1 \leq j, k \leq n} r_{z^{j}} \bar{z}^{k}\left(\zeta^{j}-z^{j}\right)\left(\overline{\zeta^{k}-z^{k}}\right)+o(2) \\
& =-2 i r_{z} \cdot(\zeta-z)-2 i\left(h\left(\zeta_{*}^{\prime}, \overline{\zeta_{*}^{\prime}}\right)+A\left(\zeta_{*}^{\prime}, \zeta_{*}^{\prime}\right)\right)+o(2) \quad(\text { by }(\mathrm{B} .12),(\mathrm{B} .14)) \\
& =\xi_{*}^{n}-i\left(h\left(\zeta_{*}^{\prime}, \overline{\zeta_{*}^{\prime}}\right)+A\left(\zeta_{*}^{\prime}, \zeta_{*}^{\prime}\right)\right)+o(2) . \quad(\text { by }(\mathrm{B} .15))
\end{aligned}
$$

We arrive at the basic relations

$$
\begin{aligned}
& -2 i r_{\zeta} \cdot(\zeta-z)=\xi_{*}^{n}-i\left(h\left(\zeta_{*}^{\prime}, \overline{\zeta_{*}^{\prime}}\right)+A\left(\zeta_{*}^{\prime}, \zeta_{*}^{\prime}\right)\right)+o(2), \\
& -2 i r_{z} \cdot(\zeta-z)=\xi_{*}^{n}+i\left(h\left(\zeta_{*}^{\prime}, \overline{\zeta_{*}^{\prime}}\right)+A\left(\zeta_{*}^{\prime}, \zeta_{*}^{\prime}\right)\right)+o(2)
\end{aligned}
$$

We now computer the numerator of the kernel. Using the first-order expansion of $r_{\zeta}$ about $z$, we get

$$
\begin{aligned}
\left(r_{\zeta}-r_{z}\right) \cdot d \zeta= & \sum_{1 \leq j, k \leq n}\left\{r_{z^{j}} z^{k}\left(\zeta^{k}-z^{k}\right)+r_{z^{j}} \overline{z^{k}}\left(\overline{\zeta^{k}-z^{k}}\right)+O(2)\right\} d \zeta^{j} \\
= & \partial_{\zeta} \sum_{1 \leq j, k \leq n}\left\{\frac{1}{2} r_{z^{j} z^{k}}\left(\zeta^{j}-z^{j}\right)\left(\zeta^{k}-z^{k}\right)+r_{z^{j}} \overline{z^{k}}\left(\zeta^{j}-z^{j}\right)\left(\overline{\zeta^{k}-z^{k}}\right)\right\} \\
& +o(2) .
\end{aligned}
$$

Note that for fixed $z, \zeta \rightarrow \zeta_{*}$ is holomorphic. So we can switch the above $\partial_{\zeta}$ to $\partial_{\zeta_{*}}$ and then restrict it to $M$, which gives us

$$
\left(r_{\zeta}-r_{z}\right) \cdot d \zeta=h\left(d \zeta_{*}^{\prime}, \overline{\zeta_{*}^{\prime}}\right)+A\left(d \zeta_{*}^{\prime}, \zeta_{*}^{\prime}\right)+o(2)
$$

Recall that $\zeta_{*}^{n}=-2 i r_{z} \cdot(\zeta-z)-i \sum_{1 \leq j, k \leq n} r_{z^{j} z^{k}}\left(\zeta^{j}-z^{j}\right)\left(\zeta^{k}-z^{k}\right)$. Applying $\partial_{\zeta}$ gives us

$$
\begin{aligned}
-2 i r_{z} \cdot d \zeta & =\partial_{\zeta}\left\{\zeta_{*}^{n}+i \sum_{1 \leq j, k \leq n} r_{z^{j} z^{k}}\left(\zeta^{j}-z^{j}\right)\left(\zeta^{k}-z^{k}\right)\right\} \\
& =\partial_{\zeta}\left\{\zeta_{*}^{n}+i \sum_{1 \leq \alpha, \beta<n} A_{\alpha \beta} \zeta_{*}^{\alpha} \zeta_{*}^{\beta}+o(2)\right\} \quad \text { by } \\
& =d \zeta_{*}^{n}+2 i A\left(d \zeta_{*}^{\prime}, \zeta_{*}^{\prime}\right)+o(2) .
\end{aligned}
$$


Recall that $z$ is fixed. So on $M$ we have

$$
\begin{aligned}
& \bar{\partial}_{\zeta} \partial_{\zeta} r=\bar{\partial} \partial r(\zeta)=\bar{\partial} \partial\left\{\sum_{1 \leq j, k \leq n} r_{z^{j}} \bar{z}^{k}\left(\zeta^{j}-z^{j}\right)\left(\overline{\zeta^{k}-z^{k}}\right)+o(2)\right\} \\
& =\bar{\partial} \partial\left\{\sum_{1 \leq \alpha, \beta<n} h_{\alpha \bar{\beta}} \zeta_{*}^{\alpha} \bar{\zeta}_{*}^{\beta}\right\}+o(2) \quad \text { by (B.12) } \\
& =-h\left(d \zeta_{*}^{\prime}, d \overline{\zeta_{*}^{\prime}}\right)+o(2) \text {. }
\end{aligned}
$$

On $M, \bar{\partial} r_{z^{j}}=\bar{\partial} M_{z^{j}} \bmod \bar{\partial} r(z)$ and $\bar{\partial} r_{z^{j}}=\sum_{\beta=1}^{n-1}\left(r_{z^{j} \bar{z}^{\beta}}-\frac{r_{\bar{z} \beta}}{r_{\bar{z} n} n} r_{z^{j} \bar{z}^{n}}\right) d \bar{z}^{\beta} \bmod \bar{\partial} r(z)$. Recall that $\zeta^{n}-z^{n}=-r_{z^{n}}^{-1} r_{z^{\prime}} \cdot \zeta_{*}^{\prime}+O(2)$. Thus $d \zeta^{n}=-\frac{r_{z^{\prime}}}{r_{z}^{n}} \cdot d \zeta_{*}^{\prime}+O(2)$. Therefore,

$$
\begin{aligned}
\left(\bar{\partial} r_{z}\right) \wedge d \zeta= & \sum_{1 \leq \alpha, \beta<n}\left(r_{z^{\alpha} \bar{z}^{\beta}}-\frac{r_{\bar{z}^{\beta}}}{r_{\bar{z}^{n}}} r_{z^{\alpha} \bar{z}^{n}}\right) d \bar{z}^{\beta} \wedge d \zeta_{*}^{\alpha}+O(2) \\
& -\sum_{1 \leq \alpha, \beta<n}\left(r_{z^{n} \bar{z}^{\beta}}-\frac{r_{\bar{z}^{\beta}}}{r_{\bar{z}^{n}}} r_{z^{n} \bar{z}^{n}}\right) d \bar{z}^{\beta} \wedge \frac{r_{z^{\alpha}}}{r_{z^{n}}} d \zeta_{*}^{\alpha} \quad \bmod \bar{\partial} r(z)
\end{aligned}
$$

Looking at (B.13), we see that

$$
\left(\partial r_{z}\right) \wedge d \zeta=-h\left(d \zeta_{*}^{\prime}, d \overline{z^{\prime}}\right)+O(2) \bmod \bar{\partial} r(z)
$$

We apply the non-isotropy dilation $T_{t}\left(\zeta_{*}^{\prime}, \xi_{*}^{n}\right)=\left(t \zeta_{*}^{\prime}, t^{2} \xi_{*}^{n}\right)$ and summarize the above as

$$
\begin{aligned}
t^{-2} T_{t}^{*}\left\{-2 i r_{\zeta} \cdot(\zeta-z)\right\} & =\xi_{*}^{n}-i\left(h\left(\zeta_{*}^{\prime}, \overline{\zeta_{*}^{\prime}}\right)+A\left(\zeta_{*}^{\prime}, \zeta_{*}^{\prime}\right)\right)+o\left(t^{0}\right), \\
t^{-2} T_{t}^{*}\left\{-2 i r_{z} \cdot(\zeta-z)\right\} & =\xi_{*}^{n}+i\left(h\left(\zeta_{*}^{\prime}, \overline{\zeta_{*}^{\prime}}\right)+A\left(\zeta_{*}^{\prime}, \zeta_{*}^{\prime}\right)\right)+o\left(t^{0}\right), \\
t^{-2} T_{t}^{*}\left\{\left(r_{\zeta}-r_{z}\right) \cdot d \zeta\right\} & =h\left(d \zeta_{*}^{\prime}, \overline{\zeta_{*}^{\prime}}\right)+A\left(d \zeta_{*}^{\prime}, \zeta_{*}^{\prime}\right)+o\left(t^{0}\right), \\
t^{-2} T_{t}^{*}\left\{-2 i r_{z} \cdot d \zeta\right\} & =d \zeta_{*}^{n}+2 i A\left(d \zeta_{*}^{\prime}, \zeta_{*}^{\prime}\right)+o\left(t^{0}\right), \\
t^{-2} T_{t}^{*}\left\{\bar{\partial}_{\zeta} r_{\zeta} \dot{\wedge} d \zeta\right\} & =-h\left(d \zeta_{*}^{\prime}, d \overline{\zeta_{*}^{\prime}}\right)+o\left(t^{0}\right), \\
t^{-1} T_{t}^{*}\left\{\bar{\partial}_{z} r_{z} \dot{\wedge} d \zeta\right\} & =-h\left(d \zeta_{*}^{\prime}, d \overline{z^{\prime}}\right)+O(t) \quad \bmod \bar{\partial} r(z) .
\end{aligned}
$$

By (B.10) and (B.15), the subset of $M$ defined by $2|F(\zeta, z)|=t^{2}$ has the form

$$
S_{t}(z)=\left\{\left(\zeta_{*}^{\prime}, \xi_{*}^{n}\right):\left|\xi_{*}^{n}+i h\left(\zeta_{*}^{\prime}, \overline{\zeta_{*}^{\prime}}\right)+o(2)\right|=t^{2}\right\}
$$

Then we have a non-isotropic sphere

$$
\lim _{t \rightarrow 0} T_{t}^{-1}\left(S_{t}\right)=\left\{\left(\zeta_{*}^{\prime}, \xi_{*}^{n}\right):\left|\xi_{*}^{n}+i h\left(\zeta_{*}^{\prime}, \overline{\zeta_{*}^{\prime}}\right)\right|=1\right\} \stackrel{\text { def }}{=} S
$$


Set $N_{*}\left(\zeta_{*}^{\prime}, \xi_{*}^{n}\right)=\xi_{*}^{n}+i h\left(\zeta_{*}^{\prime}, \overline{\zeta_{*}^{\prime}}\right)$. As $t$ tends to $0, t^{q} T_{t}^{*} \Omega_{(0, q)}^{+-}$mod $\bar{\partial} r(z)$ converges uniformly in a neighborhood of $S$ to $\widetilde{\Omega}$. Here $\widetilde{\Omega}$ is

$$
\frac{\left(h\left(d \zeta_{*}^{\prime}, \overline{\zeta_{*}^{\prime}}\right)+A\left(d \zeta_{*}^{\prime}, \zeta_{*}^{\prime}\right)\right) \wedge\left(d \zeta_{*}^{n}+2 i A\left(d \zeta_{*}^{\prime}, \zeta_{*}^{\prime}\right)\right) \wedge h\left(d \zeta_{*}^{\prime}, d \overline{\zeta_{*}^{\prime}}\right)^{n-q-2} \wedge h\left(d \zeta_{*}^{\prime}, d \overline{z^{\prime}}\right)^{q}}{-(2 i)^{1-n} \bar{N}_{*}^{n-q-1} N_{*}^{q+1}\left(1-i \bar{N}_{*}^{-1} \zeta_{*}^{\prime} A \zeta_{*}^{\prime T}\right)^{n-q-1}\left(1+i N_{*}^{-1} \zeta_{*}^{\prime} A \zeta_{*}^{\prime T}\right)^{q+1}}
$$

Here $N_{*}=N_{*}\left(\zeta_{*}^{\prime}, \xi_{*}^{n}\right)$. Note that except at the origin, the above form is smooth in the $\left(\zeta_{*}^{\prime}, \xi_{*}^{n}\right)$-space. Therefore, for $\varphi(\zeta)=\sum_{|I|=q} \varphi_{\bar{I}}(\zeta) d \bar{\zeta}^{\prime} I$

$$
\begin{aligned}
\int_{2|F(\zeta, z)|=t^{2}} \varphi(\zeta) \wedge \Omega_{0, q}^{+-}(\zeta, z)=\int_{\left(\zeta_{*}^{\prime}, \xi_{*}^{n}\right) \in S_{t}(z)} \varphi\left(\zeta\left(\zeta_{*}^{\prime}, \xi_{*}^{n}\right)\right) \wedge \Omega_{0, q}^{+-}\left(\zeta\left(\zeta_{*}^{\prime}, \xi_{*}^{n}\right), z\right) \\
=\int_{\left(\zeta_{*}^{\prime}, \xi_{*}^{n}\right) \in T_{t}^{-1} S_{t}(z)} T_{t}^{*}\left\{\varphi\left(\zeta\left(\zeta_{*}^{\prime}, \xi_{*}^{n}\right)\right) \wedge \Omega_{0, q}^{+-}\left(\zeta\left(\zeta_{*}^{\prime}, \xi_{*}^{n}\right), z\right)\right\} \\
=\sum_{|I|=q} \varphi_{\bar{I}}(z) \int_{\left(\zeta_{*}^{\prime}, \xi_{*}^{n}\right) \in T_{t}^{-1} S_{t}(z)} T_{t}^{*}\left\{d \bar{\zeta}_{*}^{\prime}{ }^{I} \wedge \Omega_{0, q}^{+-}\left(\zeta\left(\zeta_{*}^{\prime}, \xi_{*}^{n}\right), z\right)\right\} \bmod \bar{\partial} r(z) .
\end{aligned}
$$

We obtain

$$
\lim _{t \rightarrow 0} \int_{2|F(\zeta, z)|=t^{2}} \varphi(\zeta) \wedge \Omega_{0, q}^{+-}(\zeta, z)=\sum_{|I|=q} \varphi_{\bar{I}}(z) \int_{S} d \bar{\zeta}_{*}^{I} \wedge \widetilde{\Omega}\left(\zeta_{*}^{\prime}, \xi_{*}^{n}, z\right) \bmod \bar{\partial} r(z)
$$

Return to $\widetilde{\Omega}$, defined by (B.19). We are ready to remove $A$ via an averaging. By (B.1), $|A|$ is small. Express $\left(1-i \bar{N}_{*}^{-1} \zeta_{*}^{\prime} A \zeta_{*}^{\prime T}\right)^{-(n-q-1)}\left(1+i N_{*}^{-1} \zeta_{*}^{\prime} A \zeta_{*}^{\prime T}\right)^{-(q+1)}$ as a convergent power series in $\bar{N}_{*}^{-1} \zeta_{*}^{\prime} A \zeta_{*}^{\prime T}$ and $N_{*}^{-1} \zeta_{*}^{\prime} A \zeta_{*}^{\prime T}$. Note that $N_{*}\left(\xi_{*}\right)$ is invariant under the rotation $e_{\theta}:\left(\zeta_{*}^{\prime}, \xi^{n}\right) \rightarrow\left(e^{i \theta} \zeta_{*}^{\prime}, \xi_{*}^{n}\right)$. Using $|I|=q$, we can verify that

$$
\begin{aligned}
\int_{\left(\zeta_{*}^{\prime}, \xi_{*}^{n}\right) \in S} d \bar{\zeta}_{*}^{I} \wedge \widetilde{\Omega}\left(\zeta_{*}^{\prime}, \xi_{*}^{n}, z\right) & =\frac{1}{2 \pi} \int_{0}^{2 \pi} d \theta \int_{\left(\zeta_{*}^{\prime}, \xi_{*}^{n}\right) \in e_{\theta} S} d \overline{\zeta_{*}^{\prime}} I \wedge \widetilde{\Omega}\left(\zeta_{*}^{\prime}, \xi_{*}^{n}, z\right) \\
& =\frac{1}{2 \pi} \int_{0}^{2 \pi} d \theta \int_{\left(\zeta_{*}^{\prime}, \xi_{*}^{n}\right) \in S} e_{\theta}^{*}\left\{d \overline{\zeta_{*}^{\prime}} I \wedge \widetilde{\Omega}\right\} \\
& =\frac{1}{2 \pi} \int_{\left(\zeta_{*}^{\prime}, \xi_{*}^{n}\right) \in S} \int_{\theta=0}^{2 \pi} e_{\theta}^{*}\left\{d \bar{\zeta}_{*}^{\prime} \wedge \widetilde{\Omega}\right\} d \theta .
\end{aligned}
$$

Therefore $\int_{\left(\zeta_{*}^{\prime}, \xi_{*}^{n}\right) \in S} d{\overline{\zeta_{*}^{\prime}}}^{I} \wedge \widetilde{\Omega}\left(\zeta_{*}^{\prime}, \xi_{*}^{n}, z\right)=\int_{\left(\zeta_{*}^{\prime}, \xi_{*}^{n}\right) \in S} d{\overline{\zeta_{*}^{\prime}}}^{I} \wedge \Omega^{\prime}\left(\zeta_{*}^{\prime}, \xi_{*}^{n}, z\right)$, where

$$
\Omega^{\prime}\left(\zeta_{*}^{\prime}, \xi_{*}^{n}, z\right)=\frac{d \zeta_{*}^{\prime} h{\overline{\zeta_{*}^{\prime}}}^{T} \wedge d \zeta_{*}^{n} \wedge\left(d \zeta_{*}^{\prime} \wedge h d{\overline{\zeta_{*}^{\prime}}}^{T}\right)^{n-q-2} \wedge\left(d \zeta_{*}^{\prime} \wedge h d{\overline{z^{\prime}}}^{T}\right)^{q}}{-(2 i)^{1-n} \overline{N_{*}^{n-q-1}\left(\xi_{*}\right)} N_{*}^{q+1}\left(\xi_{*}\right)}
$$


In eliminating $A$, we have used the fact that there are no terms $\bar{\zeta}_{*}^{\alpha} \bar{\zeta}_{*}^{\beta}$ in (B.16)(B.17).

Take a linear transformation $\hat{\zeta}^{\prime}=U\left(\zeta_{*}^{\prime}\right), \hat{\xi}^{n}=\xi_{*}^{n}$ such that

$$
h\left(\zeta_{*}^{\prime}, \overline{\zeta_{*}}\right)=\left|\hat{\zeta}^{\prime}\right|^{2}, \quad \hat{\zeta}^{n}=\hat{\xi}^{n}+i\left|\hat{\zeta}^{\prime}\right|^{2}
$$

Let $\hat{z}^{\prime}=U\left(z^{\prime}\right)$. Under the new coordinates and on $\left|\hat{\zeta}^{n}\right|=1, \Omega^{\prime}\left(\zeta_{*}^{\prime}, \xi_{*}^{n}, z\right)$ becomes

$$
\begin{aligned}
\widehat{\Omega}\left(\hat{\zeta}^{\prime}, \hat{\xi}^{n}, \hat{z}^{\prime}\right) & =\frac{\left(\overline{\hat{\zeta}^{\prime}} \cdot d \hat{\zeta}^{\prime}\right) \wedge d \hat{\zeta}^{n} \wedge\left(d \hat{\zeta}^{\prime} \dot{\wedge} d \overline{\hat{\zeta}^{\prime}}\right)^{n-q-2} \wedge\left(d \hat{\zeta}^{\prime} \dot{\wedge} d \overline{\hat{z}^{\prime}}\right)^{q}}{-(2 i)^{1-n}\left(\overline{\hat{\zeta}^{n}}\right)^{n-q-1}\left(\hat{\zeta}^{n}\right)^{q+1}} \\
& =\left(\overline{\hat{\zeta}^{\prime}} \cdot d \hat{\zeta}^{\prime}\right) \wedge d \mu\left(\hat{\zeta}^{n}\right) \wedge\left(d \hat{\zeta}^{\prime} \dot{\wedge} d \overline{\hat{\zeta}^{\prime}}\right)^{n-q-2} \wedge\left(d \hat{\zeta}^{\prime} \dot{\wedge} d \overline{\hat{z}^{\prime}}\right)^{q}
\end{aligned}
$$

Here

$$
\mu\left(\hat{\zeta}^{n}\right)= \begin{cases}-(2 i)^{n-1}(n-2 q-1)^{-1}\left(\hat{\zeta}^{n}\right)^{n-2 q-1}, & n-2 q \neq 1 \\ -(2 i)^{n-1} \log \hat{\zeta}^{n}, & n-2 q=1\end{cases}
$$

Note that $\operatorname{Im} \hat{\zeta}^{n} \geq 0$ and hence $\mu\left(\hat{\zeta}^{n}\right)$ is smooth and single-valued on $\left|\hat{\zeta}^{n}\right|=1$. To remove the differential on $\mu$, we apply Stokes' theorem and get

$$
\begin{aligned}
c_{q} d{\overline{\hat{z}^{\prime}}}^{I} & \stackrel{\text { def }}{=} \int_{\left|\hat{\zeta}^{n}\right|=1} d \overline{\hat{\zeta}}^{I} \wedge\left(\overline{\hat{\zeta}}^{\prime} \cdot d \hat{\zeta}^{\prime}\right) \wedge d \mu\left(\hat{\zeta}^{n}\right) \wedge\left(d \hat{\zeta}^{\prime} \dot{\wedge} d \overline{\hat{\zeta}^{\prime}}\right)^{n-q-2} \wedge\left(d \hat{\zeta}^{\prime} \dot{\wedge} d \overline{\hat{z}^{\prime}}\right)^{q} \\
& =-\int_{\left|\hat{\zeta}^{n}\right|=1} \mu\left(\hat{\zeta}^{n}\right)\left(d \hat{\zeta}^{\prime} \dot{\wedge} d \overline{\hat{\zeta}}^{\prime}\right)^{n-q-1} \wedge d \overline{\hat{\zeta}}^{I} \wedge\left(d \hat{\zeta}^{\prime} \dot{\wedge} d \overline{\hat{z}}^{\prime}\right)^{q} .
\end{aligned}
$$

Now, the differential form is a multiple of the volume-form on $\mathbb{C}^{n-1}$ (the $\hat{\zeta}^{\prime}$-space). The projection from $\left|\hat{\zeta}^{n}\right|=1$ to the ball $\left|\hat{\zeta}^{\prime}\right| \leq 1$ is two-to-one, branched over $\left|\hat{\zeta}^{\prime}\right|=1$. Recall that $d V(\zeta)=d \xi^{1} \wedge d \eta^{1} \wedge \cdots \wedge d \eta^{n-1} \wedge d \xi^{n}$ defines the orientation of $M$ and $\left(\zeta^{\prime}, \xi^{n}\right) \rightarrow\left(\zeta_{*}^{\prime}, \xi_{*}^{n}\right)$ preserves the orientation. Since $S:\left|\hat{\zeta}^{n}\right|=1$ is obtained via the dilation of the boundary of $|F(z, \cdot)|<t, S$ must be oriented as the boundary of $\left|\hat{\zeta}^{n}\right|<1$ on which $d V(\hat{\zeta})$ is the volume-form. After considering the orientation, we conclude that

$$
c_{q} d{\overline{\hat{z}^{\prime}}}^{I}=\int_{\left|\hat{\zeta}^{\prime}\right| \leq 1}\left(\mu\left(\hat{\zeta}_{-}^{n}\right)-\mu\left(\hat{\zeta}_{+}^{n}\right)\right)\left(d \hat { \zeta } ^ { \prime } \dot { \wedge } d { \overline { \hat { \zeta } ^ { \prime } } } ^ { n - q - 1 } \wedge d { \overline { \hat { \zeta } ^ { \prime } } } ^ { I } \wedge \left(d \hat{\zeta}^{\prime} \dot{\wedge} d{\hat{z^{\prime}}}^{q}\right.\right.
$$

Here $d V\left(\hat{\zeta}^{\prime}\right)=d \hat{\xi}^{1} \wedge d \hat{\eta}^{1} \wedge \cdots \wedge d \hat{\eta}^{n-1}$ is the volume-form on the $\hat{\zeta}^{\prime}$-space, and

$$
\hat{\zeta}_{+}^{n}=\left(1-\left|\hat{\zeta}^{\prime}\right|^{4}\right)^{1 / 2}+i\left|\hat{\zeta}^{\prime}\right|^{2}, \quad \hat{\zeta}_{-}^{n}=-\left(1-\left|\hat{\zeta}^{\prime}\right|^{4}\right)^{1 / 2}+i\left|\hat{\zeta}^{\prime}\right|^{2} .
$$

To rewrite the integrand, introduce variables $\xi=\left(\xi_{1}, \cdots, \xi_{q}\right), \eta, x, y$ so that

$$
d{\overline{\hat{\zeta}^{\prime}}}^{I}=d \bar{\xi}^{I}, \quad d \hat{\zeta}^{\prime} \dot{\wedge} d \overline{\hat{\zeta}^{\prime}}=d \xi \dot{\wedge} d \bar{\xi}+d \eta \dot{\wedge} d \bar{\eta}, \quad d \hat{\zeta}^{\prime} \dot{\wedge} d \overline{\hat{z}^{\prime}}=d \xi \dot{\wedge} d \bar{x}+d \eta \dot{\wedge} d \bar{y} .
$$


Then $(d \xi \dot{\wedge} d \bar{\xi}+d \eta \dot{\wedge} d \bar{\eta})^{n-q-1} \wedge d \bar{\xi}^{I} \wedge(d \xi \dot{\wedge} d \bar{x}+d \eta \dot{\wedge} d \bar{y})^{q}$ equals

$$
\begin{aligned}
(d \eta \dot{\wedge} d \bar{\eta})^{n-q-1} & \wedge d \bar{\xi}^{I} \wedge(d \xi \dot{\wedge} d \bar{x}+d \eta \dot{\wedge} d \bar{y})^{q} \\
& =(d \eta \dot{\wedge} d \bar{\eta})^{n-q-1} \wedge d \bar{\xi}^{I} \wedge(d \xi \dot{\wedge} d \bar{x})^{q} \\
& =(-1)^{q}(d \eta \dot{\wedge} d \bar{\eta})^{n-q-1} \wedge(d \xi \dot{\wedge} d \bar{\xi})^{q} \wedge d \bar{x}^{I}
\end{aligned}
$$

The last term equals $(-1)^{q}\left(\begin{array}{c}n-1 \\ q\end{array}\right)^{-1}(d \xi \dot{\wedge} d \bar{\xi}+d \eta \dot{\wedge} d \bar{\eta})^{n-1} \wedge d \bar{x}^{I}$. Therefore

$$
c_{q} d{\overline{\hat{z}^{\prime}}}^{I}=(-1)^{q}\left(\begin{array}{c}
n-1 \\
q
\end{array}\right)^{-1} \int_{\left|\hat{\zeta}^{\prime}\right|<1}\left(\mu\left(\hat{\zeta}_{-}^{n}\right)-\mu\left(\hat{\zeta}_{+}^{n}\right)\right)\left(d \hat{\zeta}^{\prime} \dot{\wedge} d{\overline{\hat{\zeta}^{\prime}}}^{n-1} \wedge d{\overline{\bar{z}^{\prime}}}^{I} .\right.
$$

We now compute $c_{q}$. Using the polar coordinates, we get

$$
\begin{aligned}
c_{q} & =(-1)^{q}\left(\begin{array}{c}
n-1 \\
q
\end{array}\right)^{-1}(n-1) !(-2 i)^{n-1} \int_{\left|\hat{\zeta}^{\prime}\right|<1}\left(\mu\left(\hat{\zeta}_{-}^{n}\right)-\mu\left(\hat{\zeta}_{+}^{n}\right)\right) d V\left(\hat{\xi}^{\prime}\right) \\
& =\tilde{c}_{q} \int_{0}^{1}\left(\mu\left(\left(1-r^{4}\right)^{1 / 2}+i r^{2}\right)-\mu\left(-\left(1-r^{4}\right)^{1 / 2}+i r^{2}\right)\right) r^{2 n-3} d r \\
& =\frac{\tilde{c}_{q}}{2(n-1)} \int_{r=0}^{1} r^{2 n-2} d\left\{\mu\left(\left(1-r^{4}\right)^{1 / 2}+i r^{2}\right)-\mu\left(-\left(1-r^{4}\right)^{1 / 2}+i r^{2}\right)\right\} .
\end{aligned}
$$

Note that the last identity is obtained by integration by parts. Here

$$
\tilde{c}_{q}=(-1)^{q+1}\left(\begin{array}{c}
n-1 \\
q
\end{array}\right)^{-1}(n-1) !(-2 i)^{n-1} \sigma_{n-1}, \quad \sigma_{n-1}=\frac{2 \pi^{n-1}}{(n-2) !} .
$$

Letting $r^{2}=s$ and $z=\left(1-s^{2}\right)^{1 / 2}+i s$, we get

$$
\begin{aligned}
c_{q} & =\frac{\tilde{c}_{q}}{2(n-1)} \int_{s=-1}^{1} s^{n-1} d\left\{\mu\left(\left(1-s^{2}\right)^{1 / 2}+i s\right)\right\} \\
& =\frac{\tilde{c}_{q}}{4(n-1)} \int_{|z|=1}(\operatorname{Im} z)^{n-1} d \mu(z)=\frac{-(2 i)^{n-1} \tilde{c}_{q}}{4(n-1)} \int_{|z|=1}(\operatorname{Im} z)^{n-1} z^{n-2 q-2} d z .
\end{aligned}
$$

A simple residue computation yields $c_{q}=\frac{1}{2}(2 \pi i)^{n}$ for $0 \leq q \leq n-1$. The proof of Lemma B.3 is complete.

We need the following lemma from [7], where $|F(\zeta, z)|=t$ is replaced by $|\zeta-z|=t$ and only $r \in \mathcal{C}^{2}$ is needed.

Lemma B.4. Let $1 \leq q \leq n-1$. Let $M, \rho_{0}$ be as in Lemma B.3. Assume that $r$ is of class $\mathcal{C}^{3}$. Let $\varphi$ be a continuous $(0, q)$-form on $M_{\rho_{0}}$. If $0<\rho<\rho_{0}$ and $t>0$ is sufficiently small, then on $M_{\rho}$, in the sense of currents and modulo $\bar{\partial} r(z)$,

$\int_{F(\zeta, z)>t} \varphi(\zeta) \wedge \bar{\partial}_{z} \Omega_{(0, q-1)}^{+-}(\zeta, z)=(-1)^{q-1} \bar{\partial}_{b} \int_{F(\zeta, z)>t} \varphi(\zeta) \wedge \Omega_{(0, q-1)}^{+-}(\zeta, z)$. 
Proof. For the convenience of the reader, we reproduce the proof in [7]. Since $r \in \mathcal{C}^{3}$, for $t$ sufficiently small, both integrals are continuous on $M_{\rho}$. To verify the identity in the sense of currents, let $\psi$ be a smooth $(n, n-1-q)$-form with compact support in $M_{\rho}$. Interchanging the order of integration, we have

$$
\begin{aligned}
\mathcal{I}(z)=\int_{z \in M} & \int_{F(\zeta, z)>t} \varphi(\zeta) \wedge \bar{\partial}_{z} \Omega_{(0, q-1)}^{+-}(\zeta, z) \wedge \psi(z) \\
& =-\int_{\zeta \in M} \int_{F(\zeta, z)>t} \varphi(\zeta) \wedge \bar{\partial}_{z} \Omega_{(0, q-1)}^{+-}(\zeta, z) \wedge \psi(z) \quad \text { (by Fubini) } \\
& =\int_{\zeta \in M} \int_{F(\zeta, z)>t} \psi(z) \wedge \bar{\partial}_{z} \Omega_{(0, q-1)}^{+-}(\zeta, z) \wedge \varphi(\zeta) .
\end{aligned}
$$

Applying Stokes' theorem yields

$$
\begin{aligned}
\mathcal{I}(z)= & (-1)^{q} \int_{\zeta \in M} \int_{F(\zeta, z)>t} \bar{\partial}_{z} \psi(z) \wedge \Omega_{(0, q-1)}^{+-}(\zeta, z) \wedge \varphi(\zeta) \\
& +(-1)^{q+1} \int_{\zeta \in M} \int_{F(\zeta, z)=t} \psi(z) \wedge \Omega_{(0, q-1)}^{+-}(\zeta, z) \wedge \varphi(\zeta) \\
= & \int_{\zeta \in M} \int_{F(\zeta, z)>t} \varphi(\zeta) \wedge \Omega_{(0, q-1)}^{+-}(\zeta, z) \wedge \bar{\partial}_{z} \psi(z) \\
& +(-1)^{q+1} \int_{\zeta \in M} \int_{F(\zeta, z)=t} \varphi(\zeta) \wedge \Omega_{(0, q-1)}^{+-}(\zeta, z) \wedge \psi(z) .
\end{aligned}
$$

Interchanging the order of integration in both terms yields

$$
\begin{aligned}
\mathcal{I}(z)= & -\int_{z \in M} \int_{F(\zeta, z)>t} \varphi(\zeta) \wedge \Omega_{(0, q-1)}^{+-}(\zeta, z) \wedge \bar{\partial}_{z} \psi(z) \\
& +(-1)^{q+1} \int_{z \in M} \int_{F(\zeta, z)=t} \varphi(\zeta) \wedge \Omega_{(0, q-1)}^{+-}(\zeta, z) \wedge \psi(z) \\
= & -\int_{z \in M} \int_{F(\zeta, z)>t} \varphi(\zeta) \wedge \Omega_{(0, q-1)}^{+-}(\zeta, z) \wedge \bar{\partial}_{z} \psi(z) .
\end{aligned}
$$

Here the second last term vanishes by counting total degree in $\zeta$, which equals $q+2 n-q-1>2 n-2$.

As in [21], the above two lemmas can be used to derive the Henkin homotopy formula for $(0, q)$-forms on $\bar{M}_{\rho}$ via Stokes' theorem, when $r \in \mathcal{C}^{3}$ satisfies (B.1) and $\varphi \in \mathcal{C}^{1}\left(\overline{M_{\rho_{0}}}\right)$. Here are the details. Let $M^{t}(z)=M_{\rho_{0}} \cap\{\zeta:|F(\zeta, z)|>t\}$. We will use $\bar{\partial}_{\zeta} \Omega_{(0, q)}^{+-}+\bar{\partial}_{z} \Omega_{(0, q)}^{+-}=0$ for $\zeta \neq z$ and $1 \leq q \leq n-2$; see [21]. For 
$z \in M_{\rho}$ and $t$ sufficiently small,

$$
\begin{aligned}
& \int_{\partial M^{t}(z)} \varphi(\zeta) \wedge \Omega_{(0, q)}^{+-}(\zeta, z) \\
& \quad=\int_{M^{t}(z)} \bar{\partial}_{\zeta} \varphi \wedge \Omega_{(0, q)}^{+-}+(-1)^{q} \int_{M^{t}(z)} \varphi(\zeta) \wedge \bar{\partial}_{\zeta} \Omega_{(0, q)}^{+-} \\
& \quad=\int_{M^{t}(z)} \bar{\partial}_{\zeta} \varphi \wedge \Omega_{(0, q)}^{+-}(\zeta, z)+(-1)^{q-1} \int_{M^{t}(z)} \varphi(\zeta) \wedge \bar{\partial}_{z} \Omega_{(0, q-1)}^{+-}(\zeta, z)
\end{aligned}
$$

Using Lemmas B.3-B.4 and letting $t \rightarrow 0$, we obtain, modulo $\bar{\partial} r(z)$,

$$
\int_{\partial M_{\rho_{0}}} \varphi(\zeta) \wedge \Omega_{(0, q)}^{+-}(\zeta, z)=c_{0} \varphi(z)+\int_{M_{\rho_{0}}} \bar{\partial}_{\zeta} \varphi \wedge \Omega_{(0, q)}^{+-}+\bar{\partial}_{b} \int_{M_{\rho_{0}}} \varphi(\zeta) \wedge \Omega_{(0, q-1)}^{+-}
$$

Now assume that $0<q<n-2$. Then $-\Omega_{(0, q)}^{+-}=\bar{\partial}_{\zeta} \Omega_{(0, q)}^{0+-}+\bar{\partial}_{z} \Omega_{(0, q-1)}^{0+-}$; see [21].

We get that modulo $\bar{\partial} r(z)$

$$
\begin{aligned}
& \int_{\partial M_{\rho_{0}}} \varphi(\zeta) \wedge \Omega_{(0, q)}^{+-}(\zeta, z) \\
& \quad=-\int_{\partial M_{\rho_{0}}} \varphi(\zeta) \wedge \bar{\partial}_{\zeta} \Omega_{(0, q)}^{0+-}-\int_{\partial M_{\rho_{0}}} \varphi(\zeta) \wedge \bar{\partial}_{z} \Omega_{(0, q-1)}^{0+-} \\
& \quad=(-1)^{q} \int_{\partial M_{\rho_{0}}} \bar{\partial}_{b} \varphi(\zeta) \wedge \Omega_{(0, q)}^{0+-}-(-1)^{q} \bar{\partial}_{b} \int_{\partial M_{\rho_{0}}} \varphi(\zeta) \wedge \Omega_{(0, q-1)}^{0+-}
\end{aligned}
$$

Therefore, modulo $\bar{\partial} r(z)$ and as currents,

$$
\begin{aligned}
c_{0} \varphi(z)= & \bar{\partial}_{b}\left\{-\int_{M_{\rho_{0}}} \varphi(\zeta) \wedge \Omega_{(0, q-1)}^{+-}(\zeta, z)-(-1)^{q} \int_{\partial M_{\rho_{0}}} \varphi(\zeta) \wedge \Omega_{(0, q-1)}^{0+-}(\zeta, z)\right\} \\
& +\left\{-\int_{M_{\rho_{0}}} \bar{\partial}_{b} \varphi(\zeta) \wedge \Omega_{(0, q)}^{+-}(\zeta, z)+(-1)^{q} \int_{\partial M_{\rho_{0}}} \bar{\partial}_{b} \varphi(\zeta) \wedge \Omega_{(0, q)}^{0+-}(\zeta, z)\right\} \\
= & -\bar{\partial}_{b}\left\{\int_{M_{\rho_{0}}} \Omega_{(0, q-1)}^{+-}(\zeta, z) \wedge \varphi(\zeta)+\int_{\partial M_{\rho_{0}}} \Omega_{(0, q-1)}^{0+-}(\zeta, z) \wedge \varphi(\zeta)\right\} \\
& -\left\{\int_{M_{\rho_{0}}} \Omega_{(0, q)}^{+-}(\zeta, z) \wedge \bar{\partial}_{b} \varphi(\zeta)+\int_{\partial M_{\rho_{0}}} \Omega_{(0, q)}^{0+-}(\zeta, z) \wedge \bar{\partial}_{b} \varphi(\zeta)\right\} .
\end{aligned}
$$


We now derive the homotopy formula by reducing the regularity condition. Notice that when $M$ is $\mathcal{C}^{2}$ and strictly convex it is proved by Henkin [21].

Fix a tangential $(0, q)$-form $\varphi$ on $M_{\rho_{0}}$. Let $0<q<n-2$.

Case a) $r \in \mathcal{C}^{2}$ and $\varphi \in \mathcal{C}^{1}\left(\bar{M}_{\rho_{0}}\right)$. Write $x=\left(\operatorname{Re} z, \operatorname{Im} z^{\prime}\right)$ and $\xi=\left(\operatorname{Re} \zeta, \operatorname{Im} \zeta^{\prime}\right)$ with $\zeta, z \in M_{\rho_{0}}$. We first consider the case when $\varphi$ has compact support, say in $M_{\rho_{1}}$ with $\rho_{1}<\rho_{0}$. Then $P_{M_{\rho_{0}}}^{\prime} \varphi$ has no boundary term. Recall that $\Psi(\xi, x)=$ $\left(\tilde{\psi}_{x}(\xi), x\right)=\left(\xi_{*}, x\right)$ is defined by relations $\zeta_{*}^{\prime}=\zeta^{\prime}-z^{\prime}, \zeta_{*}^{n}=-2 i r_{z}(\zeta-z)$ and $\zeta, z \in M_{\rho_{0}}$. By (8.2)-(8.3) and (8.6), on $D_{\rho_{0}}$

$$
\begin{gathered}
P_{0, M_{\rho_{0}}}^{\prime} \varphi(x)=\sum_{|I|=q-1} \mathcal{I}_{\bar{I}}(x) d{\overline{z^{\prime}}}^{I}, \\
\mathcal{I}_{\bar{I}}(x)=\sum_{|K|=1} \sum_{|J|=q} \int_{B_{9 \rho_{0}}}\left(\left(\varphi_{\bar{J}} \tilde{A}_{\bar{I} K}^{\bar{J}}\right) \circ \Psi^{-1} \cdot \hat{T}_{1}^{-a} \cdot \hat{T}_{2}^{-b}\right)\left(\xi_{*}, x\right) \cdot \hat{k}_{a b}^{K}\left(\xi_{*}\right) d V\left(\xi_{*}\right), \\
\varphi_{\bar{J}}(\xi, x)=\varphi_{\bar{J}}(\xi), \quad \hat{T}_{1}\left(\xi_{*}, x\right)=1+\sum_{|L|=2} C_{L} \circ \Psi^{-1}\left(\xi_{*}, x\right) \xi_{*}^{L} N_{*}^{-1}\left(\xi_{*}\right) .
\end{gathered}
$$

Here $\tilde{A}_{\bar{I} K}^{\bar{J}}, C_{L}$ are functions of the form $\partial_{*}^{2} r, \partial_{*}^{2} \hat{r}$, respectively, defined in Section 8 . An analogous formula holds for $\hat{T}_{2}$. These functions depend only on derivatives of $\hat{r}$ of order at most two. We take a sequence of $\mathcal{C}^{\infty}$ functions $\hat{r}^{m}$ converging to $\hat{r}$ in $\mathcal{C}^{2}$-norm on $\bar{D}_{\rho_{0}}$. Subtracting $\hat{r}^{m}$ by its Taylor polynomial of order 1 about the origin, we may assume that $\hat{r}^{m}(0)=0$ and $\partial \hat{r}^{m}(0)=0$. In what follows we use the letter $m$ to indicate dependence on $\hat{r}^{m}$. Let $M^{m}$ be the graph $y^{n}=\left|z^{\prime}\right|^{2}+\hat{r}^{m}(x)$ over $D_{\rho_{0}}$. Let $\rho \in\left(\rho_{1}, \rho_{0}\right)$. There exists $m_{*}$ such that for $m>m_{*}$

$$
D_{\rho_{1}} \subset \bar{D}_{\rho}^{m} \subset D_{\rho_{0}}, \quad D_{\rho}^{m} \stackrel{\text { def }}{=}\left\{x \in D_{\rho_{0}}:|x|^{2}+\hat{r}^{m}(x)<\rho^{2}\right\} .
$$

Assume that $m>m_{*}$. Then by $\left\|\hat{r}^{m}\right\|_{D_{\rho_{0}}, 2}<1 / C_{0}, D_{\rho}^{m}$ is strictly convex. Note that $\varphi$ is still a tangential $(0, q)$-form of $M^{m}$ on $D_{\rho_{0}}$. By the homotopy formula for the smooth $M^{m}$,

$$
\varphi=\bar{\partial}_{M^{m}} P_{M_{\rho}^{m}}^{\prime} \varphi+Q_{M_{\rho}^{m}}^{\prime} \bar{\partial}_{M^{m}} \varphi .
$$

By the formula of $\hat{T}_{j}$, it is clear that $\left|\hat{T}_{j, m}\right| \geq 1 / 2$ for $m$ sufficiently large and $0 \neq \xi_{*} \in B_{9 \rho} \cap \tilde{\psi}_{x}^{m}\left(D_{\rho}\right)$. Since $\varphi_{\bar{J}}$ has compact support, we have

$$
\left|\left\{\left(\varphi_{\bar{J}} \tilde{A}_{\bar{I} K}^{\bar{J} m}\right) \circ \Psi_{m}^{-1} \cdot \hat{T}_{1, m}^{-a} \cdot \hat{T}_{2, m}^{-b}\right\}\left(\xi_{*}, x\right)\right| \leq C, \quad \xi_{*} \neq 0 .
$$

Since $\hat{k}_{a b}^{I} \in L_{\text {loc }}^{1}$, by the dominated convergence theorem, $\mathcal{I}_{\bar{I}}^{m}$ converges to $\mathcal{I}_{\bar{I}}$ pointwise on $\bar{D}_{\rho_{1}}$ as $m \rightarrow \infty$. Thus, $P_{M_{\rho}^{m}}^{\prime} \varphi$ converges to $P_{M_{\rho}}^{\prime} \varphi$ pointwise on $\bar{D}_{\rho_{1}}$. 
By the $\mathcal{C}^{0}$-estimate on $P_{0}^{\prime}$ (see (9.3)), we know that $\left|P_{M_{\rho}^{m}}^{\prime} \varphi\right|<C$ on $\bar{D}_{\rho_{1}} \subset \cap_{m} D_{\rho}^{m}$. Since $\varphi$ is of class $\mathcal{C}^{1}, \bar{\partial}_{M^{m}} \varphi$ converges to $\bar{\partial}_{M} \varphi$ uniformly on $\bar{D}_{\rho_{0}}$. Next, we fix an $(n, n-q-1)$-form $\psi=\sum_{|J|=n-q-1} \psi_{\bar{J}} d z^{1} \wedge \cdots \wedge d z^{n-1} \wedge \partial r(z) \wedge d{\overline{z^{\prime}}}^{J}$ of class $\mathcal{C}^{1}$ and of compact support in $D_{\rho_{1}}$. Then $\psi^{m}=\sum_{|J|=n-q-1} \psi_{\bar{J}} d z^{1} \wedge$ $\cdots \wedge d z^{n-1} \wedge \partial r^{m}(z) \wedge d{\overline{z^{\prime}}}^{J}$ is an $(n, n-1-q)$-form of $M^{m}$ on $D_{\rho}^{m}$. Obviously, $\psi^{m}$ converges to $\psi$ in $\mathcal{C}^{1}$-norm uniformly on $\mathbb{R}^{2 n-1}$. Now one can see that $\int_{D_{\rho_{1}}} P_{0, M_{\rho}^{m}}^{\prime} \varphi \wedge \bar{\partial}_{M^{m}} \psi^{m}$ converges to $\int_{D_{\rho_{1}}} P_{0, M_{\rho}}^{\prime} \varphi \wedge \bar{\partial}_{M} \psi$. A similar argument shows that $Q_{0, M_{\rho}^{m}}^{\prime} \bar{\partial}_{M^{m}} \varphi$ are uniformly bounded and converge to $Q_{0, M_{\rho}}^{\prime} \bar{\partial}_{M} \varphi$ pointwise on $D_{\rho_{1}}$. Thus $\int_{D_{\rho_{1}}}\left(Q_{0, M_{\rho}^{m}}^{\prime} \bar{\partial}_{M^{m}} \varphi\right) \wedge \psi^{m}$ converges to $\int_{D_{\rho_{1}}}\left(Q_{0, M_{\rho}}^{\prime} \bar{\partial}_{M} \varphi\right) \wedge \psi$. By the homotopy formula (B.20),

$$
\begin{aligned}
\int_{D_{\rho_{1}}} \varphi \wedge \psi^{m}= & (-1)^{q} \int_{D_{\rho_{1}}}\left(P_{0, M_{\rho}^{m}}^{\prime} \varphi\right) \wedge \bar{\partial}_{M^{m}} \psi^{m} \\
& +\int_{D_{\rho_{1}}}\left(Q_{0, M_{\rho}^{m}}^{\prime} \bar{\partial}_{M^{m}} \varphi\right) \wedge \psi^{m} .
\end{aligned}
$$

Taking limits, we see that as currents, $\varphi=\bar{\partial}_{M} P_{0, M_{\rho}}^{\prime} \varphi+Q_{0, M_{\rho}}^{\prime} \bar{\partial}_{M} \varphi$ holds on $D_{\rho_{1}}$. Since $\varphi$ has compact support in $D_{\rho_{1}} \subset D_{\rho}$, we replace the domain of integration $M_{\rho}$ by $M_{\rho_{0}}$ and add boundary integrals. We get $\varphi=\bar{\partial}_{M} P_{M_{\rho_{0}}}^{\prime} \varphi+Q_{M_{\rho_{0}}}^{\prime} \bar{\partial}_{M} \varphi$ on $D_{\rho_{1}}$ as currents, and hence on $D_{\rho_{0}}$ whenever $\varphi$ has compact support in $D_{\rho_{0}}$.

Return to the general case. Let $M^{m}$ be as before. Take any $\rho_{1}, \rho_{2}, \rho$ such that $0<\rho_{1}<\rho_{2}<\rho<\rho_{0}$. Take a $\mathcal{C}^{\infty}$ function $\chi$ which is 1 on $D_{\rho_{2}}$ and has compact support in $D_{\rho_{0}}$. Let $\varphi_{0}=\chi \varphi$ and $\varphi_{1}=(1-\chi) \varphi$. We have proved that $\varphi_{0}=\bar{\partial}_{M} P_{M_{\rho_{0}}}^{\prime} \varphi_{0}+Q_{M_{\rho_{0}}}^{\prime} \bar{\partial}_{M} \varphi_{0}$ as currents on $D_{\rho_{0}}$. Let $\psi, \psi^{m}$ be as before, which are supported in $D_{\rho_{1}}$. By an analogy of (B.21), for $m>m_{*}$ we have

$$
\int_{D_{\rho_{1}}} \varphi_{1} \wedge \psi^{m}=(-1)^{q} \int_{D_{\rho_{1}}}\left(P_{M_{\rho}^{m}}^{\prime} \varphi_{1}\right) \wedge \bar{\partial}_{M^{m}} \psi^{m}+\int_{D_{\rho_{1}}}\left(Q_{M_{\rho}^{m}}^{\prime} \bar{\partial}_{M^{m}} \varphi_{1}\right) \wedge \psi^{m} .
$$

Since supp $\psi^{m} \subset D_{\rho_{1}}$ and supp $\varphi_{1} \subset \bar{D}_{\rho_{0}} \backslash D_{\rho_{2}}$, the dominated convergence theorem implies the convergence of $\int_{D_{\rho_{1}}}\left(P_{0, M_{\rho}^{m}}^{\prime} \varphi_{1}\right) \wedge \bar{\partial}_{M^{m}} \psi^{m}$ to $\int_{D_{\rho_{1}}}\left(P_{0, M_{\rho}}^{\prime} \varphi_{1}\right) \wedge \bar{\partial}_{M} \psi$. The integrands for the boundary integrals $\left(P_{1, M_{\rho}^{m}}^{\prime} \varphi_{1}\right)(x)$ are of class $\mathcal{C}^{1}$ in a neighborhood of $\partial D_{\rho}$, when $x \in D_{\rho_{2}}$. Note that the map $d_{\rho}$ sending $x \in \partial D_{\rho}$ to $r_{\rho}(x) x \in \partial D_{\rho}^{m}$ with $r_{\rho}>0$ converges to the identity map in the $\mathcal{C}^{2}$ norm as $m \rightarrow \infty$. Thus we can verify that $P_{1, M_{\rho}^{m}}^{\prime} \varphi_{1}$ converges uniformly to $P_{1, M_{\rho}}^{\prime} \varphi_{1}$ on $D_{\rho_{1}}$. Combining with supp $\psi^{m} \subset D_{\rho_{1}}$, we obtain the convergence of $\int_{D_{\rho_{1}}}\left(P_{1, M_{\rho}^{m}}^{\prime} \varphi_{1}\right) \wedge$ $\bar{\partial}_{M^{m}} \psi^{m}$ to $\int_{D_{\rho_{1}}}\left(P_{1, M_{\rho}}^{\prime} \varphi_{1}\right) \wedge \bar{\partial}_{M} \psi$. One can verify that $\int_{D_{\rho_{1}}}\left(Q_{1, M_{\rho}^{m}}^{\prime} \bar{\partial}_{M^{m}} \varphi_{1}\right) \wedge \psi^{m}$ converges to $\int_{D_{\rho_{1}}}\left(Q_{1, M_{\rho}}^{\prime} \bar{\partial}_{M} \varphi_{1}\right) \wedge \psi$. Hence $\varphi_{1}=\bar{\partial}_{M} P_{M_{\rho}} \varphi_{1}+Q_{M_{\rho}} \bar{\partial}_{M} \varphi_{1}$ holds 
as currents on $D_{\rho_{1}}$. The definition of $\varphi_{1}$ is independent of $\rho>\rho_{2}$. Letting $\rho \rightarrow \rho_{0}$, we get $\varphi_{1}=\bar{\partial}_{M} P_{M_{\rho_{0}}} \varphi_{1}+Q_{M_{\rho_{0}}} \bar{\partial}_{M} \varphi_{1}$ on $D_{\rho_{1}}$. Add to $\varphi_{0}$. We get $\varphi=\bar{\partial}_{M} P_{M_{\rho_{0}}} \varphi+Q_{M_{\rho_{0}}} \bar{\partial}_{M} \varphi$ as currents on $D_{\rho_{1}}$, and hence on $D_{\rho_{0}}$.

Case b) $r \in \mathcal{C}^{2}(D)$ and $\varphi, \bar{\partial}_{M} \varphi \in \mathcal{C}^{0}\left(\bar{M}_{\rho_{0}}\right)$. We verify the homotopy formula by the Friedrichs approximation theorem, for which we need the commutator of a smoothing operator $S_{t}$ and $\bar{\partial}_{M}$, applied to tangential $(0, q)$-forms.

Recall that on $M$

$$
\theta(\xi)=-2 i \partial r(\zeta)=a d \xi^{n} \quad \bmod \left(d \zeta^{\alpha}, d \bar{\zeta}^{\beta}\right), \quad a=1+\hat{r}_{\xi^{n}}^{2}
$$

Let $\varphi=\sum_{|I|=q} \varphi_{\bar{I}}(x) d{\overline{z^{\prime}}}^{I}$ be a continuous tangential $(0, q)$ form on $M$. Let $\chi$ be a smooth function of compact support in $\mathbb{R}^{2 n-1}$ such that $\int \chi d V=1$. Let $\chi_{t}(x)=$ $t^{1-2 n} \chi\left(t^{-1} x\right)$ and define $S_{t} \varphi=\sum_{|I|=q} \varphi_{\bar{I}} * \chi_{t} d{\overline{z^{\prime}}}^{I}$. Recall that $\bar{X}_{\alpha}=\partial_{\overline{z^{\alpha}}}+b_{\bar{\alpha}} \partial_{x^{n}}$ with $b_{\bar{\alpha}}=-r_{z^{\alpha}} /\left(2 r_{z^{n}}\right)$.

Lemma B.5. Let $0 \leq q<n-1$. Let $M \subset \mathbb{C}^{n}$ be a graph of class $\mathcal{C}^{2}$ over $D \subset \mathbb{C}^{n-1} \times \mathbb{R}$. Assume that $\varphi$ is a continuous tangential $(0, q)$-form on $M_{\rho}$ such that $\bar{\partial}_{M} \varphi$ is continuous on $M_{\rho}$. Let $0<\rho^{\prime}<\rho$. Then for $t$ sufficiently small and on $M_{\rho^{\prime}}$,

$$
\begin{aligned}
{\left[\bar{\partial}_{M}, S_{t}\right] \varphi(x)=\sum_{|I|=q, 1 \leq \alpha<n} \int } & \left\{\varphi_{\bar{I}}(x-t \xi) t^{-1}\left(b_{\bar{\alpha}}(x)-b_{\bar{\alpha}}(x-t \xi)\right)\left(\partial_{\xi^{n}} \chi\right)(\xi)\right. \\
& \left.-\left(\varphi_{\bar{I}} a^{-1} \bar{X}_{\alpha} a\right)(x-t \xi) \chi(\xi)\right\} d V(\xi) \wedge d \overline{z^{\alpha}} \wedge d{\overline{z^{\prime}}}^{I} .
\end{aligned}
$$

Proof. We follow a computation in [4]; see also [10, page 121]. Let $\varphi=\sum_{|I|=q} \varphi_{\bar{I}} d \overline{z^{\prime}}$.

By the assumption, we have $\bar{\partial}_{M} \varphi=\sum_{|J|=q+1} \psi_{\bar{J}} d{\overline{z^{\prime}}}^{J}$ with $\psi_{\bar{J}} \in \mathcal{C}^{0}$. Now

$$
\begin{aligned}
\bar{\partial}_{M} S_{t} \varphi(x) & =\sum_{|I|=q} \sum_{1 \leq \alpha<n} \int \varphi_{\bar{I}}(\xi) \bar{X}_{\alpha}^{(x)} \chi_{t}(x-\xi) d V(\xi) \wedge d \overline{z^{\alpha}} \wedge d{\overline{z^{\prime}}}^{I} \\
S_{t} \bar{\partial}_{M} \varphi(x) & =\sum_{|J|=q+1} \int \psi_{\bar{J}}(\xi) \chi_{t}(x-\xi) d V(\xi) \wedge d{\overline{z^{\prime}}}^{J} .
\end{aligned}
$$

Set $v=d \zeta^{1} \wedge \cdots \wedge d \zeta^{n-1} \wedge d \xi^{n}$. We may assume that $d V=d \overline{\zeta^{1}} \wedge \cdots \wedge d \overline{\zeta^{n-1}} \wedge \nu$. For each $J=\left(j_{1}, \ldots, j_{q+1}\right)$, there exist increasing indices $J^{*}=\left(j_{1}^{*}, \ldots, j_{n-2-q}^{*}\right)$ and $\epsilon^{J}= \pm 1$ such that $d \overline{\zeta^{1}} \wedge \cdots \wedge d \overline{\zeta^{n-1}}=\epsilon^{J} d{\overline{\zeta^{\prime}}}^{J} \wedge d{\overline{\zeta^{\prime}}}^{J^{*}}$. Assume that $\psi_{\bar{J}}$ are 
skew-symmetric. Thus

$$
\begin{aligned}
S_{t} \bar{\partial}_{M} \varphi(x) & =\sum_{|J|=q+1} \epsilon^{J} \int \psi_{\bar{J}}(\xi) \chi_{t}(x-\xi) d \bar{\zeta}^{J} \wedge d \bar{\zeta}^{\prime} J^{*} \wedge \nu \wedge d{\overline{z^{\prime}}}^{J} \\
& =\sum_{|J|=q+1} \frac{\epsilon^{J}}{(q+1) !} \int \bar{\partial}_{M}^{(\xi)} \varphi \wedge \chi_{t}(x-\xi) d{\overline{\zeta^{\prime}}}^{J^{*}} \wedge \nu \wedge d{\overline{z^{\prime}}}^{J}
\end{aligned}
$$

By Stokes' formula, (3.1) and (B.22), $S_{t} \bar{\partial}_{M} \varphi(x)$ equals

$$
\begin{aligned}
& \sum_{|J|=q+1} \frac{(-1)^{q+1} \epsilon^{J}}{(q+1) !} \int \varphi(\xi) \wedge a(\xi) \bar{\partial}_{M}^{(\xi)}\left(a^{-1}(\xi) \chi_{t}(x-\xi)\right) \wedge d \bar{\zeta}^{\prime} J^{*} \wedge v \wedge d{\overline{z^{\prime}}}^{J} \\
= & \sum_{|I|=q} \sum_{1 \leq \alpha<n} \sum_{|J|=q+1} \frac{-\epsilon^{J}}{(q+1) !} \int \varphi_{\bar{I}}(\xi) a(\xi) \bar{X}_{\alpha}^{(\xi)} \frac{\chi_{t}(x-\xi)}{a(\xi)} d \bar{\zeta}^{\alpha} \wedge d{\overline{\zeta^{\prime}}}^{I} \wedge d{\overline{\zeta^{\prime}}}^{J^{*}} \wedge v \wedge d{\overline{z^{\prime}}}^{J} \\
= & -\sum_{|I|=q} \sum_{1 \leq \alpha<n} \int \varphi_{\bar{I}}(\xi) a(\xi) \bar{X}_{\alpha}^{(\xi)}\left(a^{-1}(\xi) \chi_{t}(x-\xi)\right) d V(\xi) \wedge d \overline{z^{\alpha}} \wedge d \overline{\bar{z}^{\prime}} I
\end{aligned}
$$

Here the last identity is seen as follows. Set $d \bar{z}^{\alpha} \wedge d \bar{z}^{\prime I}=\epsilon_{\alpha I}^{J} d \bar{z}^{\prime J}$, if the identity can hold; otherwise, set $\epsilon_{\alpha I}^{J}=0$. In the above summation $\sum_{|I|=q} \sum_{|J|=q+1}$, we may restrict to terms with $\epsilon_{J}^{\alpha I} \neq 0$. For $\epsilon_{\alpha I}^{J} \neq 0$, we have

$$
\begin{aligned}
\epsilon^{J} d \bar{\zeta}^{\alpha} \wedge d \bar{\zeta}^{I} \wedge d{\overline{\zeta^{\prime}}}^{J^{*}} \wedge d \bar{z}^{\prime J} & =\epsilon^{J} \epsilon_{J}^{\alpha I} d \bar{\zeta}^{J} \wedge d{\overline{\zeta^{\prime}}}^{J^{*}} \wedge d \bar{z}^{\prime J} \\
& =\epsilon^{J} d{\overline{\zeta^{\prime}}}^{J} \wedge d{\overline{\zeta^{\prime}}}^{J^{*}} \wedge d \bar{z}^{\alpha} \wedge d \bar{z}^{I}
\end{aligned}
$$

Note that the last term is independent of $J$. We have $(q+1)$ ! such terms for a fixed $(\alpha I)$. Set $E_{\alpha}(\xi, x)=a(\xi) \bar{X}_{\alpha}^{(\xi)}\left(a^{-1}(\xi) \chi_{t}(x-\xi)\right)+\bar{X}_{\alpha}^{(x)}\left(\chi_{t}(x-\xi)\right)$. We get

$$
\left[\bar{\partial}_{M}, S_{t}\right] \varphi(x)=\sum_{|I|=q} \sum_{1 \leq \alpha<n} \int \varphi_{\bar{I}}(\xi) E_{\alpha}(\xi, x) d V(\xi) \wedge d \overline{z^{\alpha}} \wedge d{\overline{z^{\prime}}}^{I}
$$

which can be put into the form in the lemma.

We now derive the homotopy formula for the case b) via smoothing. We have

$$
\bar{\partial}_{M} S_{t} \varphi-\bar{\partial}_{M} \varphi=S_{t} \bar{\partial}_{M} \varphi-\bar{\partial}_{M} \varphi+\left[\bar{\partial}_{M}, S_{t}\right]\left(\varphi-S_{t^{\prime}} \varphi\right)+\left[\bar{\partial}_{M}, S_{t}\right] S_{t^{\prime}} \varphi .
$$

Fix $\rho<\rho_{1}<\rho_{0}$ and $\epsilon>0$. Fix $t^{\prime}>0$ sufficiently small such that $\left|S_{t^{\prime}} \varphi-\varphi\right|<\epsilon$ on $M_{\rho_{1}}$. By Lemma B.5, for all small $t$

$$
\sup _{M_{\rho}}\left|\left[\bar{\partial}_{M}, S_{t}\right]\left(\varphi-S_{t^{\prime}} \varphi\right)\right| \leq C \sup _{M_{\rho_{1}}}\left|\varphi-S_{t^{\prime}} \varphi\right| \leq C \epsilon .
$$


Let $S_{t^{\prime}} \varphi=\sum_{|I|=q} \psi_{\bar{I}} d{\overline{z^{\prime}}}^{I}$. Then $\left[\bar{\partial}_{M}, S_{t}\right]=\sum\left[b_{\bar{\alpha}} \partial_{x^{n}}, S_{t}\right]$ and $\left(\left[\bar{\partial}_{M}, S_{t}\right] \psi\right)(x)=\sum_{1 \leq \alpha<n} \sum_{|I|=q} \int \chi_{t}(y)\left(b_{\bar{\alpha}}(x)-b_{\bar{\alpha}}(x-y)\right) \partial_{x^{n}} \psi_{\bar{I}}(x-y) d V(y) \wedge d{\overline{z^{\prime}}}^{I}$.

This shows that $\lim _{t \rightarrow 0}\left[\bar{\partial}_{M}, S_{t}\right] S_{t^{\prime}} \varphi=0$ on $M_{\rho}$. We also have $\lim _{t \rightarrow 0} S_{t} \bar{\partial}_{M} \varphi=$ $\bar{\partial}_{M} \varphi$ on $M_{\rho}$. Therefore, (B.23) implies that $\lim _{t \rightarrow 0} \bar{\partial}_{M} S_{t} \varphi=\bar{\partial}_{M} \varphi$ on $M_{\rho}$. Now by case a), $\varphi_{t}=\bar{\partial}_{M} P_{M_{\rho}}^{\prime} \varphi_{t}+Q_{M_{\rho}}^{\prime} \bar{\partial}_{M} \varphi_{t}$ holds on $M_{\rho}$ as currents. Letting $t \rightarrow 0$ and then $\rho \rightarrow \rho_{0}$, we obtain $\varphi=\bar{\partial}_{M} P_{M_{0}}^{\prime} \varphi+Q_{M_{0}}^{\prime} \bar{\partial}_{M} \varphi$ on $M_{\rho_{0}}$ in the sense of currents.

\section{References}

[1] J. BRUNA and J. M. BURGUÉs, Holomorphic approximation and estimates for the $\bar{\partial}$ equation on strictly pseudoconvex nonsmooth domains, Duke Math. J. 55 (1987), 539-596.

[2] S.-C. Chen and M.-C. Shaw, "Partial Differential Equations in Several Complex Variables", AMS/IP Studies in Advanced Mathematics, Vol. 19, Amer. Math. Soc., Providence, RI, International Press, Boston, MA, 2001.

[3] G. B. Folland and E. M. STEIN, Estimates for the $\bar{\partial}_{b}$ complex and analysis on the Heisenberg group, Comm. Pure Appl. Math. 27 (1974), 429-522.

[4] K. O. FRIEDRICHS, The identity of weak and strong extensions of differential operators, Trans. Amer. Math. Soc. 55 (1944), 132-151

[5] X. Gong and S. M. WebsteR, Regularity for the CR vector bundle problem I, Pure Appl. Math. Q. 6 (2010), 983-998.

[6] X. Gong and S. M. WeBster, Regularity in the local CR embedding problem, J. Geom. Anal. DOI: $10.1007 / \mathrm{s} 12220$ - 010 - 9192 - 6.

[7] G. M. HENKIn, The Lewy equation and analysis on pseudoconvex domains, Russian Math. Surveys 32 (1977), 59-130.

[8] G. M. Henkin and A. V. Romanov, Exact Hölder estimates of the solutions of the $\bar{\partial}-$ equation, Izv. Akad. Nauk SSSR Ser. Mat. 35 (1971), 1171-1183.

[9] L. HöRMANDER, The boundary problems of physical geodesy, Arch. Ration. Mech. Anal. 62 (1976), 1-52.

[10] L. HöRMANDER, "An Introduction to Complex Analysis in Several Variables", North Holland, Amsterdam, 3rd edition, 1990.

[11] N. KERZMAN, Hölder and $L^{p}$ estimates for solutions of $\bar{\partial} u=f$ in strongly pseudoconvex domains, Comm. Pure Appl. Math. 24 (1971), 301-379.

[12] I. LIEB and R. M. RANGE, Lösungsoperatoren für den Cauchy-Riemann-Komplex mit $\mathcal{C}^{k}$ Abschätzungen, Math. Ann. 253 (1980), 145-164.

[13] L. MA and J. Michel, Local regularity for the tangential Cauchy-Riemann complex, J. Reine Angew. Math. 442 (1993), 63-90.

[14] L. MA and J. MiCHEL, Regularity of local embeddings of strictly pseudoconvex CR structures, J. Reine Angew. Math. 447 (1994), 147-164.

[15] J. MICHEL and L. MA, On the regularity of CR structures for almost $C R$ vector bundles, Math. Z. 218 (1995), 135-142.

[16] A. NAGEL and J.-P. RosAY, Nonexistence of homotopy formula for $(0,1)$ forms on hypersurfaces in $C^{3}$, Duke Math. J. 58 (1989), 823-827.

[17] C. Romero, "Potential Theory for the Kohn Laplacian on the Heisenberg Group", Thesis, University of Minnesota, 1991. 
[18] M.-C. SHAW, $L^{p}$ estimates for local solutions of $\bar{\partial}_{\mathrm{b}}$ on strongly pseudo-convex CR manifolds, Math. Ann. 288 (1990), 35-62.

[19] Y. T. SIU, The $\bar{\partial}$ problem with uniform bounds on derivatives, Math. Ann. 207 (1974), $163-176$.

[20] S. M. WebsteR, A new proof of the Newlander-Nirenberg theorem, Math. Z. 201 (1989), 303-316.

[21] S. M. Webster, On the local solution of the tangential Cauchy-Riemann equations, Ann. Inst. H. Poincaré Anal. Non Linéaire 6 (1989), 167-182.

[22] S. M. Webster, On the proof of Kuranishi's embedding theorem, Ann. Inst. H. Poincaré Anal. Non Linéaire 6 (1989), 183-207.

[23] S. M. WEBSTER, The integrability problem for CR vector bundles, In: "Several Complex Variables and Complex Geometry", Part 3 (Santa Cruz, CA, 1989), Proc. Sympos. Pure Math., 52, Part 3, Amer. Math. Soc., Providence, RI, 1991, 355-368.

Department of Mathematics University of Wisconsin Madison, WI 53706, USA gong@math.wisc.edu

Department of Mathematics University of Chicago Chicago, IL 60637, USA webster@math.uchicago.edu 